Florida International University FIU Digital Commons

$11-8-2012$

\title{
Silicon Photonic Device for Wavelength Sensing and Monitoring
}

German R. Vargas

Florida International University, gvarg008@fiu.edu

DOI: $10.25148 /$ etd.FI12111908

Follow this and additional works at: https://digitalcommons.fiu.edu/etd

\section{Recommended Citation}

Vargas, German R., "Silicon Photonic Device for Wavelength Sensing and Monitoring" (2012). FIU Electronic Theses and Dissertations. 734.

https://digitalcommons.fiu.edu/etd/734 


\section{FLORIDA INTERNATIONAL UNIVERSITY \\ Miami, Florida}

\section{SILICON PHOTONIC DEVICE FOR WAVELENGTH SENSING AND MONITORING}

A dissertation submitted in partial fulfillment of the requirements for the degree of DOCTOR OF PHILOSOPHY in ELECTRICAL ENGINEERING

by

Germán R Vargas López 
To: Dean Amir Mirmiran

College of Engineering and Computing

This dissertation, written by Germán R Vargas López, and entitled Silicon Photonic Device for Wavelength Sensing and Monitoring, having been approved in respect to style and intellectual content, is referred to you for judgment.

We have read this dissertation and recommend that it be approved.

Jean Andrian

Stavros Georgakopoulos

Jeffrey Fan

Arvind Agarwal

Roberto R Panepucci, Major Professor

Date of Defense: November 8, 2012

The dissertation of Germán R Vargas López is approved.

Dean Amir Mirmiran

College of Engineering and Computing

Dean Lakshmi N. Reddi

University Graduate School

Florida International University, 2012 


\section{(C) Copyright 2012 by Germán R Vargas López}

All rights reserved. 


\section{DEDICATION}

I dedicate this dissertation to my mother Nancy for her patient support during all these years, for teaching me about true love for a family and to persevere against all circumstances. To my brother Daniel for his encouragement. 


\section{ACKNOWLEDGMENTS}

I would like to thank all the people who made this work possible. My advisor Dr. Roberto R Panepucci who helped me with his guidance and assistance along my years at Florida International University and at CTI - Renato Archer. An especial consideration towards Prof. Jean Andrian who mentored me during my dissertation period and who had the time and patience during many conversations we had. I would also like to thank Dr. Stavros Georgakopoulos, Dr. Jeffrey Fan and Dr. Arvind Agarwal for being part of my committee and their collaboration during my dissertation research.

Along these years that I was working towards this dissertation, I had the privilege to have the help and support of wonderful people who share their time, expertise and friendship. I would like to thank William Fegadolli for his helpful collaboration, Dr. Bernardo Kyotoku for his hospitality at Campinas, Antonio Cesar Gozzi for his assistance during experiments at UNICAMP and Dr. Xuan Wang for useful conversations about optical silicon photonic chips, and collaboration in the initial stage of this research. I also want to express my gratitude to Maria Benincasa for her help regarding administrative and academic issues at FIU's ECE department. Experimental work in this research was possible thanks to the many staff members at CTI Renato Archer at Campinas. Specifically, I would like to thank Ms. Maria das Graças de Almeida for her assistance in CTI cleanroom and chip processing activities. Especially, I would like to thank Dr. Evandro Conforti at UNICAMP for allowing us to use his laboratory during our experiments.

This work was supported financially and institutionally by the Escuela Superior Politécnica del Litoral (ESPOL) under the direction of his Rector Dr. Moises Tácle. Also, during the initial doctoral years, the author was supported by FIU's College of Engineering Faculty Development Program. Finally, I want to acknowledge the financial support from CNPq during his visits to CTI Renato Archer at Campinas, Brazil. 


\author{
ABSTRACT OF THE DISSERTATION \\ SILICON PHOTONIC DEVICE FOR WAVELENGTH SENSING AND \\ MONITORING \\ by \\ Germán R Vargas López \\ Florida International University, 2012 \\ Miami, Florida \\ Professor Roberto R Panepucci, Major Professor
}

Over the last decade advances and innovations from Silicon Photonics technology were observed in the telecommunications and computing industries. This technology which employs Silicon as an optical medium, relies on current CMOS micro-electronics fabrication processes to enable medium scale integration of many nano-photonic devices to produce photonic integrated circuitry.

However, other fields of research such as optical sensor processing can benefit from silicon photonics technology, specially in sensors where the physical measurement is wavelength encoded.

In this research work, we present a design and application of a thermally tuned silicon photonic device as an optical sensor interrogator.

The main device is a micro-ring resonator filter of $10 \mu \mathrm{m}$ of diameter. A photonic design toolkit was developed based on open source software from the research community. With those tools it was possible to estimate the resonance and spectral characteristics of the filter. From the obtained design parameters, a 7.8 x $3.8 \mathrm{~mm}$ optical chip was fabricated using standard micro-photonics techniques. In order to tune a ring resonance, Nichrome micro-heaters were fabricated on top of the device. Some fabricated devices were systematically characterized and their tuning response were determined. From measurements, 
a ring resonator with a free-spectral-range of $18.4 \mathrm{~nm}$ and with a bandwidth of $0.14 \mathrm{~nm}$ was obtained. Using just $5 \mathrm{~mA}$ it was possible to tune the device resonance up to $3 \mathrm{~nm}$.

In order to apply our device as a sensor interrogator in this research, a model of wavelength estimation using time interval between peaks measurement technique was developed and simulations were carried out to assess its performance. To test the technique, an experiment using a Fiber Bragg grating optical sensor was set, and estimations of the wavelength shift of this sensor due to axial strains yield an error within $22 \mathrm{pm}$ compared to measurements from spectrum analyzer.

Results from this study implies that signals from FBG sensors can be processed with good accuracy using a micro-ring device with the advantage of ts compact size, scalability and versatility. Additionally, the system also has additional applications such as processing optical wavelength shifts from integrated photonic sensors and to be able to track resonances from laser sources. 


\section{TABLE OF CONTENTS}

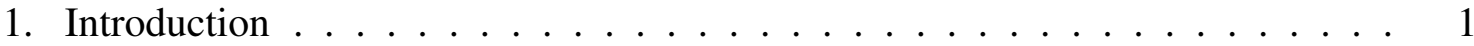

1.1 Optical Sensor Processing by Photonic Integrated Circuits . . . . . . . . . . 1

1.2 Silicon as an optical medium . . . . . . . . . . . . . . . . . . 2

1.3 Optical sensors . . . . . . . . . . . . . . . . . . 4

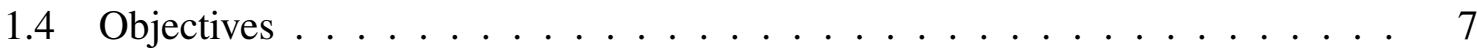

1.5 Organization of this dissertation $\ldots \ldots \ldots$. . . . . . . . 8

2. Theory of a Coupled Micro-ring Resonator Optical Filter . . . . . . . . . . . . 10

2.1 Mathematical modeling of micro-ring resonators in add-drop configuration . 10

2.2 Transfer Matrix Method . . . . . . . . . . . . . . . . . . . . 12

2.2.1 Performance Parameters of Micro-ring Resonators . . . . . . . . . . . . . . 17

2.3 Time Domain Analysis Method . . . . . . . . . . . . . . . . . . 23

2.3.1 Ring resonator parameter extraction using CMT model. . . . . . . . . . . . 30

3. Optical Sensors: Fiber Bragg gratings . . . . . . . . . . . . . . . . 34

3.1 Introduction . . . . . . . . . . . . . . . . . 34

3.2 Review of Fiber Bragg Grating Sensors . . . . . . . . . . . . . . . . 35

3.2.1 A spectral model of a Fiber Bragg Grating . . . . . . . . . . . . . . . . 37

3.2.2 Relation between strain and temperature measurands and the spectrum of

an $\mathrm{FBG} \ldots \ldots \ldots . \ldots . \ldots 39$

3.3 Fiber Bragg Grating sensor demodulation techniques . . . . . . . . . . . . . . . 42

3.3.1 FBG demodulation based on tunable optical filter . . . . . . . . . . . . . 44

3.3.2 Theoretical analysis of scanning and zero-crossing detection methods . . . 47

3.3.3 Towards a Wavelength Tracking Model . . . . . . . . . . . . . . 53

3.3.4 Wavelength center demodulation by time interval measurement method . . 55

4. Design of a Silicon Photonic Filter Device . . . . . . . . . . . . . . 57

4.1 Demodulation system characteristics / constraints / parameters . . . . . . . 57

4.2 Optical device design . . . . . . . . . . . . . . . . . . . . . . 62

4.2.1 Optical waveguide characteristics . . . . . . . . . . . . . 63

4.2.2 Micro-ring resonator radius . . . . . . . . . . . . . . . . . . 69

4.2.3 Resonances and free spectral range . . . . . . . . . . . . . . . . 72

4.2.4 Losses in a micro-ring resonator . . . . . . . . . . . . . . . . . . 74

4.2.5 Waveguide separation distance . . . . . . . . . . . . 75

5. Device Fabrication and Characterization . . . . . . . . . . . 81

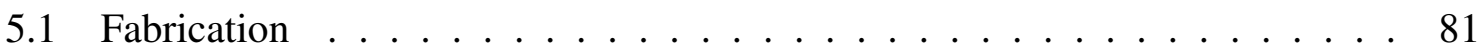

5.2 Optical characterization techniques . . . . . . . . . . . . 85

5.3 Single micro-ring resonator measurement results and analysis . . . . . . 88

5.4 Micro-ring resonator wavelength tuning by micro heater . . . . . . . . . . 93 
6. Wavelength to Time Interval Demodulation Employing a Tunable Silicon Microring Resonator . . . . . . . . . . . . . . . . . . . . . . . . . . . . 97

6.1 System Concept . . . . . . . . . . . . . . . . . . . . . . . . . . 98

6.1 .1 Time Interval Wavelength Demodulation _ . . . . . . . . . . . . . 100

6.2 System Simulation . . . . . . . . . . . . . . . . . . . . . . . . . . 103

6.3 Sensor interrogation experiments. . . . . . . . . . . . . . . . . 109

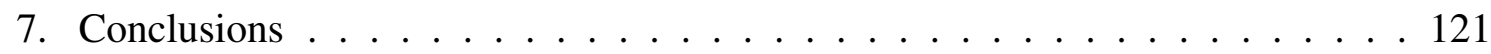

7.1 Future Work . . . . . . . . . . . . . . . . . . . . . . . . . 122

Bibliography . . . . . . . . . . . . . . . . . . . 124

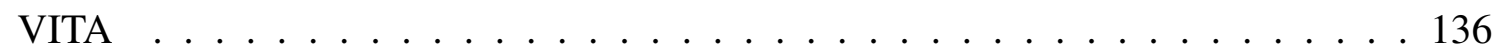




\section{LIST OF TABLES}

TABLE

PAGE

3.1 Comparison of FBG strain and temperature sensitivities found in literature. . 41

4.1 System components and power budget. . . . . . . . . . . . . . 61

4.2 Fitted parameters for effective index of a straight SOI waveguide (quasi-TE fundamental mode $). \ldots \ldots 68$

4.3 Fitting parameters for curvature function $f(R) \ldots \ldots \ldots 72$

5.1 Fabrication parameter design details for a SOI micro-ring resonator. . . . . . 83

5.2 Data extracted from Spectrum measurements for a single ring resonator device of $5 \mu \mathrm{m}$ radius (Quasi-TE mode). . . . . . . . . . . . . . . 89

5.3 Data extracted from Spectrum measurements for a single ring resonator device of $5 \mu \mathrm{m}$ radius (Quasi-TM mode). . . . . . . . . . . . . . . . 90

5.4 Parameters extracted from the quasi-TE mode spectrum response of a $5 \mu \mathrm{m}$ ring resonator. . . . . . . . . . . . . . . . . 91

6.1 Numerical simulation parameters of a MRR sensor interrogator. . . . . . . . 104 


\section{LIST OF FIGURES}

FIGURE

PAGE

$2.13 \mathrm{D}$ picture of a ring resonator in add-drop configuration. . . . . . . . . . . 11

2.2 Ring Resonator in add-drop configuration. . . . . . . . . . . . . . . . . 12

2.3 Chain matrix block diagram representation. . . . . . . . . . . . . . . 14

2.4 Calculated normalized intensities for the through and drop ports. Ring resonator radius $R=5 \mu m, n_{e f f} \approx 2.35$ near $1550 \mathrm{~nm}, \kappa^{2}=0.02$, and inner circulation factor of $0.992 . \ldots \ldots \ldots . \ldots$

2.5 Insertion $\operatorname{loss}\left(-10 \log \left(D_{\max }\right)\right)$ and Through port extinction $\left(-10 \log \left(T_{\min }\right)\right)$ for a symmetrically coupled ring resonator $R=5 \mu \mathrm{m} \ldots \ldots \ldots$. . . .

2.6 Simulation results of an asymmetrical ring resonator with $\kappa_{2}=0.1$ and inner circulation factor $a=1 . \ldots \ldots 20 . \ldots . \ldots 2 . \ldots 2 \ldots$

2.7 Add-drop ring resonator in Temporal Mode Coupling Analysis . . . . . . . . 25

2.8 Transmittance values for micro-ring parameter extraction based on the Coupling of Modes in Time method. . . . . . . . . . . . . 31

3.1 Fiber Bragg grating (FBG) sensor main characteristics and its operating prin-

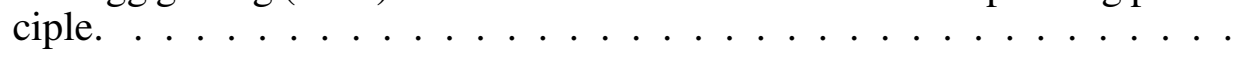

3.2 FBG Reflectance based on theoretical model from Eq. 3.3 (red color) and approximation using a Gaussian function (blue color). Grating length is $1 \mathrm{~cm}$, grating period is $0.545 \mu \mathrm{m}$, core effective index 1.42 and induced modulation index $\Delta n=1.5 e-4 \ldots \ldots . \ldots 38$

3.3 (a) Spectrum measurement using a narrow-band Fabry-Perot tunable filter. (b) Operating principle of the scanning Fabry-Perot spectrum measurement. 45

3.4 A tunable optical filter for FBG sensor demodulation in wavelength tracking operation. . . . . . . . . . . . . . . . 46

3.5 First derivative of the Optical Intensity signal obtained at the output of lockin amplifier. ...................... 52

3.6 Linear system model of a wavelength tracking system employing a tunable optical filter. . . . . . . . . . . . . . . . . 54

3.7 Principle of operation of the time-interval between minima (or maxima) measurement method. . . . . . . . . . . . 55

4.1 Wavelength Division Multiplexed sensor band allocation. The zoomed region corresponds to sensor channels covering the ITU-T C-band wavelength region. Adapted from [10] 
4.2 Material dispersion with Temperature dependence for $\mathrm{Si}$ (a) and $\mathrm{SiO} 2$ (b) . . 65

4.3 E-field mode profiles for quasi-TE (a) and quasi-TM (b) polarizations. . . . . 66

4.4 Effective index of a straight SOI waveguide (quasi-TE fundamental mode) of $450 \times 250 \mathrm{~nm}^{2}$ cross-section as a function of wavelength and temperature. 67

4.5 Equivalent index profile of a $2 \mu \mathrm{m}$ ring radius SOI channel waveguide of 450 $\mathrm{nm} \times 250 \mathrm{~nm}$ cross-section after applying conformal transformation. . . . 70

4.6 Curvature function $f(R)$ fit to numerical data of quasi-TE effective index of bent SOI waveguide. Inset represents the change in effective index as function of wavelength and temperature, colors represent same wavelength. 72

4.7 Resonance wavelengths of two MRR designs of radius $2 \mu \mathrm{m}$ and $5 \mu \mathrm{m}$ for quasi-TE and quasi-TM polarizations. . . . . . . . . . . . . 73

4.8 Upper view of a MRR geometric structure used for power coupling computation with 3D FDTD. . . . . . . ........... 76

4.9 Power cross-coupling coefficient $\kappa^{2}$ dispersion graph for $2 \mu m$ radius ring (a) and $5 \mu \mathrm{m}$ radius ring $(\mathrm{b})$, for quasi-TE mode. . . . . . . . . . . 77

4.10 Power cross-coupling coefficient $\kappa^{2}$ vs. bus-ring gap distance for $2 \mu \mathrm{m}$ radius ring (a) and $5 \mu \mathrm{m}$ radius ring (b), for quasi-TE and quasi-TM modes. . . . 78

4.11 Contour plot of Finesse (dotted color lines) and Maximum Drop port Efficiency $D_{\max }$ (color full lines) as function of power coupling efficiency and Field round trip loss. Orange colored patch represents a loss region for a $5 \mu \mathrm{m}$ radius ring design, the gray patch corresponds to a ring design of $2 \mu m$ radius. . . . . . . . . . . . . . . . . 79

5.1 CAD diagram of designed chip. . . . . . . . . . . . . . . . 82

5.2 Lithography cross-section. . . . . . . . . . . . . . . 83

5.3 Micrograph image of a single ring resonator with a micro heater on top. . . . 84

5.4 (a) Scanning Electron Microscopy (SEM) image of a device showing a break in a Nichrome micro heater. (b) Image of the same micro heater after'fixing' it using a Focused Ion Beam (FIB) machine that injected Pt material to close the break. . . . . . . . . . . . . . . . . . 84

5.5 Standard measurement setup for micro-ring resonator characterization. . . . 86

5.6 Schematic diagram for Optical characterization (spectrum measurement) of silicon photonic chips using an Optical Spectrum Analyzer (OSA). . . . 87

5.7 Alignment setup. . . . . . . . . . . . . . . . 88 
5.8 Quasi-TE spectrum response of a single micro-ring resonator of $5 \mu \mathrm{m}$ of radius and waveguide to ring gap distance of $200 \mathrm{~nm} . \ldots \ldots$. . . . .

5.9 Quasi-TM spectrum response of a single micro-ring resonator of $5 \mu \mathrm{m}$ of radius and waveguide to ring gap distance of $200 \mathrm{~nm} . \ldots \ldots$. . . . 90

5.10 Curve fit to data from quasi-TE spectrum measurement of a single microring resonator of $5 \mu \mathrm{m}$ of radius and waveguide to ring gap distance of $200 \mathrm{~nm} \ldots \ldots \ldots \ldots \ldots 2$

5.11 Test setup for tuning calibration. . . . . . . . . . . . . . . . . . . 93

5.12 Schematic diagram of the thermal tuning characterization of a micro-ring resonator filter. . . . . . . . . . . . . . . . . . . . . 94

5.13 Drop port transmission spectrum response after applying DC current (from 0 $\mathrm{mA}$ to $5 \mathrm{~mA}$ ) to a micro heater on top of the micro-ring resonator device. Color lines correspond to Lorentzian curve fit applied to data. . . . . . .

5.14 A micro-ring resonator resonance tuning response as a function of the square of DC current. . . . . . . . . . . . . . . . . . . . . . 95

6.1 Concept diagram of the wavelength detection system based on MRR device. A current source is applied to a micro-heater film on top of a Si MRR with a $\mathrm{SiO} 2$ cladding. A FBG sensor is employed to demonstrate the system capabilities to detect Bragg wavelength shifts due to strain or temperature changes in the sensor. Adapted from [72] . . . . . . . . . 99

6.2 Concept diagram of the time interval demodulation scheme based on MRR device (C)2011 IEEE. . . . . . . . . . . . . . . . . . . . . . . . 101

6.3 Simulation algorithm block diagram. . . . . . . . . . . . . . . . . 104

6.4 (a) Simulation results. The upper plot shows the MRR filter resonance tuning signal, the middle and lower plot shows the detected intensity signal after spectrum overlap of MRR filter transmittance and FBG reflectance at two wavelength shifts $(0.5$ and $2.5 \mathrm{~nm})$. (b) Results of time interval between peaks at 11 wavelength shifts after system simulation. The line corresponds to Eq. 6.5 (c)2011 IEEE. . . . . . . . . . . . . . . . . . 105

6.5 (a) Results of standard deviation (root mean squared error - RMSE) of detected wavelength as a function of SNR at different MRR total $Q$ values. FBG Bragg wavelength was simulated with a wavelength shift of $1.0 \mathrm{~nm}$ from a simulated MRR resonance of $1539.04 \mathrm{~nm}$. (b) Results of root mean squared error of detected wavelength as a function of an optical sensor full-width-at-half maximum (FWHM) (C2011 IEEE. . . . . . . . 108

6.6 Schematic diagram of time interval measurement setup using a laser source for calibration purposes. . . . . . . . . . . . . . . . 110 
6.7 (a) Oscilloscope traces of three laser lines after being scanned by a tunable ring resonator and selecting the TE mode response. The time interval in one period of the tuning (scan) triangular signal (shown in gray color) was measured by detecting the peaks of those traces. The "bumps" in between two peaks (near the center) represent the effect of residual TM mode, which can be reduced by adjusting a polarization controller. (b) Time interval measurement calibration curve for known laser wavelength values.

6.8 FBG strain calibration curve. The approximated strain sensitivity at 1552.821 nm was $0.758 \times 10^{-6} \mu \varepsilon^{-1} \ldots \ldots \ldots 113$

6.9 Schematic diagram of optical sensor characterization measurement. On left side, a setup is shown where axial strain was applied to a FBG sensor using a plastic cup and coins as weights. . . . . . . . . . . . 114

6.10 Oscilloscope traces showing interrogation of an strained FBG sensor. . . . . 116

6.11 (a) Comparison between time interval results from FBG interrogation and given by linear fit from OSA measurements. (b) The difference error from estimated wavelength shifts using time interval measurements and linear fit from OSA measurements. Blue line corresponds to estimations made with Maximum peak algorithm and Red line comes from Gaussian fit Algorithm. . . . . . . . . . . . . . . . . . . 117 


\section{CHAPTER 1}

\section{INTRODUCTION}

\subsection{Optical Sensor Processing by Photonic Integrated Circuits}

Silicon photonics have been an interesting and productive area of research over the last decade. This technology relies on current CMOS microelectronic materials and systems to be able to integrate many nanophotonic devices and build a photonic integrated circuit [1]. Many of the results in this field yielded new devices and components with designed functionality including waveguides, filters, electro-optical (E-O) controlled photonic modulators [2], thermo-controlled modulators and switches [3, 4]. However, the advancement of this field will also require research and development of integrated systems based on photonic devices, hopefully to address diverse applications.

Applying silicon photonics into other fields is a path followed by many researchers; one in particular is optical sensing. Sensors of this type have the advantage that they can be monitored remotely, tolerate harsh environments and have immunity to electromagnetic interference. However, one main issue in spectrally encoded optical sensing is monitoring or detection of the wavelength changes in the optical spectrum with high precision. Wavelength detection is also important in applications like bio-chemical spectroscopy, and optical Wavelength Division Multiplexing (WDM) networks.

Therefore, this study undertakes the wavelength monitoring issue through the design, fabrication and characterization of a silicon photonic device for optical sensing.

Innovations in optical sensors have advanced in such a way that today their applications are found in advanced products and services covering diverse market sectors. For example, optical sensing is essential for the development of smart systems in areas such as: homeland security, civil infrastructures and biomedical fields. Our proposed wave-

length monitoring system device can adapt its applicability into multiple scenarios, for 
example, it could be used for detection of dynamic changes in temperature/strain in optical sensors, closed loop control of the tuning and stability of optical fiber lasers [5], and also detecting wavelength shifts on ring resonator based sensors for label-free bio-sensing [6]. We envision that our system is an important step towards possibly achieving multipoint sensing where multiple sensor data are efficiently collected, processed and fed into databases which can be converted into relevant and useful physical information to the final user.

\subsection{Silicon as an optical medium}

Silicon is one of many materials used in the standard fabrication of integrated microelectronics systems. Recently, this material has become a suitable choice for integrated optics device fabrication due to its compatibility with existing micro-electronics manufacturing technology $[7,8,9]$. Silicon has three main factors that made it an interesting optical material. One is its transparency to infrared light, specifically in the range between $1.2 \mu \mathrm{m}$ to $1.7 \mu \mathrm{m}$. Many optical telecommunication components work in that infra-red region, therefore we can reuse those components and put them together with silicon photonics chip devices to build low-cost systems.

The second advantage of Silicon as an optical material is its high-refractive index compared to other materials such as silica ( $\mathrm{SiO} 2)$, silicon nitride (Si3N4) and some polymers. When a high-index core material is surrounded by a lower-index cladding layer then light can be confined inside its core due to total internal reflection. For example, the large-index contrast between a silicon core $(n=3.5)$ and silica $(n=1.5)$ cladding means that the effective wavelength of light inside the core is reduced, therefore a single mode optical field can be accommodated into a sub-micron sized waveguide. Another consequence of $\mathrm{Si} / \mathrm{SiO} 2$ high confinement is that waveguides with sharp bends are feasible. The overall 
benefit of employing silicon is that compact optical devices are possible to fabricate which in turn opens up the possibility of designing large-scale photonic integrated systems.

The third advantage is silicon's high thermo-optic coefficient $\left(\sim 10^{-4}{ }^{\circ} \mathrm{K}^{-1}\right)[10]$ which allow modifying the effective index of the optical mode propagating in a silicon waveguide. In optical resonant waveguide structures, the thermo-optic effect can be applied in order to tune an optical device resonance; e.g. optical switches and tunable filters using the thermo-optical effect can be demonstrated.

However, there are some challenges in the use of silicon as a material for monolithic integration of heterogeneous optical/photonic functions. One in particular is related to fabrication limitations. After fabrication, waveguides typically show wall roughness which produces light scattering and subsequently increases the propagation loss $[2,10]$. Although one benefit in using silicon for light confinement is due to its high index contrast, it also makes the optical structures highly sensitive to dimensional errors during fabrication. This is not considered a good thing because it reduces the tolerance in fabrication and makes it difficult to meet repeatable optical performance targets in designed devices [10]. Also, due to silicon high refractive index, coupling light into/out of a waveguide from an optical silica fiber is problematic because of the low coupling efficiency.

Additionally, silicon has low light emission efficiency. This means that it is not possible to design and fabricate light sources that compete with other materials such as those from III/V compound semiconductors, which are commonly found in LEDs and lasers [2]. However, researchers continue to work on solutions to integrate light emitting materials into CMOS platforms, with such material material integration problems already solved for the case of Germanium.

Despite the above-mentioned difficulties, recent research on silicon photonics has produced new ideas, techniques and devices that overcome those challenges. For example, Vlasov et al. achieved propagation losses of less than $3.6 \mathrm{~dB} / \mathrm{cm}$ in silicon waveguides 
[9]. Nanotapers solve the coupling between optical fibers and silicon photonic chips with low loss and wide bandwidth [11]. Those taper designs were widely used in Florida International University's (FIU) Nanophotonics group.

Research and development in silicon based opto-electronics is a driver today for future hybrid devices with applications ranging from telecommunications, large scale computing, sensor processing, imaging, display, energy conversion and lighting [12]. Some new companies born at the beginning of this century, such as Luxtera, employed silicon photonic technologies to build 4 × 10 Gbps active data transfer cables using integrated electro-optical systems in a chip. Also, this technology is actively developed by big technology players such as Intel and IBM. Therefore, using silicon as a material for E-O applications can have a huge impact in future technologies and it has the potential to be a pervasive technology in many interconnected devices.

\subsection{Optical sensors}

In this section, some definitions of an optical sensor and its subsystems are needed. Optical sensor refers a certain device that converts a physical measurand into a signal that produces a modulation of light in an optical system [13]. Those modulations can affect light transmission, dispersion or absorption among other effects.

Usually, an optical sensor is a part of an optical system that involves the sensor itself, a communication channel and a subsystem for signal generation, detection and processing. $[13]$

For sensing applications, any optical sensor can be classified in terms of: [14]

1. Physical magnitude to be measured.

Typical physical measurands are mechanical (strain, force, etc.), thermal (temperature), electromagnetic (electric voltage/current, magnetic fields), radiation (X-rays, 
nuclear radiation detected by luminescence or fluorescence) and chemical composition ( $\mathrm{pH}$, corrosion, etc.).

2. The spatial distribution of the measurement.

A distributed optical sensor system corresponds to a sensor that can measure a physical magnitude of interest along a line of space with a certain spatial resolution. A quasi-distributed optical sensor system allows measuring any physical variable at certain points in space along a single optical channel.

3. The nature of transduction.

An intrinsic optical sensor refers to an optical waveguide in which its internal transmission characteristics depend on the value to be measured.

An extrinsic optical sensor is a device where light interaction between the measurand and the optical waveguide occur outside the waveguide.

4. The manufacturing technology of the sensor device.

Optical sensors can be fabricated using fiber optic technology. Optical fibers are cylindrical waveguides made of silica. These devices are passive, have low losses at optical frequencies. Also, they show immunity to electromagnetic interferences, they are chemically and biologically inert and they are high temperature tolerant.

Also, optical sensors can be fabricated using integrated optics technology. This consists in optical waveguides integrated in a substrate chip with the advantage of miniaturization.

5. Modulation type of the optical sensor.

Optical sensors can be intensity modulated by a physical measurand. These types of sensors are very economical and simple to interrogate and process. 
Interferometric sensors are based on phase changes in light due to a physical variable. A coherent light source is required and they offer high measurement precision at increased cost.

Polarization based sensors utilize the modulation of light polarization. An example is the Faraday effect where electrical current can change the polarization in light through an optical material.

Wavelength encoded or spectral based sensors consist in the change of the light spectrum due to a change of the physical variable.

In this research, we are going to focus on a certain subset of optical sensors: intrinsic and wavelength-encoded. Those sensors depend on the modulation of the wavelength of light by an environmental variable. The most common wavelength-encoded sensor is the Fiber Bragg Grating, or FBG $[15,16]$. FBGs have the intrinsic capability to measure many physical parameters such as temperature, strain, pressure, chemical and biological variables [17]. Their use in many fields and industries is due to its quasidistributed sensing capabilities and relative low cost [18]. These fiber gratings because of their wavelength-encoded nature, are independent to fluctuations in light intensity which make them resilient to connector and fiber losses. Additional advantages of these sensors are their passive nature, which means that no external power is required, small size, electromagnetic interference immunity and their low loss at optical C-band (1550 nm), which made them good candidates for remote sensing applications.

It is worth mentioning that wavelength-encoded or spectral sensors can be technologically integrated in order to further reduce their size and enable low cost and ultra-compact devices, which can be useful in biological applications such as protein detection and DNA analysis for medical diagnostics [19]. 
Both types of spectral-encoded sensors (FBG and integrated) need a detection system that demodulates or extract a measurand parameter (e.g. temperature, strain or other) from the wavelength of light spectrum. The most common method to detect changes in the spectrum wavelength is based on a special measurement apparatus known as monochromator, or optical spectrum analyzer. Those analyzers have large sizes and are very expensive, which increase the overall cost of any optical sensor system.

\subsection{Objectives}

The proposed study will focus on the main shortcomings of other wavelength detection/monitoring methods, namely in terms of size, resolution and dynamic response. In summary, our aim will be to be able to design, implement and characterize a micro-optical device to offer:

1. Highly integrated platform to process an array of sensors.

2. Better resolution compared to mechanically tuned alternatives.

3. Adequate readout speed.

4. Low sensitivity to external influences.

Our research will undertake the problem of monitoring the wavelength shift of a perturbed Fiber Bragg grating due to external influences (strain, temperature). This problem was selected because of the wide application scenarios of optical sensors in civil engineering structures to prevent damages in infrastructures.

Based on this context, we devise a solution for the wavelength sensing problem by following an approach based on using a ring resonator as a tunable resonant filter to scan the FBG reflectance response due to changes such as strain or temperature variations. [20] We propose an approach consisting in applying an interrogation scheme in such a way 
that one port resonance response of the ring resonator structure would match the FBG reflectance at a certain wavelength. Then, whenever the FBG is perturbed by strain or temperature, its wavelength shift will be detected by applying a signal processing method based on Mora et al. [21]. With this signal processing method, any wavelength shift from a sensor response is translated into a time interval measurement. In summary, our approach will use a ring resonator as a spectral sliding window element by thermo-optically sweeping its resonance, and measure the time interval between resonance intensity peaks to infer wavelength shifts.

\subsection{Organization of this dissertation}

The overall organization of this study is formed of six chapters, including this introduction. Chapter 2 begins with two theoretical models known as the Transfer Matrix method and the Coupling of Modes in Time. Both models give a foundation for the analysis of an optical device, such as a silicon micro-ring resonator.

In chapter 3, a review of FBG sensors is covered. This chapter is concerned with how those sensors work, and the methods used to extract physical measurands by demodulating the wavelength shifts of those type of sensors. Also, this chapter presents common techniques to characterize the sensitivity of those sensors due to temperature and strain.

In chapter 4, a detailed system design and device design are explained. System design is needed in order to connect the FBG sensor requirements with the proposed photonic device. System constraints are introduced to lay out a basic framework on the operation of the demodulation system. A micro-ring resonator device design will be explained. By a thorough optical design based on numerical EM techniques jointly with some numerical model fits, approximations of the micro-ring resonator parameters are obtained. Also, from those models, basic micro-ring waveguide design parameters can be derived, which were applied for fabrication. 
Chapter 5 presents a brief explanation of the fabrication of an optical chip with some micro-ring resonators. Next, the chapter describes two optical and thermal tuning characterization methods. Some explanations of the results from those tests are given. That information helps us to apply our ring resonator filter device in a wavelength demodulation system.

Chapter 6 presents a demonstration of a wavelength shift demodulation technique using a tunable micro-ring resonator filter, which applies a time interval peak-to-peak measurement. An explanation on how the system works is presented together with a basic system model. A system simulation based on that model is implemented to show its operation and to determine the minimum wavelength shift that can be detected under noise conditions. Following the system description, experiments are described by first obtaining a calibration of the micro-ring resonator wavelength shift demodulation system employing a laser source as a known wavelength reference. After that, the application of this system is tested on a FBG sensor subjected to an axial strain. Two data processing techniques (maximum peak search and Gaussian fit) were applied to the data extracted from this demodulation system. By employing a Gaussian fit, accuracy of the wavelength shift detection was improved compared to maximum peak search data processing.

Finally, this dissertation concludes in Chapter 7 with a brief summary and implications of the findings for future research. 


\section{CHAPTER 2}

\section{THEORY OF A COUPLED MICRO-RING RESONATOR OPTICAL FILTER}

A particular integrated photonic device, which is the main focus of this study is the

micro-ring resonator. A micro-ring resonator is a special type of optical cavity that has multiple resonance wavelengths in its spectral response. Its basic functionality resembles other types of resonators, such as the Fabry-Perot etalon. Its main advantage is its easy integration with other components while reducing the overall footprint of a photonic integrated circuit.

A micro-ring resonator is one of the basic components available in the photonic design toolkit. Since the late 90s until recent times, grown interest about this device has been found in the literature. Evidence of that is found in many applications, such as optical filter/switches [22], add-drop multiplexers [23], optical modulators [2, 24] and optical sensors [6]. The importance of studying a micro-ring resonator lies in its potential use as a sensor interrogation system.

In this chapter, a theoretical analysis of this device is presented. Two theoretical approaches are applied to a micro-ring resonator analysis. The first is the Transfer Matrix method, which was a common methodology employed in the analysis of fiber ring resonator systems [25]. Another method known as coupling of modes in time is a technique coming from microwave theory. This time domain technique will prove its usefulness later on in the experimental characterization of micro-ring resonator filter structures.

\subsection{Mathematical modeling of micro-ring resonators in add-drop configuration}

In this section, a theoretical description of a ring resonator operation is described. A device in add-drop configuration was chosen because of its application as a wavelength selective filter or channel selector commonly found in telecommunications applications. 


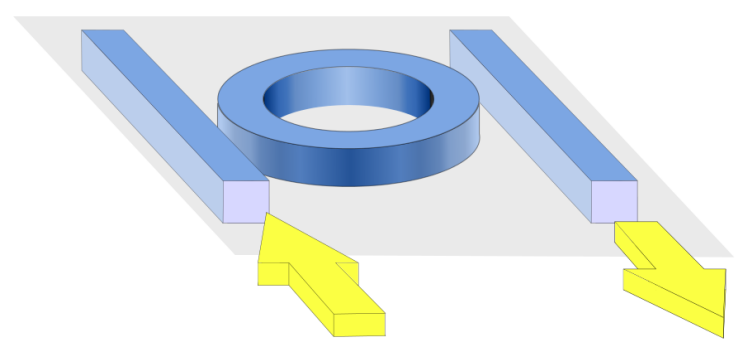

Figure 2.1: 3D picture of a ring resonator in add-drop configuration.

But more importantly, in this research, this device is going to be employed as a spectrum interrogator.

A picture of a ring resonator in add-drop configuration is shown in Fig. 2.1. Light propagates through a dielectric waveguide known as bus waveguide. This waveguide is needed to couple energy into a ring waveguide. What occurs when we inject light into this structure is that light recirculates inside the ring and light-wave resonances appear. This happens because a fraction of the energy transported on the bus waveguide is coupled into the ring, but as more energy is transported through the main waveguide, multiple coupling occurs. Those couplings generate clock-wise waves inside the ring, which add destructively or constructively based on their respective phases as they travel around. Therefore, to reach resonance, a phase condition of $\theta=k n_{e f f} L=m 2 \pi$ must be met. Here, $k$ is the wave-number $\left(k=\frac{2 \pi}{\lambda}\right), n_{e f f}$ is the real part of effective index of the optical eigenmode and $L$ is the circumference of the ring. Taking into account this condition, we can obtain the resonance wavelength:

$$
\lambda_{m}=\frac{n_{e f f} \cdot L}{m}
$$

Therefore, there are multiple resonances in an optical ring structure, and they depend on the ring dimensions (radius), and waveguide structure (effective index). Those resonances are separated from each other and their wavelength difference is known as the free-spectral-range or FSR. The amount of separation is dependent on the radius and ef- 


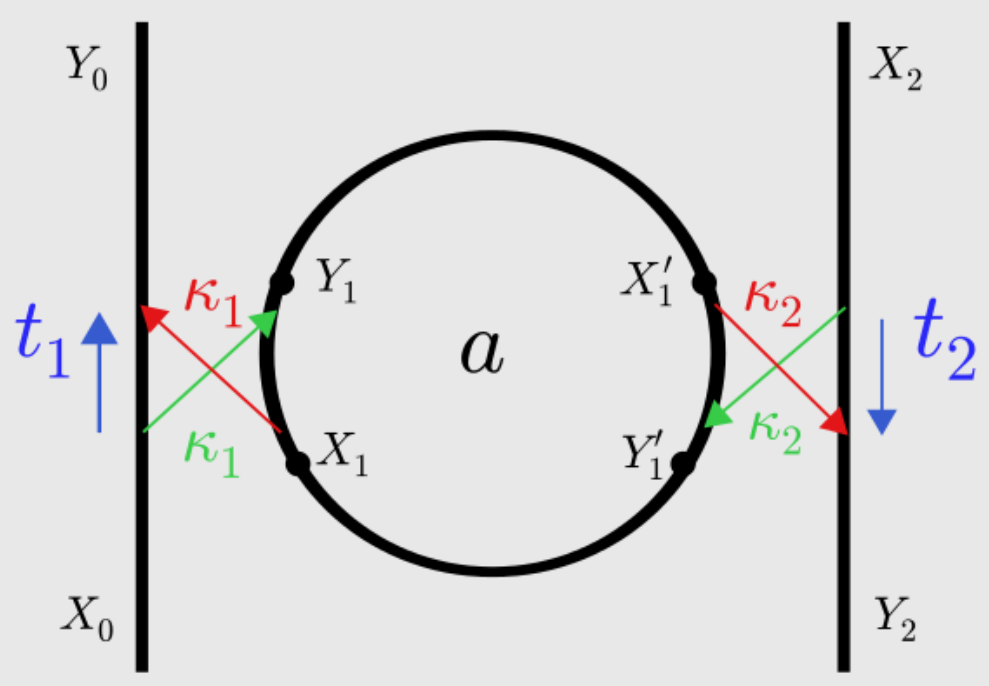

Figure 2.2: Ring Resonator in add-drop configuration.

fective index. In the next section, a theoretical analysis is made to obtain the spectrum response of this resonator and its main parameters.

\subsection{Transfer Matrix Method}

A diagram of a ring resonator in add-drop configuration is shown in Fig. 2.2. This adddrop device configuration can be decomposed into two important parts: a coupler region and a ring waveguide. The ring has a radius $(R)$ and an inner circulation factor $(a)$. If the ring has no internal loss then $a=1$. The ring resonator has two couplers, which allow a fraction of energy to be transferred in and out the straight waveguides. In our analysis, we introduce the following notation for cross-coupling field coefficients as $\kappa_{1}$ and $\kappa_{2}$ and straight-through coefficients $t_{1}$ and $t_{2}$, as they can be seen in Fig. 2.2.

Some assumptions are made for simplification purposes. First, this analysis considers the Electric field (E-field) only and assumes the waveguides are single-mode. Second, the field is propagating in one direction, there is no backward propagating fields or reflections 
in the system. Third, energy coupling between the bus waveguide and ring is lossless. The last one also known as the unitary condition, comes from energy conservation principles:

$$
\left|\kappa_{1}\right|^{2}+\left|t_{1}\right|^{2}=\left|\kappa_{2}\right|^{2}+\left|t_{2}\right|^{2}=1
$$

The first coupler field interaction is shown in Fig.2.2 and it can be described by a scattering or S-matrix [26, 27], and defined as:

$$
\left(\begin{array}{c}
X_{0} \\
X_{1}
\end{array}\right)=\left(\begin{array}{ll}
t_{1} & j \kappa_{1} \\
j \kappa_{1} & t_{1}
\end{array}\right)\left(\begin{array}{c}
Y_{0} \\
Y_{1}
\end{array}\right)
$$

where $X_{0}, X_{1}$ and $Y_{0}, Y_{1}$ are the input and output E-field amplitudes shown in Fig. 2.2. As light enters inside the ring, $Y_{1}$ field's phase accumulates and its amplitude decreases due to internal loss. This phase shift $\theta$ is proportional to the propagation constant $\beta$ and the circumference of the ring $L=2 \pi R$. The propagation constant is defined as $\beta=\frac{2 \pi n_{g}}{\lambda}$, where $n_{g}=\frac{\partial \beta}{\partial k_{0}}$ is known as the "group index". For simplification purposes, we choose instead of the "group index" the "effective refractive index" of a waveguide denoted as $n_{\text {eff }}$. Therefore the overall optical phase shift is:

$$
\theta=\frac{2 \pi n_{e f f}}{\lambda} \cdot L
$$

When the field $Y_{1}$ travels inside the ring it will reach the lower coupler shown in Fig.2.2 and the field $X_{1}^{\prime}$ at the second coupler is:

$$
X_{1}^{\prime}=\sqrt{a} \cdot \exp \left(j \frac{\theta}{2}\right) Y_{1}
$$

where $a$ accounts for the loss inside the ring, and the phase accumulation corresponds to half-way circle propagation of the field.

The ring waveguide input and output field equations can also be represented as a scattering matrix directly. Here we use a variable $z=\sqrt{(a \cdot \exp (j \theta))}$, where $\sqrt{a}$ is the loss 
(or gain in special circumstances) when the field propagates half circle. The relationship of the fields inside the ring becomes:

$$
\left(\begin{array}{l}
X_{1}^{\prime} \\
X_{1}
\end{array}\right)=\left(\begin{array}{ll}
0 & z \\
z & 0
\end{array}\right)\left(\begin{array}{l}
Y_{1}^{\prime} \\
Y_{1}
\end{array}\right)
$$

The second coupler input and output E-field relation can be expressed as an S-matrix:

$$
\left(\begin{array}{l}
Y_{2} \\
Y_{1}^{\prime}
\end{array}\right)=\left(\begin{array}{ll}
t_{2} & j \kappa_{2} \\
j \kappa_{2} & t_{2}
\end{array}\right)\left(\begin{array}{l}
X_{2} \\
X_{1}^{\prime}
\end{array}\right)
$$

While scattering matrices are easy to derive from the field coupling relations, one problem is that those relations cannot help when the optical components are connected in cascade to reconstruct the whole system. A solution is to convert the S-matrices to Chain or Transmission matrices [28].

A cascade system analysis [25] consists on representing the input and output E-fields at each coupler and ring sub-systems by chain or transmission matrices connected in series to obtain an overall transfer function. A block diagram of the process is shown in Fig. 2.3.

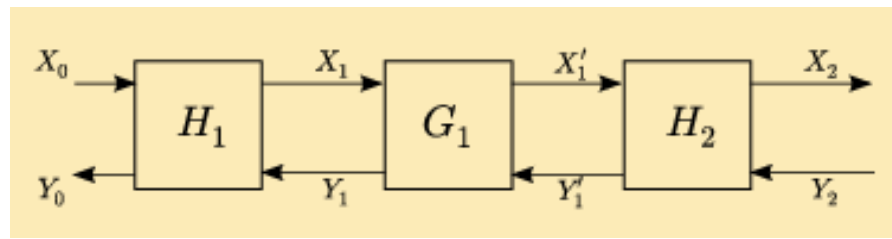

Figure 2.3: Chain matrix block diagram representation.

We can obtain the total matrix representation as the product of each matrix starting by the last coupler, in other words:

$$
H_{T}=H_{2} \cdot H_{R} \cdot H_{1}
$$


The final step is to solve the field amplitudes in terms of the overall ABCD matrix representation, Eq. 2.9 below shows the relationship between the drop port $Y_{2}$ and through port $Y_{0}$ outputs in terms of the input field $X_{0}$ and $X_{2}$.

$$
\left(\begin{array}{l}
X_{2} \\
Y_{2}
\end{array}\right)=\left(\begin{array}{ll}
A & B \\
C & D
\end{array}\right)\left(\begin{array}{l}
X_{0} \\
Y_{0}
\end{array}\right)
$$

The input field $X_{2}$ is known as the "add input port". To simplify, we can assume there is no signal at the add port, thus $X_{2}=0$. From Eq.2.9, the through port modal field amplitude ratio $(t)$ is expressed as:

$$
t=\frac{Y_{0}}{X_{0}}=-\frac{A}{B}
$$

And the drop port to input ratio denoted as $d$ is expressed as:

$$
d=\frac{Y_{2}}{X_{0}}=C-D \cdot \frac{A}{B}
$$

The amplitude ratios $t$ and $d$ completely characterize the transfer function of the ring resonator. By solving the matrix relation of the overall cascaded system described in Eq. 2.9, we obtain the following mode amplitude ratios:

$$
t=\frac{Y_{0}}{X_{0}}=\frac{t_{2} z^{2}-t_{1}}{t_{1} t_{2} z^{2}-1}
$$

And,

$$
d=\frac{Y_{2}}{X_{0}}=\frac{\kappa_{1} \kappa_{2} z}{t_{1} t_{2} z^{2}-1}
$$


The expressions 2.12 and 2.13 represent complex valued and normalized mode field amplitudes. By multiplying their respective complex conjugates and recalling that $z=$ $a^{\frac{1}{2}} \cdot e^{-j \frac{\theta}{2}}$, we obtain the relative intensities or output power at the through $T$ and drop $D$ ports [29] [30].

$$
T=\frac{t_{1}^{2}-2 t_{1} t_{2} a \cos \theta+t_{2}^{2} a^{2}}{1-2 t_{1} t_{2} a \cos \theta+t_{1}^{2} t_{2}^{2} a^{2}}
$$

And,

$$
D=\frac{\kappa_{1}^{2} \kappa_{2}^{2} a}{1-2 t_{1} t_{2} a \cos \theta+t_{1}^{2} t_{2}^{2} a^{2}}
$$

The response of both the through and drop output ports were calculated and results are shown in Fig. 2.4. Calculations of normalized intensity were done for a ring resonator with a radius of $5 \mu \mathrm{m}$, symmetric cross-coupling field coefficients $(\kappa=0.14)$ and effective index $\left(n_{e f f} \approx 2.35\right.$ at $\left.1550 \mathrm{~nm}\right)$. In this calculation we applied typical values of internal loss of $0.8 \%$, which corresponds to an inner circulation factor of 0.992 as reported in the literature [31]. The spectrum at the through output port has a response similar to a "comb" filter with notches at resonance wavelengths. The drop port response (shown in red color) has peaks located at the resonances of the device. The through port "notches" do not reach zero because the device is not critically coupled. This means that the fields inside the ring do not couple with enough strength to have destructive interference with the transmitted port fields when the device is on-resonance [29].

The drop port spectrum has peaks that do not reach unitary magnitude, so there is no full power transfer from the input port to the drop output port. To have full power transfer, negligible internal losses are required as well as asymmetric coupling. Silicon ring resonators have low propagation losses, e.g. $3.6-3.7 \mathrm{~dB} / \mathrm{cm}$ at $1.55 \mu \mathrm{m}$ wavelengths [9], but there are other loss sources that deteriorate the performance of a ring resonator. Therefore a scenario of incomplete power transfer is often found in fabricated devices. 

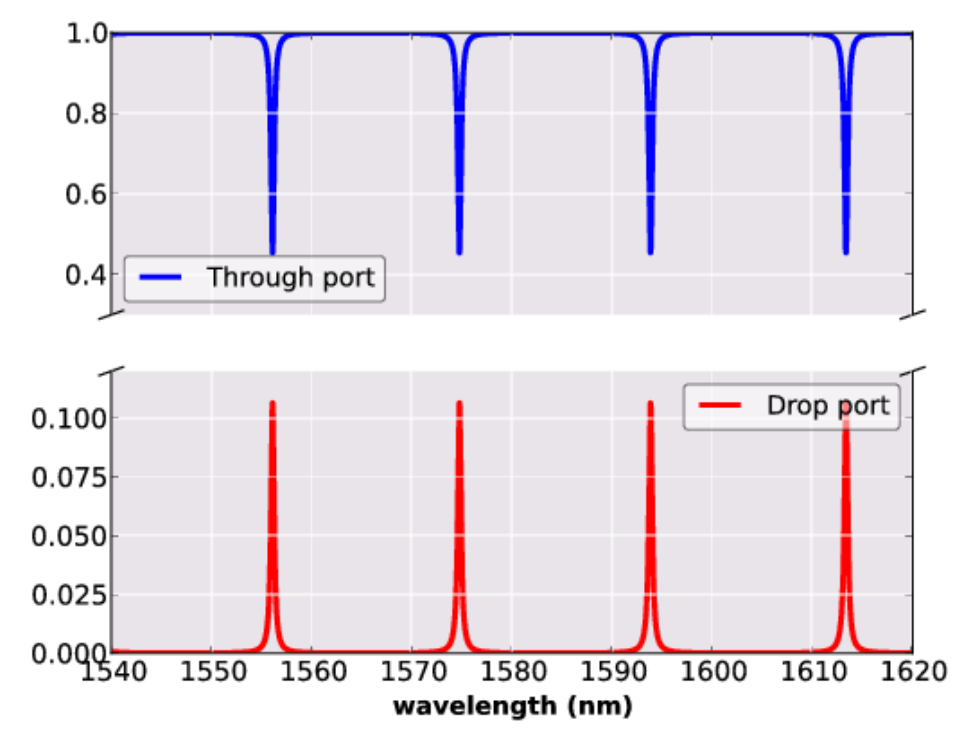

Figure 2.4: Calculated normalized intensities for the through and drop ports. Ring resonator radius $R=5 \mu \mathrm{m}, n_{e f f} \approx 2.35$ near $1550 \mathrm{~nm}, \kappa^{2}=0.02$, and inner circulation factor of 0.992 .

In the next section, more details about ring resonator performance parameters are given. Those metrics will prove useful to characterize devices and to evaluate device operations in many applications.

\subsubsection{Performance Parameters of Micro-ring Resonators}

A micro-ring resonator's performance is characterized by some figures of merit. Those parameters can be deduced from the results obtained in the previous section, additionally, those figures can be extracted by spectral characterization measurements.

When the ring is on resonance, a set of parameters characterize its performance. One of them is the Maximum drop port Efficiency, which is defined as $D_{\max }=\max \{D\}$, and is expressed as:

$$
D_{\max }=\frac{\kappa_{1}^{2} \kappa_{2}^{2} a}{\left(1-t_{1} t_{2} a\right)^{2}}
$$


The drop port efficiency in Eq. 2.16 is also expressed in logarithmic form, this is known as the Insertion Loss. A second parameter extracted when the ring is on-resonance is the Through port Extinction, which measures how much attenuation occurs when the ring is on-resonance. The amount of extinction loss shows how efficient is the power transfer from the input port to the drop port [29]. The through port extinction is $T_{\min }=$ $\min \{T\}$, which yields:

$$
T_{\text {min }}=\frac{\left(t_{1}-t_{2} a\right)^{2}}{\left(1-t_{1} t_{2} a\right)^{2}}
$$

Fig. 2.5 shows simulated examples of the insertion loss and port extinction of a ring resonator with radius $(R=5 \mu \mathrm{m})$ at three values of cross-coupling power coefficients $\left(\kappa^{2}=0.1,0.05,0.01\right)$. The independent variable is the inner circulation factor as a measure of how lossy the ring resonator is. In this calculation, we assumed that both couplers in the ring are symmetric. As Fig. 2.5(a) shows, the insertion loss only reaches $0 \mathrm{~dB}$ when there is no internal losses in the ring $(a=1)$. Although silicon waveguides have low loss in the infra-red region, this situation is very unlikely to happen in practical passive devices. As the bus to ring coupling $\left(\kappa^{2}\right)$ is reduced, less power is transferred to the drop port, increasing the insertion loss. This loss is further exacerbated as the internal circulator factor diminish due to increasing propagation loss.

In Fig. 2.5(b) we observe that the through port extinction is not high, even when there is no internal loss ( $T_{\min }$ do not exactly reach the zero value). Increasing the bus to ring coupling makes the through port attenuation (extinction) higher. The "critical coupling" condition is reached when $T_{\min }=0$, and it only occurs if the add-drop ring resonator has asymmetric coupling [29].

The drop port efficiency and through port extinction transfer functions were obtained from a simulated asymmetric coupled ring resonator with a inner circulation factor ( $a=$ 0.99). Fig. 2.6 show the simulated results when the second coupler is fixed with a value 
(a)

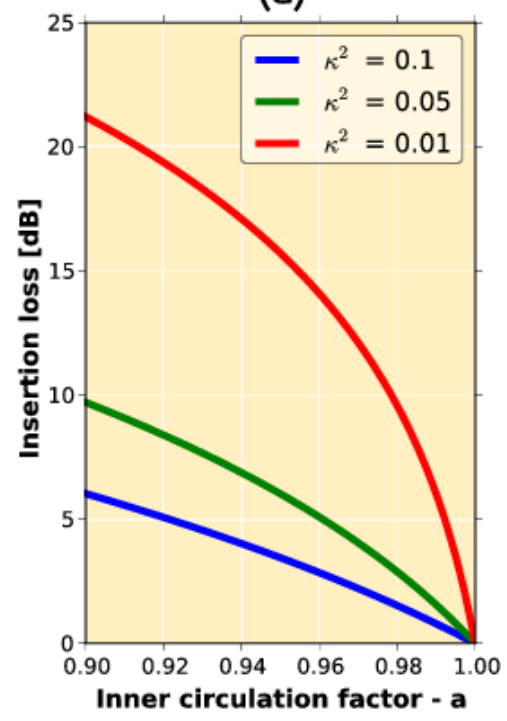

(b)

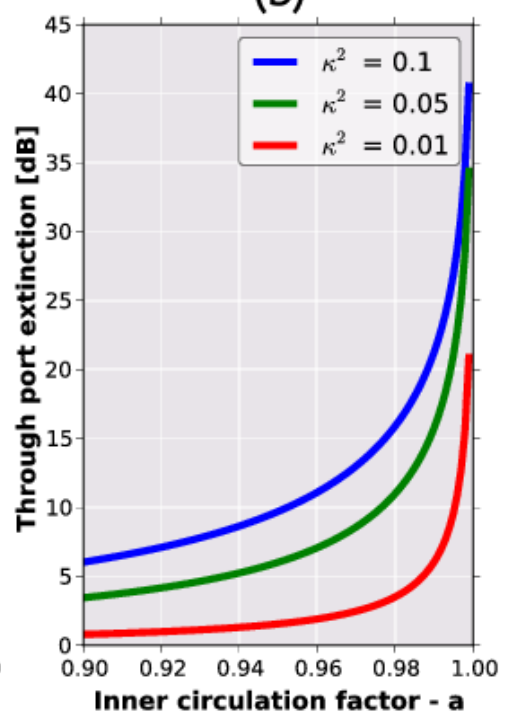

Figure 2.5: Insertion $\operatorname{loss}\left(-10 \log \left(D_{\max }\right)\right)$ and Through port extinction $\left(-10 \log \left(T_{\min }\right)\right)$ for a symmetrically coupled ring resonator $R=5 \mu \mathrm{m}$.

$\kappa_{2}=0.1$, and the independent variable is the first coupler $t_{1}=\sqrt{1-\kappa_{1}^{2}}$. When $t_{1}=1$ there is no coupling to the ring resonator, and all light goes to the main waveguide on the through port. However, there is a point when the extinction is zero, which also yields a maximum drop efficiency (less insertion loss), this point corresponds to the "critical coupling" situation and it only occurs when the internal loss $(a)$ is equal to the ratio of the two couplers $\left(\frac{t_{1}}{t_{2}}\right)$.

We can recall from Fig. 2.4 that the spectrum response has periodic notches (through port) or peaks (drop port) at distinct resonances. Another important figure of merit in ring resonators is the free-spectral-range $(F S R)$, which is the separation between adjacent resonance wavelengths.

In a simplified model, we can assign to each resonance an integer mode number $m=$ $\frac{n_{e f f} 2 \pi R}{\lambda_{m}}$. The FSR is obtained by solving the difference $\lambda_{m+1}-\lambda_{m}$. However, because a ring resonator has waveguide dispersion, the group index $n_{g}=\frac{\partial \beta}{\partial k_{0}}$ is a device parameter that usually is not constant over a large range of wavelengths. This situation produces 


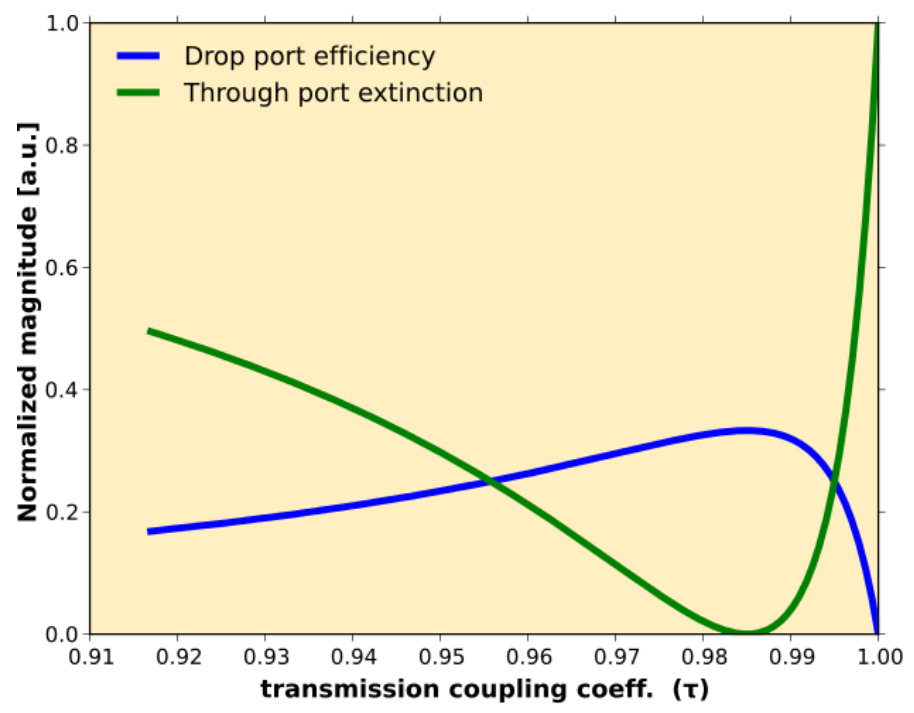

Figure 2.6: Simulation results of an asymmetrical ring resonator with $\kappa_{2}=0.1$ and inner circulation factor $a=1$.

an uneven separation between resonances. To account for this, we have to use the group index instead of the effective refractive index to determine the free-spectral-range. The amount of FSR can be expressed quantitatively as: [32]

$$
F S R \approx \frac{\lambda_{m}^{2}}{n_{g} 2 \pi R}
$$

Therefore, the amount of dispersion present in the waveguide and the ring radius control the FSR of the resonator. As we reduce the size of a ring resonator, its resonance separation is increased. For example, this issue has been proven useful in telecommunications, specifically, in Wavelength Division Multiplexing (WDM) applications. In such systems a large separation between resonances is required to accommodate many channels within a spectral band $[3,8,33]$.

A third important parameter characterizes the line-shape of the resonance. This parameter measures the $3 \mathrm{~dB}$ bandwidth response of the ring resonator transfer function, and it is known as the half-width-at-half-maximum or full-width at half-maximum (FWHM). 
A complete derivation of the FWHM based on the transfer matrix method is outside the scope of this analysis, and it can be found in some references [34, 35].

A ring resonator with different coupling characteristics at the two waveguides has a line-width that can be expressed as:

$$
\delta \lambda=\frac{\lambda_{m}^{2}}{\pi n_{g} L} \arccos \left[\frac{1+\left(t_{1} t_{2} a\right)^{2}-4 t_{1} t_{2} a}{-2 t_{1} t_{2}}\right] \approx \frac{\lambda_{m}^{2}}{\pi n_{g} L} \cdot \frac{1-a t_{1} t_{2}}{\sqrt{a t_{1} t_{2}}}
$$

We can reduce Eq. 2.19 complexity by assuming a ring resonator with very similar couplers $\left(t_{1} \approx t_{2}\right)$, weak cross-coupling $(\kappa<<1)$ and negligible internal loss $(a \approx 1)$. Therefore, Eq. 2.19 becomes:

$$
\delta \lambda=\frac{\lambda_{m}^{2}}{\pi n_{g} L} \cdot \frac{1-t^{2}}{t}
$$

A ring resonator spectral response is similar to a Fabry-Perot etalon [36], an often quoted parameter in etalon devices is the Finesse $(\mathscr{F})$. This parameter is independent of any geometrical dimensions and refractive index, and is defined as a ratio of the FSR and FWHM. From Eq. 2.18 and 2.20 we have:

$$
\mathscr{F}=\pi \cdot \frac{t}{1-t^{2}}
$$

The finesse is a figure of merit that depends on the internal losses and the coupling losses in the resonator. Higher finesse normally is accompanied with a sharper resonance and large resonance separation.

A measure of filter selectivity is often characterized by the Quality $(Q)$ factor. This is an essential parameter in resonators because of two reasons: first it is a way to assess the amount of loss in the resonator and second the $Q$-factor is related to the sharpness of a resonance. Highly selective filters thus have narrow line-widths or high Quality factors. In this research those filters can be employed as spectrum interrogators due to their narrow 
line-widths. On the other hand, in telecommunications applications, it may be necessary to have wide bandwidth to allow transport of broadband channels. Therefore, low $Q$ factor is suitable in such cases, but one problem is that single ring resonators have high crosstalk due to low extinction ratios. In those cases, more complex, higher order filters using multiple rings can be used.

In general, the Quality factor is defined as the time averaged stored energy per cycle divided by the energy lost in an optical cycle [23].

$$
Q=\frac{\omega_{0} \cdot \text { energy stored in resonator }}{\text { energy dissipated by resonator }}
$$

There are three $Q$ factors to consider in a resonator. One is the intrinsic or "unloaded" $Q$ factor associated to internal losses only. The second is the external $Q$ factor, which is related to losses due to external coupling only. And finally, the "loaded" $Q$ factor that accounts for internal and external losses [30]. The "loaded" $Q$ factor can be extracted from spectral measurements and is approximated as: [29, 23]

$$
Q \approx \frac{\lambda_{m}}{\delta \lambda} \approx \frac{f_{m}}{\delta f}
$$

where, $\lambda_{m}$ is the m-th order resonance, $\delta \lambda$ is the FWHM. If the ring resonator is measured in terms of optical frequencies, the $Q$ factor is the ratio between the resonance frequency $f_{m}$ and $3 \mathrm{~dB}$ bandwidth $\delta f$. We can derive an expression of the $Q$ factor in the special case when the ring resonator has negligible loss and weak cross-coupling using Eq. 2.20 and the m-th order resonance:

$$
Q \approx \frac{\pi n_{g} L}{\lambda_{m}} \cdot \frac{t}{1-t^{2}} \approx \frac{n_{g} L}{\lambda_{m}} \cdot \mathscr{F}
$$

Thus a loaded $Q$ factor is proportional to the finesse, and it depends on the structural parameters and dispersion properties of the ring itself. 


\subsection{Time Domain Analysis Method}

Another way to analyze a resonant device, such as the micro-ring resonator, comes from microwave theory [37]. An optical resonant system can be treated like the analysis of a RLC resonant circuit or lumped oscillator [23]. This technique comes from the fact that any resonant system can be described by idealized components that are perturbed or coupled together. The technique is also part of a broader topic known as Coupled Mode Theory, which specifically involves the analysis of energy/power decay rates from resonant systems $[37,38]$. Any decay rate of an oscillator must be understood as power leaving the oscillator.

A resonant system analysis technique also known as Temporal Coupled Mode Theory or CMT consists in analyzing the interaction of a set of elements or blocks that represent localized modes (from a resonant cavity) together with propagating modes (waveguides). In order to obtain a quantitative description of this interaction, the system is parameterized. Two parameters are the resonance frequency and decay rates of the resonant modes, which are assumed to be known a-priori. Usually, those parameters must be calculated previously based on the geometry and material characteristics of the resonant system.

In order to begin with the temporal coupled mode analysis of a ring resonator, we start with an abstract description of a single oscillator. This simple oscillator model is a special case if one considers an add-drop ring resonator as excited with an initial energy with no incident wave at the bus waveguide. In this special case, internal loss is not considered but just the energy transfer from the resonator to the output of the bus waveguide.

An oscillator or resonant cavity has a resonance $\omega_{0}$. Inside the cavity, let's assume the fields are proportional to a complex valued traveling wave $A(t)$. This wave can be obtained by solving the eigen-equation of the optical cavity waveguide. We can also assume

arbitrarily, that this wave has a power $|A(t)|^{2}$, which flows through a cross-section of the 
cavity. For the case of a ring waveguide, the wave $A(t)$ travels around a circumference of radius $R$ and completes one round-trip at a time period $T_{r t}=\frac{2 \pi R}{v_{g}}$; where $v_{g}$ is the group velocity of the modal wave $A(t)$. Finally, based on our previous description, we can obtain the energy stored inside the cavity as $|a|^{2}=|A(t)|^{2} T_{r t}$.

The energy decay rate of the oscillator is related to the power leaving the resonator or coupled into an output waveguide. This decay rate of the mode inside the oscillator can be modeled as a differential equation [37, 38]:

$$
\frac{d a}{d t}=j \omega a-\frac{1}{\tau} a
$$

where $\frac{1}{\tau}$ is the decay rate associated to the power escaping the oscillator and $j \omega a$ is the resonance. The time rate change of the energy can be obtained from the previous equation as:

$$
\frac{d W}{d t}=a^{*} \frac{d a}{d t}+a \frac{d a^{*}}{d t}=-\frac{2}{\tau}|a|^{2}
$$

Therefore the power of the wave is related to energy as:

$$
|A(t)|^{2}=-\frac{2}{\tau}|a|^{2}
$$

Solution of Eq. 2.25 shows the decay of energy of the field inside the oscillator as it transfers its energy to an output waveguide, thus this solution becomes:

$$
|a|^{2}=\left|a_{0}\right|^{2} \exp \left(-\frac{2 t}{\tau}\right)
$$

Instead of energy, we can have a relation in terms of power delivered to the output waveguide $s_{t}$, this is:

$$
\left|s_{t}\right|^{2}=\kappa^{2}|A(t)|^{2}
$$


Where the power delivered to the output is proportional to the power coupled at the interaction region between the oscillator and the output waveguide. The parameter $\kappa^{2}$ is called the power coupling fraction and it is a similar parameter employed in our analysis based on Transfer Matrix Method. As energy and power are related as mentioned in previous paragraphs, then the power at the output waveguide of the oscillator is:

$$
\left|s_{t}\right|^{2}=\frac{\kappa^{2}}{T_{r t}}|a|^{2}
$$

The above expression gives us a relation between energy normalized signals with power normalized signals. The importance of this relation is that it connects the temporal coupled modes method with the commonly known coupling of modes in space, in which the Transfer Matrix method is based upon [23].

The prior analysis until now gave us simple expressions to model the power transfer dynamics of a single oscillator (or resonator). Now, we extend this oscillator model to a ring resonator in add-drop port configuration.

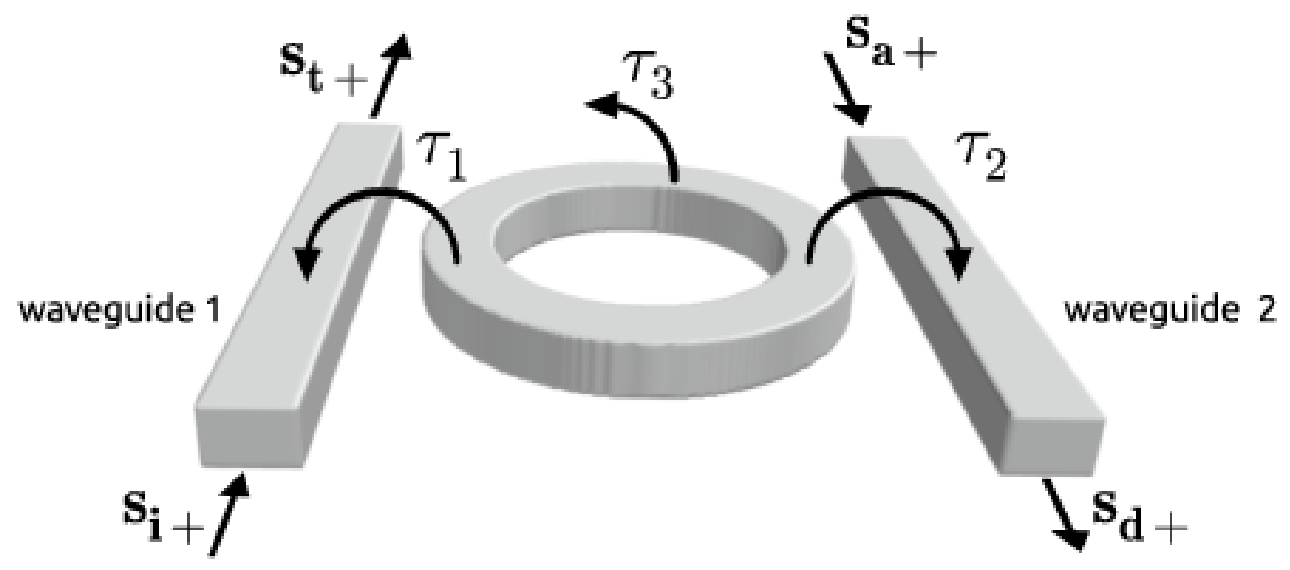

Figure 2.7: Add-drop ring resonator in Temporal Mode Coupling Analysis 
Let's consider a ring resonator device shown in Fig. 2.7. Recalling a ring resonator's analysis using the Scattering matrix approach, the relationship between the incident and transmitted waves is expressed as:

$$
s_{t}=s_{i}-j \mu a
$$

where $\mu$ is a parameter related to the amount of mutual energy coupling between the ring and the mode in a bus waveguide. The mutual energy coupling is defined as $|\mu|^{2}$. As previously mentioned from the single oscillator analysis, the energy coupling is related to the power coupling factor by $|\kappa|^{2}$. Assuming there is no incident wave in the system $s_{i}=0$, then by Eq. 2.31 the power flow to the transmitted output is:

$$
\left|s_{t}\right|^{2}=\mu^{2}|a|^{2}
$$

By inspection of Eq.2.30 and 2.32, the relation between the energy mutual coupling and power coupling factor is:

$$
\mu^{2}=\frac{\kappa^{2}}{T_{r t}}=\kappa^{2} \frac{v_{g}}{2 \pi R}
$$

Finally, we can have a relation between the decay rates and the energy/power coupling factors. Again, looking at Eq. 2.27, we have:

$$
\begin{gathered}
\kappa^{2}=\frac{2}{\tau} T_{r t} \\
\mu^{2}=\frac{2}{\tau}
\end{gathered}
$$

Now, we can start the analysis of the MRR filter employing the oscillator model and decay rates. The time rate change of the energy inside the ring is expressed in a differential equation:

$$
\frac{d a}{d t}=j \omega_{0} a-\frac{1}{\tau_{1}} a-\frac{1}{\tau_{2}} a-\frac{1}{\tau_{3}} a-j \mu_{1} s_{i}-j \mu_{2} s_{a}
$$


The notation used in Eq. 2.35 take into account the field at resonance, the power coupled into the bus waveguide $\left(\frac{1}{\tau_{1}}\right)$, the power coupled into the drop bus waveguide $\left(\frac{1}{\tau_{2}}\right)$, the power lost inside the ring $\left(\frac{1}{\tau_{3}}\right)$ and the energy coupled into the ring from the input ports of the bus and drop waveguides.

We can combine all of the decay rates from the power coupled to waveguide 1, waveguide 2 and intrinsic loss into a single quantity. That is the net lifetime of the oscillator:

$$
\frac{1}{\tau}=\frac{1}{\tau_{1}}+\frac{1}{\tau_{2}}+\frac{1}{\tau_{3}}
$$

Eq. 2.35 can be rewritten using the net lifetime factor and assuming there is no signal entering the ring at the drop port $\left(s_{a}=0\right)$, then:

$$
\frac{d a}{d t}-\left(j \omega_{0}-\frac{1}{\tau}\right)=-j \mu s_{i}
$$

Solution of the above equation for an incident wave $s_{i} \exp (j \omega)$ will give us the field inside the ring $[23,37]$ :

$$
a=\frac{-j \mu}{j\left(\omega-\omega_{0}\right)+\frac{1}{\tau}} \cdot s_{i}
$$

Using Eq. 2.31 we can relate the transmitted wave with the incident wave:

$$
s_{t}=\frac{j\left(\omega-\omega_{0}\right)+\frac{1}{\tau}-\mu^{2}}{j\left(\omega-\omega_{0}\right)+\frac{1}{\tau}} \cdot s_{i}
$$

The energy decay factor is related to decay rates as $\mu^{2}=\frac{2}{\tau_{1}}$ and the previous equation can be expressed as the transmitted wave:

$$
s_{t}=\frac{j\left(\omega-\omega_{0}\right)+\frac{1}{\tau}-\frac{2}{\tau_{1}}}{j\left(\omega-\omega_{0}\right)+\frac{1}{\tau}} \cdot s_{i}
$$


The transmittance of the add-drop micro-ring resonator can be calculated as the power ratio of the transmitted wave with respect to the incoming wave, applying Eq. 2.40 this will yield:

$$
T=\frac{\left|s_{t}\right|^{2}}{\left|s_{i}\right|^{2}}=\frac{\left(\omega-\omega_{0}\right)^{2}+\left(\frac{1}{\tau}-\frac{2}{\tau_{1}}\right)^{2}}{\left(\omega-\omega_{0}\right)^{2}+\left(\frac{1}{\tau}\right)^{2}}
$$

From the last expression, an interesting result is observed if the decay rates are equivalent $\frac{1}{\tau}=\frac{2}{\tau_{1}}$. When the ring is on resonance, the transmittance will go to zero, which is the critical coupling condition. A little more detail on this condition can be extracted if we recall that the net lifetime is $\frac{1}{\tau}=\frac{1}{\tau_{1}}+\frac{1}{\tau_{2}}+\frac{1}{\tau_{3}}$ therefore the critical coupling condition becomes:

$$
\frac{1}{\tau_{2}}+\frac{1}{\tau_{3}}=\frac{1}{\tau_{1}}
$$

The above means that we can extract all power on resonance only if the coupling between the bus waveguide and drop waveguide are not symmetrical even if internal loss (decay rate $\frac{1}{\tau_{3}}$ ) exists in the ring.

Finally, it is preferable to have the transmittance in terms of power coupling coefficients instead of decay rates. Those coefficients can be obtained by mode solving or characterization measurements. From Eq. 2.34, the decay rate of the coupling between the ring and transmission bus waveguide is related to the power coupling factor as:

$$
\kappa_{1}^{2}=\frac{2}{\tau_{1}} T_{r t}
$$

Similarly, by using the symmetry of the add-drop ring resonator structure, the decay rate of the coupling between the modes of the ring and drop bus waveguide is related as:

$$
\kappa_{2}^{2}=\frac{2}{\tau_{2}} T_{r t}
$$


At this point it is important to recall that the optical period is $T_{r t}=\frac{L}{v_{g}}$ where $L$ is the circumference, the group velocity $v_{g}=\frac{c}{n_{g}}$ and $n_{g}$ is the group index in a ring waveguide. Once we put together those factors, we can rewrite Eq. 2.41 to have:

$$
T=\frac{\left(\omega-\omega_{0}\right)^{2}+\left(\frac{v_{g}}{2 L}\right)^{2}\left(\kappa_{2}^{2}+\kappa_{3}^{2}-\kappa_{1}^{2}\right)^{2}}{\left(\omega-\omega_{0}\right)^{2}+\left(\frac{v_{g}}{2 L}\right)^{2}\left(\kappa_{1}^{2}+\kappa_{2}^{2}+\kappa_{3}^{2}\right)^{2}}
$$

It is convenient to express the transmittance equation in terms of optical wavelengths rather than frequencies. The reason is that transmittance is commonly obtained by spectrum measurement equipment, e.g. optical spectrum analyzer or optical power meters with tunable lasers. A detailed explanation of this topic is explored in chapter 5. To do this conversion, first we define $\Delta \lambda=\lambda-\lambda_{0}$ and the frequency:

$$
\left(\omega-\omega_{0}\right)^{2}=\Delta \lambda^{2}\left(\frac{2 \pi c}{\lambda_{0}^{2}}\right)^{2}
$$

Next, the group velocity is related to the free spectral range and the ring circumference as:

$$
\left(\frac{v_{g}}{c}\right)^{2}=\left(F S R \frac{L}{\lambda_{0}^{2}}\right)^{2}
$$

Now, according to the previous relations, the through port transmittance is expressed [32]:

$$
T=\frac{\Delta \lambda^{2}+\left(\frac{F S R}{4 \pi}\right)^{2}\left(\kappa_{2}^{2}+\kappa_{3}^{2}-\kappa_{1}^{2}\right)^{2}}{\Delta \lambda^{2}+\left(\frac{F S R}{4 \pi}\right)^{2}\left(\kappa_{1}^{2}+\kappa_{2}^{2}+\kappa_{3}^{2}\right)^{2}}
$$

The transmittance above now depends on some parameters that can be measured directly like the resonance wavelength and FSR. The power coupling coefficients can also be determined indirectly from measurements by obtaining the insertion loss and through port extinction. 
A similar procedure can be followed in order to get the power detected at the drop port of the micro-ring resonator. Assuming that no signal enters at the add port and taking into account power conservation, the drop port power is:

$$
\left|s_{d}\right|^{2}=\left|s_{i}\right|^{2}-\left|s_{t}\right|^{2}
$$

Using Eqs. 2.41, 2.46 and 2.47, the drop port transmittance is:

$$
D=\frac{4 \kappa_{2}^{2} \kappa_{3}^{2}\left(\frac{F S R}{4 \pi}\right)^{2}}{\Delta \lambda^{2}+\left(\frac{F S R}{4 \pi}\right)^{2}\left(\kappa_{1}^{2}+\kappa_{2}^{2}+\kappa_{3}^{2}\right)^{2}}
$$

The drop port transmittance resembles a Lorentzian shaped distribution, which is like the spectral response of Fabry-Perot optical filters [36]. This spectrum shape will prove its usefulness in this research. In later chapters, simulations of a wavelength sensor interrogation system will assume a filter with Lorentzian shaped spectrum response.

\subsubsection{Ring resonator parameter extraction using CMT model.}

With the Coupling of Modes in Time ring resonator model is possible to extract physical parameters, such as intrinsic loss and power coupling factors, based on direct spectral measurements. This characterization methodology proposed by S. Xiao et al. has the advantage of giving deterministic results, which are independent of the losses present in a typical measurement environment such as the fiber to optical port coupling [32]. In order to extract those parameters, four spectrally measured values must be provided at each resonant wavelength: the minimum power transmission at the through port, the maximum power transmission at the drop port, the average FSR between two resonances, and the $3 \mathrm{~dB}$ bandwidth (FWHM) at each resonant wavelength. 


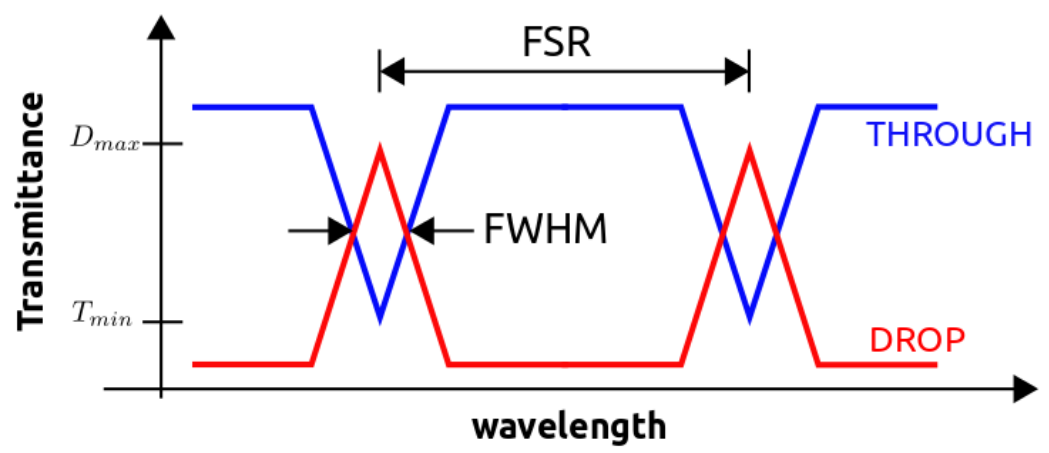

Figure 2.8: Transmittance values for micro-ring parameter extraction based on the Coupling of Modes in Time method.

The basic concept is illustrated in figure 2.8 where the theoretical spectrum response of a ring resonator is sketched and the basic measured values are labeled.

This procedure begins with the through and drop transmittance models from Eq. 2.48 and 2.50. The $3 \mathrm{~dB}$ bandwidth can be extracted using the through port transmittance when its value reaches half its maximum, therefore [32]:

$$
\delta \lambda=\left(\frac{F S R}{2 \pi}\right)\left(\kappa_{1}^{2}+\kappa_{2}^{2}+\kappa_{3}^{2}\right)
$$

An important assumption is that an add-drop ring resonator has symmetrical coupling between the bus to ring and vice versa. This simplifies the modeling if we substitute Eq. 2.51 into the through port transmittance in Eq. 2.48 and assuming that $\kappa_{1}^{2}=\kappa_{2}^{2}$ then we have:

$$
T=\frac{\Delta \lambda^{2}+\left(\frac{F S R}{4 \pi}\right)^{2}\left(\kappa_{3}^{2}\right)^{2}}{\Delta \lambda^{2}+\left(\frac{\delta \lambda}{2}\right)^{2}}
$$

Now, we can obtain the intrinsic loss $\left(\kappa_{3}^{2}\right)$ based on the measurement of the minimum through port power $\left(p_{t}\right)$ at resonance $(\Delta \lambda=0)$, i.e.:

$$
\kappa_{3}^{2}=2 \pi \delta \lambda \frac{\sqrt{p_{t}}}{F S R}
$$


Also, the power coupling coefficients $\left(\kappa_{1}^{2}\right.$ and $\left.\kappa_{2}^{2}\right)$ can be obtained from Eq. 2.51 and 2.53, which give us the following expression:

$$
\kappa_{1}^{2}=\kappa_{2}^{2}=\pi \delta \lambda\left(\frac{1-\sqrt{p_{t}}}{F S R}\right)
$$

With the extracted internal loss, an important observation can also be derived. It is possible to obtain the intrinsic quality factor of the add-drop ring resonator, which gives an upper bound of the amount of energy stored inside the ring.

The $\mathrm{Q}$ factor can be calculated by the definition from Eq. 2.22. The internal energy stored is $|a|^{2}$, and is transferred to the through and drop ports respectively. Therefore, the intrinsic Q can be approximated as [32]:

$$
Q_{i}=\frac{2 \pi \lambda_{0}}{F S R \cdot \kappa_{3}^{2}}
$$

Some comments are worth mentioning. The ratio of the power inside a ring resonator vs. the input power also known as build-up factor is proportional to $\sim 1 / \kappa_{1}^{2}$ [23]. This implies that the light circulating the ring increases its intensity at low input power coupling values (high Q-factor). Silicon presents non negligible third order nonlinear properties, such as two-photon absorption and thermal nonlinear optical effects [7, 39]. Therefore, those nonlinear effects are enhanced inside a high $\mathrm{Q}$ ring resonator filter and both can induce changes in its resonance wavelength because of the high optical confinement and power build-up inside the ring. Those issues are important because they seriously limit the extrinsic Q-factor of the resonator in scenarios when interrogating sensors at short distances or high optical power sources are employed.

In summary, with this method, some interesting physical parameters can be extracted from direct spectral measurements. Coupling losses are expected due to the geometrical differences between a coupling fiber and a silicon optical waveguide and also the index 
difference between silica and silicon. However, the extracted values using the previous method are independent on those light coupling losses in an optical chip. 


\section{CHAPTER 3}

\section{OPTICAL SENSORS: FIBER BRAGG GRATINGS}

\subsection{Introduction}

An optical sensor is a device where a physical/chemical variable produces an alteration of some characteristic of the light propagating through it. Those alterations or modulations modify the amplitude, phase, frequency or polarization of light. Some key attributes of optical sensors are their immunity to electromagnetic interference and their low propagation loss at optical wavelengths. There is a wide array of techniques that use optical sensing, but one type based on measuring wavelength encoded information became very popular in recent years. The fiber Bragg grating sensor is a particular example of an intrinsic optical sensor, which works by measuring its resonance wavelength changes due to any induced temperature or strain perturbations.

Fiber Bragg sensors have been adopted in many applications, such as in civil structures [18], oil well pressure sensing [40], relative humidity measurements [41] and high current/voltage measurements in electrical plants [42] to name a few. It is expected that the optical fiber sensor market could reach $\$ 1.95$ billion in revenues by the end of this decade [13]. Those expectations are based on the fact that fiber Bragg sensors are very versatile devices, which can be adapted to solve control and measurement needs in many industries.

In the following sections, our initial experiment on embedding fiber Bragg sensors in metallic structures through plasma spray is presented, followed by a theoretical analysis of a fiber Bragg sensor and an explanation of its main parameters. A review of common demodulation techniques found in the literature is given, with a special treatment dedicated to a certain demodulation technique based on tunable optical filters. 
Later on, this technique will be useful in explaining our wavelength demodulation system based on a silicon photonic device.

\subsection{Review of Fiber Bragg Grating Sensors}

Fiber Bragg grating (FBG) sensors have a characteristic in which a measurand of interest (strain or temperature) is encoded into an optical wavelength. This feature makes those sensors particularly resilient because measurements do not depend on optical power fluctuations $[43,44]$. Fiber Bragg grating sensors have a comparative advantage over other sensing alternatives, for example, they can be interrogated in a distributed way by employing multiplexing techniques. Furthermore, by embedding FBG sensors on certain materials, one could put "intelligence" into civil structures enabling the concept of "smart structures" [16]. Those types of structures are constructed with the objective to assess the mechanical health, deformation and integrity of a civil engineering infrastructure, with the possibility to integrate actuation devices commanded from feedback control systems.

Fiber Bragg Grating (FBG) sensors are made from a phenomenon called photosensitive effect on doped silica fiber. That effect was discovered by Hill and others in germanosilica optical fibers and it consisted in the change of the refractive index of a fiber core after exposing it to certain amount of UV light [45]. The amount of sensitivity is controlled by higher Ge doping of a standard silica fiber or by hydrogenation and boron doping techniques [45]. There are many fabrication methods of Bragg gratings in germanosilica fibers, such as: interferometric techniques, phase-mask inscribing and point-by-point writing [45]. A main task of those techniques is the control of the spatial modulation of the UV light in order to write or inscribe periodic spatial refractive index variations. In this research, we had the opportunity to work with fiber Bragg grat- 
ings fabricated by our collaborator, Dr. Carmem Barbosa from IEAv, in Brazil using the phase-mask technique [46].

For engineering applications, FBG sensors embedded into new materials structures have received much attention and interest, specially in composite materials based on placing FBG fibers at certain orientations and layering them into carbon/epoxy plies. Our initial experiments with FBG sensors started by embedding those sensors into other materials using the plasma spray technique. The technique consisted in laying fiber sensors along a AISI 1020 steel plate and stuck them into the plate with thermal tape. An aluminum alloy powder feedstock was used with a plasma spray technique as explained in [47]. This allowed a coating deposition of approximately $1 \mathrm{~mm}$ thick on the plate until the fibers were embedded.

Although the process was very interesting to explore, the results were not satisfactory because some fibers broke due to stress and also due to presence of voids between the fiber and steel plates attributed to the motion of the fiber in the high velocity plasma stream during the initial moments of the deposition. Although the process produced a smooth and adherent coating interface between the fiber and the Aluminum alloy in some samples, however, the process was not further investigated because this author had to return to work away from FIU.

Fig.3.1 shows an FBG optical fiber structure with a spatial periodic index of refraction in order to form a grating section along the fiber. The main effect observed after injecting a broadband light source is a narrow-band reflective component at a certain wavelength known as Bragg wavelength. This wavelength is related to spatial index of refraction modulation period $\Lambda$ induced in the effective index at the core $n_{\text {eff }}$ by $[16,15]$ :

$$
\lambda_{B}=2 n_{e f f} \Lambda
$$




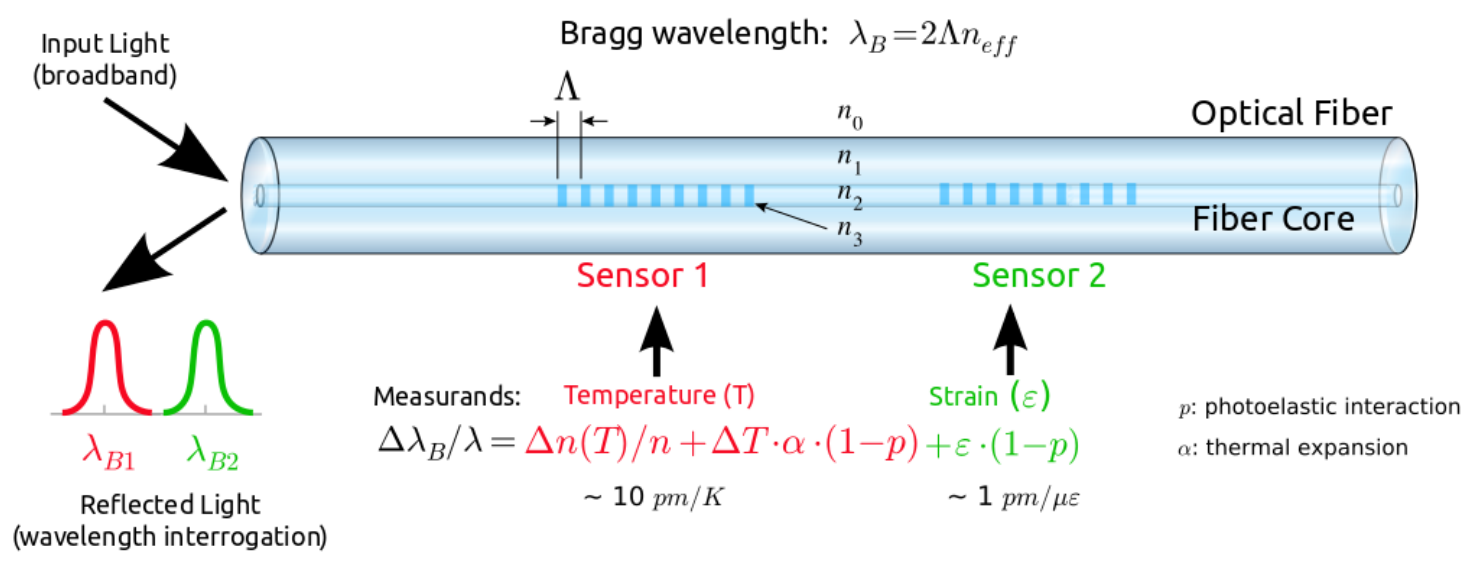

Figure 3.1: Fiber Bragg grating (FBG) sensor main characteristics and its operating principle.

Thus, the Bragg wavelength $\lambda_{B}$ is the center wavelength of the back-reflected light coming from the grating. In next subsections, a discussion of the FBG spectral characteristics and measurand sensitivities are presented, which will give us a view on how an FBG works as a sensor.

\subsubsection{A spectral model of a Fiber Bragg Grating}

One method commonly employed to analyze optical structures, is the Coupled mode theory. This technique is based on a perturbation approach to analyze the interaction of the eigenmodes of uniform dielectric substructures. An uniform periodic change in the refractive index $n_{0}$ of the FBG fiber core can be modeled as:

$$
n(z)=n_{0}+\Delta n \cos \left(\frac{2 \pi z}{\Lambda}\right)
$$

where $\Delta n$ is the modulation amplitude of the UV induced refractive index change and $z$ is the distance along the fiber propagation axis. The evolution of backward and forward propagating modes can be inserted into the coupled mode equations. From the 


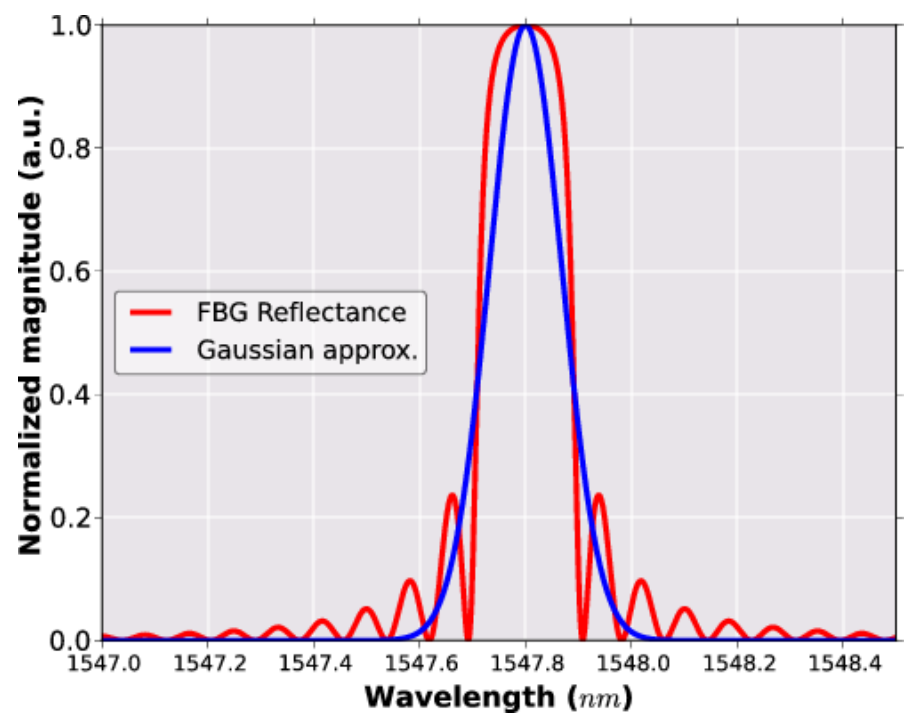

Figure 3.2: FBG Reflectance based on theoretical model from Eq. 3.3 (red color) and approximation using a Gaussian function (blue color). Grating length is $1 \mathrm{~cm}$, grating period is $0.545 \mu \mathrm{m}$, core effective index 1.42 and induced modulation index $\Delta n=1.5 e-$ 4.

coupled mode analysis an expression for the FBG reflectance spectrum is obtained. This reflectivity depends on certain structural parameters $[45,48]$ :

$$
R(L, \lambda)=\frac{\Omega^{2} \sinh ^{2}(s L)}{\Delta \beta^{2} \sinh ^{2}(s L)+s^{2} \cosh ^{2}(s L)}
$$

The reflectivity is a function of wavelength and the "pitch" length of the grating $L$. The parameter $\Delta \beta=\beta-\frac{\pi}{\Lambda}$ called detuning wave vector depends on the effective propagation constant $\beta$ and the modulation period $\Lambda$. The value $s$ corresponds to $\sqrt{\Omega^{2}-\Delta \beta^{2}}$. The parameter $\Omega$ is a transverse coupling coefficient based on the sinusoidal change in refractive index, and can be expressed as:

$$
\Omega=\frac{\pi \Delta n \eta(V)}{\lambda}
$$

where $\eta(V)$ is the fraction of the intensity of the fundamental mode, which depends on the normalized frequency ( $V$-number) of the optical fiber. 
A graph of the reflective response of an FBG is shown in Fig.3.2, the red line corresponds to Eq. 3.3 for a $1 \mathrm{~cm}$ length grating fiber. Side lobes in the spectrum are observed because of the combination of reflections at both ends of the grating. The blue color line represents an approximation of the reflectivity using a Gaussian function model expressed as:

$$
G(\lambda)=R_{0} \exp \left[-4 \ln 2\left(\frac{\lambda-\lambda_{G}}{\Delta \lambda_{G}}\right)^{2}\right]
$$

A Gaussian approximation clearly depends on the height $R_{0}$, the center wavelength $\lambda_{G}$ and the full-width-at-half-maximum (FWHM) $\Delta \lambda_{G}$. Comparing the two curves on Fig.3.2, the Gaussian curve follows the trend of the original FBG but fails to capture its details, such as the side lobes and main lobe width [48]. However, a Gaussian model of the FBG will be important later on when an analysis is required to understand how an interrogation system based on MRR optical devices works.

\subsubsection{Relation between strain and temperature measurands and the spectrum of}

\section{an FBG}

If the mechanical structure of the fiber is modified either by an axial strain or temperature change, those variations induce a perturbation on the periodicity of the index of refraction, which translates into a shift of the Bragg wavelength [16]. When an FBG is interrogated by a light source, the wavelength shift of its reflection spectrum has a linear relationship with temperature and strain [16]:

$$
\Delta \lambda_{B}=2 n \Lambda\left\{\left[1-\left(\frac{n^{2}}{2}\right)\left[p_{12}-v\left(p_{11}+p_{12}\right)\right]\right] \cdot \varepsilon+\left[\alpha+\frac{\frac{d n}{d T}}{n}\right] \cdot \Delta T\right\}
$$


where $n$ is the index of refraction of the fiber core, $p_{12}$ and $p_{11}$ are the Pockel's coefficients, $v$ is the Poisson ratio, $\varepsilon$ is the axial strain, $\alpha$ is the thermal coefficient of expansion of the FBG, $\frac{d n}{d T}$ is the index of refraction change due to temperature and $\lambda_{B}$ is the Bragg wavelength. This relation clearly indicates that information been sensed (strain or temperature) is encoded into the wavelength, which does not depend on light level, losses or source power. That characteristic makes a Fiber Bragg grating fiber a robust and reliable sensor. From Eq. 3.6, the contribution of the thermal expansion of the fiber was not included as the thermo-optic change is a dominant factor [45].

Both measurands in Eq. 3.6 control the amount of wavelength shift of the FBG reflectance or transmittance spectrum. Assuming a constant temperature environment and an isotropic/homogeneous optical fiber, the relative wavelength shift due to strain is [16]:

$$
\frac{\Delta \lambda_{B}}{\lambda_{B}}=\left(1-p_{e}\right) \varepsilon \approx K_{S} \varepsilon
$$

where $p_{e}$ is the photo-elastic constant, and $K_{S}$ is the strain sensitivity factor (strain responsivity) of $0.78 \times 10^{-6} \mu \operatorname{strain}^{-1}$ and $\varepsilon$ is the applied axial strain.

Assuming an arbitrary fixed strain, the wavelength change due to temperature only is described as:

$$
\frac{\triangle \lambda_{B}}{\lambda_{B}}=K_{T} \Delta T
$$

where $K_{T}$ is the temperature sensitivity factor, which for silica fiber have an approximate value of $6.68 \times 10^{-6}{ }^{\circ} \mathrm{C}^{-1}[16][15]$.

On table 3.1, the strain sensitivities values are very similar, but the temperature sensitivities differ among references found in the literature. A possible explanation of this variability could be differences on the chosen FBG germanosilicate fibers. Previously, it was mentioned that the thermo-optic change $\frac{1}{n} \frac{\partial n}{\partial T}$ is a dominant factor compared to the thermal expansion coefficient $\alpha$, this is true for glass standard reference materials (SRM) 


\begin{tabular}{c|c|c|l} 
References & Strain sensitivity & Temperature sensitivity & \multicolumn{1}{|c}{ Wavelength } \\
\hline$[45]$ & $0.7874 \times 10^{-6}$ & $9.15 \times 10^{-6}{ }^{\circ} \mathrm{C}^{-1}$ & $1550 \mathrm{~nm}$ \\
{$[16]$} & $0.78 \times 10^{-6}$ & $6.67 \times 10^{-6}{ }^{\circ} \mathrm{C}^{-1}$ & $1300 \mathrm{~nm}$ \\
{$[49]$} & $0.782 \times 10^{-6}$ & $6.782 \times 10^{-6}{ }^{\circ} \mathrm{C}^{-1}$ & $1535.55 \mathrm{~nm}$ \\
{$[50]$} & $0.769 \times 10^{-6}$ & $8.658 \times 10^{-6}{ }^{\circ} \mathrm{C}^{-1}$ & $1550 \mathrm{~nm}$ \\
{$[15]$} & $0.769 \times 10^{-6}$ & $6.452 \times 10^{-6}{ }^{\circ} \mathrm{C}^{-1}$ & $1300 \mathrm{~nm}, 1550 \mathrm{~nm}$
\end{tabular}

Table 3.1: Comparison of FBG strain and temperature sensitivities found in literature.

as silica, however the concentration of Germanium can modify the overall temperature sensitivity as it was demonstrated experimentally elsewhere [51].

From the above data, an estimation of minimum wavelength resolution required can be obtained. For example, assuming an FBG is operating at $1550 \mathrm{~nm}$ region, a strain sensitivity of $0.78 \times 10^{-6}$, for a $1 \mu$ strain will mean a resolution of $1.2 \mathrm{pm}$ and for temperature sensitivity of $6.7 \times 10^{-6}$ a temperature change of $1^{\circ} \mathrm{C}$ will mean a $10.3 \mathrm{pm}$ resolution. This estimation is important because it will determine the measurement limits of a sensor demodulation system.

FBG sensors have dual sensitivity of strain and temperature. This issue can be detrimental for strain sensing applications specifically in quasi-static measurements, because thermal variations along the fiber introduce strain-like sensor readings. By quasi-static strain measurements we mean a material specimen with quasi-static loading in which the time dependent deformation can be visualized as a sequence of static deformations.

A way to overcome this inherent limitation is to add along an array of sensors a reference FBG that has thermal contact to the array but it is independent to local strain. For example, if two FBG sensors are spliced together, then the overall measurand response can be described in matrix form as [16]:

$$
\left(\begin{array}{c}
\Delta \lambda_{1} \\
\Delta \lambda_{2}
\end{array}\right)=\left(\begin{array}{ll}
K_{S 1} & K_{T 1} \\
K_{S 2} & K_{T 2}
\end{array}\right)\left(\begin{array}{l}
\varepsilon \\
T
\end{array}\right)
$$


From the above system of equation, clearly when one of the sensitivities is zero then the respective FBG sensor can be used as a reference. In case that both sensors have different sensitivities and the determinant of the matrix is non-zero, then inverting their matrix relation yield the strain and temperature of the structure [16].

\subsection{Fiber Bragg Grating sensor demodulation techniques}

The problem of wavelength detection in optical sensors is important especially in Fiber Bragg grating (FBG) sensors where their reflectance spectrum shifts are modulated by a measurand value, such as strain and temperature.

To extract those measurands, a demodulation process is applied, which can be either a quasi-static or dynamic detection process. We define as quasi-static measurement as the one applied to a measurand that changes slowly in time. On the other hand, a dynamic

measurement applies to a measurand that change at a strain rate loading greater $10^{-3} s^{-1}$ [52]. In this section a brief summary of some techniques will be presented.

A common methodology employed in laboratory settings relies on spectral measurements with a monochromator, Optical Spectrum Analyzer (OSA) or grating spectrometer [43] to acquire FBG quasi-static spectral shift measurements.

Another technique known as the ratio-metric approach relies upon the splitting of the light beam into two components, one acting as a reference while the other is filtered with a lossy and wavelength dependent optical component. The measured power ratio of the two components will act as an indicator of the amount of wavelength change $[53,43,16]$. A modification of the ratio-metric approach includes a tunable bandpass filter that converts wavelength shift information into a spatial light distribution characterized by a photodiode array or position sensing device [54]. 
On the dynamic measurement side, one method known as the interferometric approach stands for its high resolution. It is based on an unbalanced Mach-Zehnder interferometer with a mechanically tunable optical filter. This system has a co-sinusoidal transfer function, with its phase dependent on the input beam wavelength. In this system, a shift in wavelength is translated into a phase shift at the output signal [16].

Another dynamic measurement method for FBG sensors incorporates a tunable filter based on either Fabry-Perot (F-P) filter, acousto-optic filters or FBG filters. With a F-P filter, its resonance wavelength is tuned and mechanically scanned via a piezoelectric device. The scanning rate can reach frequencies up to $1 \mathrm{KHz}[16]$. As the filter resonance wavelength is tuned it will eventually match the FBG resonance, therefore whenever there is a match in both wavelengths, the Bragg wavelength can be extracted from the amount of tuning voltage applied to the tunable filter. In a similar fashion it is also possible to employ another FBG fiber but working as a tunable filter. This fiber filter relies on a mechanical stretch using a piezoelectric element to sweep periodically its resonance. With this arrangement, it is possible to read multiple FBG grating sensors colorblue sequentially [55].

Another possible approach is to use a laser to monitor and detect FBG sensor reflectance resonances. That technique applies laser pulses from a fiber laser to interrogate an FBG sensor in which any reflectance wavelength shift is converted into temporal shifts measured by a fast detector and sampling oscilloscope [56].

Recently, another method works with a laser diode and a photo-detector connected to an FBG sensor fiber. The laser acts both as a light source and demodulation system in which a self-mixing effect in the laser can detect wavelength shifts induced by dynamic variations of a strained sensor [57].

However, there are some limitations on the previous methodologies, for example, on the use of optical spectrum analyzers, although they provide high wavelength resolu- 
tions, its measurement response is slow and limited, it has bulk size / weight and requires frequent calibration $[43,57]$. The ratio-metric method in some experiments was implemented using bulk-optic filters and collimation elements, which impacts the system size and requires optical alignment $[43,16]$. Also, its sensitivity is limited due to the optical characteristics of the components [16].

While active scanning methods provide high resolution, however, their implementation also relies on large optical elements. In addition, its measurement speed is slow due to the mechanical movement needed to tune the filters and also they require complicated signal processing for demodulation [53]. While an FBG tuning filter method relies on mechanically strain a fiber Bragg grating using a piezoelectric element, it is limited by the fact that its wavelength scanning range is only a few nanometers [55].

Interferometers based on the Mach-Zehnder structure offer a superior wavelength measurement resolution, but usually they are bulky, expensive and vibration sensitive. Interferometers based on optical fibers are better; however, the main issue is to have clearly defined interference fringes in the output signal. To have that, an interferometer optical path length difference (OPD) must be within the range of the coherence length of the optical signal reflected from the FBG sensor. This issue limits the miniaturization of an FBG interrogation system, because long fibers are needed [16].

\subsubsection{FBG demodulation based on tunable optical filter}

In this subsection, an analysis of the tunable optical filter for optical sensor demodulation will be presented. This will be very useful later on in order to understand the application of a silicon photonic device as a wavelength detection system, and also to develop analytical tools to predict its performance. 
One technique for quasi-static FBG interrogation employs a tunable passband optical filter to scan and track the FBG signal. Types of filters commonly used are Fabry-Perot (F-P) [16], acousto-optic filters [58] and also mechanically actuated FBG fibers [21, 55].

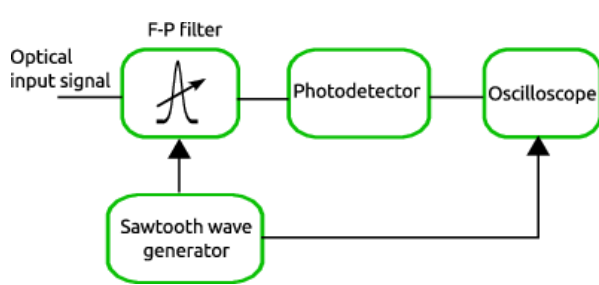

(a)

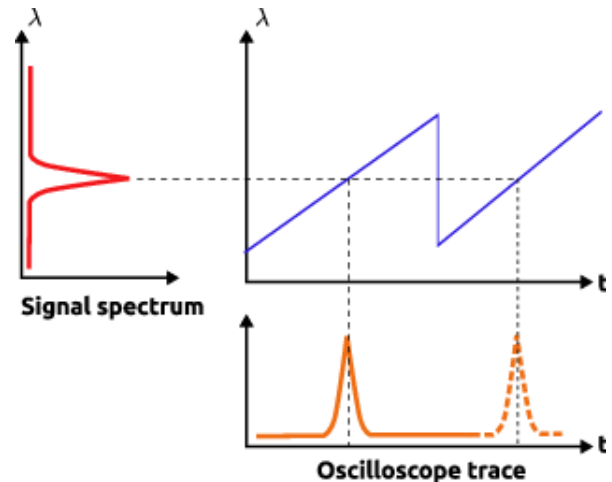

(b)

Figure 3.3: (a) Spectrum measurement using a narrow-band Fabry-Perot tunable filter. (b) Operating principle of the scanning Fabry-Perot spectrum measurement.

Independent of the particular operation of any optical filter, in general, scanning mode interrogation works as shown in Fig. 3.3. Light reflected from an FBG sensor passes through the optical filter. Depending on the construction of the filter, its passband resonance is linearly tuned either electrically or mechanically. The purpose of this passband is to scan over the reflected signal from the FBG. With this method, the FBG spectrum and its center wavelength can be extracted from the voltage or current applied to tune the filter [16]. Usually, the voltage tuning and filter resonance wavelength are linearly related to each other. By linearly tuning an optical filter, reflected light from FBG sensor is scanned and its spectral shape can be obtained, this closely resembles the operation of a monochromator or spectrometer.

To interrogate a group of sensors within a certain wavelength range, a scan filter should have a free spectral range greater than total wavelength covered by the sensors [59], this condition is necessary in order to prevent any spectrum folding or overlap effects that could distort the scanned spectrum [60]. 


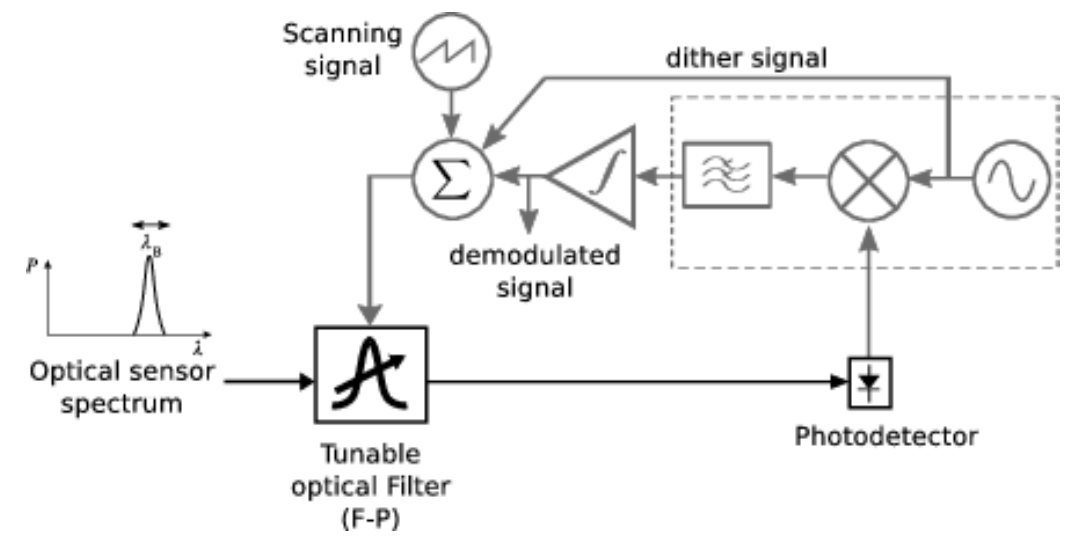

Figure 3.4: A tunable optical filter for FBG sensor demodulation in wavelength tracking operation.

One disadvantage of the scanning filter technique is that only a small portion of the optical spectrum is used. A metric of interest is the detectable energy, which is proportional to the ratio between the filter bandwidth and scanned wavelength range. For example, with a high reflectance FBG, a filter with a bandwidth of $1 \%$ of the scanned spectrum will detect $1 \%$ of the total energy. This result implies that high reflectivity sensors and high optical power sources are needed for good resolution [16].

Scanning or sweep mode detection is a simple and direct way to demodulate any wavelength shift of an FBG sensor however its resolution is limited. A way to detect small shifts in Bragg wavelengths from a single optical sensor is to employ a closed loop tracking system [59]. This tracking system is a type of derivative detection method well known in spectroscopy $[61,62]$.

Tracking mode employs a small dithering signal to modulate an optical tunable filter e.g. a Fabry-Perot or FBG filter. A picture of this type of system is shown in Fig. 3.4. The filter transmittance overlaps with the sensor optical spectrum and resulting light is passed through a photo-detector, which produces an electrical signal. This signal is electrically mixed and filtered together with the same dithering signal. As a result the first harmonic signal (or derivative response) of the detected optical response is obtained [59]. 
The derivative signal is useful because we can track the zero crossings to locate for the peaks or center wavelengths of the FBG sensor. Also, by maximizing the tracking sensitivity, we can determine Bragg wavelength shifts with good resolution.

\subsubsection{Theoretical analysis of scanning and zero-crossing detection methods}

In this subsection, more details on the scanning and zero-crossing detection methods will be explained. The analysis is based on the procedure described in [55, 48] and [63]. The concepts described here are well known in the FBG literature, and the importance for its exposition lies in their use in the simulations performed later on when our ring resonator is used as an FBG interrogator.

We first analyze the scanning operation, which basically employs a ramp signal to linearly tune an optical filter ${ }^{1}$. In this type of operation normally a relation between the optical filter center wavelength and a electrical signal, such as voltage is generally assumed, in other terms: $\lambda_{0}=f(V(t))$, where $V(t)$ is a time domain voltage signal applied to the filter to tune its resonance $\lambda_{0}$. Usually this tuning signal is modeled as a linear equation in time (e.g. a triangular or ramp signal). Therefore, there exists a linear correspondence between the time and the wavelength resonance value of the tunable filter.

In order to make our mathematical analysis tractable, light sources and optical filter transmission spectra are assumed as Gaussian functions. We start by defining the reflectance of an Fiber Bragg Grating sensor (FBG,) which as explained previously in this chapter can be described as:

$$
R(\lambda)=R_{G} \exp \left\{\frac{-4 \ln 2}{B_{G}^{2}}\left(\lambda-\lambda_{G}\right)^{2}\right\}
$$

\footnotetext{
${ }^{1}$ A similar analysis can be done if a multi-mode laser is used.
} 
where $\lambda_{G}$ is the Bragg wavelength of the sensor, $B_{G}$ is the FWHM and $R_{G}$ is the peak reflectance of the sensor.

Transmittance spectrum of some optical filters, such as Fabry-Perot or ring resonators, has a Lorentzian shape at their resonance wavelengths $[53,64]$. However, in this analysis we assume that for a certain wavelength region (less than one FSR), the filter spectrum can be approximated by a Gaussian function. This Gaussian spectrum will be characterized by its height $\left(T_{R}\right)$, spectral width or FWHM $\left(B_{R}\right)$ and center wavelength $\left(\lambda_{0}\right)$. Thus an expression can be defined as:

$$
T(\lambda)=T_{R} \exp \left\{\frac{-4 \ln 2}{B_{R}^{2}}\left(\lambda-\lambda_{0}\right)^{2}\right\}
$$

Notice that a Gaussian spectrum was also an approximation of the reflectance of an FBG, which can act as a matched filter. The use of an FBG filter technique is well known and documented in the literature.

Let's assume to simplify calculations that the FBG sensor is illuminated by a spectrally constant or flat laser source $S_{\lambda}$. As the optical filter is tuned, its resonance changes and its spectrum overlaps with a light source or optical sensor. An integral between both spectra give us a measure of the total light intensity that can be collected by a photodetector [63]. The total light intensity can be expressed in close form equation by an integral. An expression for the spectrum overlap is:

$$
I=\alpha S_{\lambda} \int_{-\infty}^{\infty} R(\lambda) T(\lambda) d \lambda
$$

where $\alpha$ is a parameter that accounts for any coupling loss along the optical system. Inserting equations 3.10 and 3.11 into 3.12 will yield:

$$
I=\alpha S_{\lambda} \int_{-\infty}^{\infty} R_{G} T_{R} \exp \left\{\frac{-4 \ln 2}{B_{G}^{2}}\left(\lambda-\lambda_{G}\right)^{2}-\frac{4 \ln 2}{B_{R}^{2}}\left(\lambda-\lambda_{0}\right)^{2}\right\} d \lambda
$$


To solve analytically this integral and after some algebra we obtain a form like this:

$$
I=\alpha S_{\lambda} R_{G} T_{R} \int_{-\infty}^{\infty} \exp \left\{-\left(\frac{4 \ln 2}{B_{G}^{2}}+\frac{4 \ln 2}{B_{R}^{2}}\right) u^{2}+2\left(\frac{4 \ln 2}{B_{R}^{2}} \Delta \lambda\right) u-\left(\frac{4 \ln 2}{B_{R}^{2}} \Delta \lambda^{2}\right)\right\} d u
$$

where we introduced a new integration variable $u=\lambda-\lambda_{G}$ and the wavelength difference $\Delta \lambda=\lambda_{0}-\lambda_{G}$. The solution from the previous integral have a closed form found in some Calculus books [65]. Thus, the intensity can be compactly written as [55]:

$$
I(\Delta \lambda)=I_{0} \exp \left\{\frac{\Delta \lambda^{2}}{A}\right\}
$$

In that formula, to simplify any further mathematical analysis, we have encapsulated some of the constants into new parameters, such as: $I_{0}=\frac{\alpha \sqrt{\pi} S_{\lambda} R_{G} T_{R} B_{G} B_{R}}{\sqrt{4 \ln 2\left(B_{G}^{2}+B_{R}^{2}\right)}}$, and $A=-\frac{B_{G}^{2}+B_{R}^{2}}{4 \ln 2}$.

We can notice from equation 3.15, that a detected intensity has its maximum value of $I_{0}$ when $\Delta \lambda=0$ or the central wavelength of the filter match the one at the FBG sensor. Additionally $I_{0}$ depends on some of the key parameters of the FBG and tunable filter spectrum, such as, their respective FWHM, peak reflectivity $R_{G}$ and peak transmission of the filter $T_{R}$ and the wavelength difference. As expected, the resulting shape of this expression is also a Gaussian function. It is worth mentioning that if we have chosen a different spectrum definition for the tunable filter e.g. a Lorentzian function; the resulting shape will be a convolution between a Lorentzian and Gaussian shapes. This type of shape was well studied in spectroscopy and it is known as a Voigt profile [62], and it only can be expressed in numerical terms.

In order to obtain the wavelength information from the scanning filter method, it is necessary to have a calibrated relationship between the applied tuning voltage vs. wavelength [55]. Usually, a ramp signal is employed to tune a F-P or FBG filter resonance, this 
will make the tuning resonance linearly dependent on time. Thus, it is possible to correlate time instants with a wavelength value. In simple terms, this means that the scanned signal measured by this method and described by Eq. 3.15 will be an approximation of the wavelength domain spectrum. From here, there are several signal processing techniques to post-process the scanned spectrum in order to detect the center wavelength of optical sensors, such as: detecting the peak or maximum, the centroid approach, applying the cross-correlation method and applying polynomial or Gaussian curve fitting algorithms $[66,67]$.

The scanning method can also be applied using a laser source. In [63] a process to measure the wavelength change in an optical sensor was demonstrated by monitoring a tunable laser bias current. The approach is similar as monitoring the tuning voltage applied to an optical filter.

As a final remark, a wavelength scanning or monitoring process is limited in terms of a minimum resolvable wavelength shift [16], because it depends on the resolution of the scanning process, noise, bandwidth of the detector as well as the spectral characteristics of the tunable filter and the sensor signal [68]. From Eq. 3.15, the resolution of the scanning operation depends on the resolution of the filter $\left(B_{R}\right)$, which usually is smaller than the spectral width of the sensors signal $\left(B_{G}\right)$.

Until this part, the wavelength scanning approach allowed us to obtain the spectrum of a sensor signal like an spectrometer. That technique is suitable to measure spectral shifts of FBG sensors in the quasi-static regime. In order to measure fast $\mathrm{AC}$ spectral shifts in a sensor, a tracking mechanism is employed $[59,58,63,55]$. This is based on taking the derivative of the intensity signal and detecting any zero-crossings from that signal. The key advantage here is that noise outside the bandwidth the tracking system is filtered out.

In the wavelength tracking detection approach, a dithering signal is added to the tuning signal used for spectrum scanning. This enables us to obtain a first harmonic approxima- 
tion of the total light intensity. A way to implement the technique is using an amplifier locked to the dithering signal. The first harmonic approximation is also equivalent to a first derivative of the total light intensity being detected. The importance of having a first derivative signal is that the intensity signal maximum (or minimum) can be easily detected by looking at zero-crossings signal events.

Recalling Eq. 3.15, the measured intensity was a function of the wavelength difference $\Delta \lambda$, we continue the analysis by introducing a definition for the dithering signal modeled as a sinusoid [55]:

$$
\Delta \lambda(t)=\lambda_{0}-\lambda_{G}=\bar{\lambda}+\Delta \lambda_{m} \sin \omega t
$$

where $\bar{\lambda}$ is the average difference between the FBG center wavelength and the tunable filter resonance, and $\Delta \lambda_{m}$ is the dithering signal amplitude.

Now, once those new definitions were introduced, we can insert them into the intensity formula from Eq. 3.15, and obtain a periodic function, denoted as:

$$
I(\Delta \lambda(t))=I_{0} \exp \left\{\frac{\left(\bar{\lambda}+\Delta \lambda_{m} \sin \omega t\right)^{2}}{A}\right\}
$$

Again, it is interesting to note that any detected intensity processed by a lock-in amplifier locked to a dither signal, has a similar operation as taking the first derivative of the detected intensity. One way to express a derivative operation is by analyzing the intensity signal in Equation 3.17 using a first harmonic approximation. There are two mathematical approaches to obtain this approximation. One employs Taylor series expansion around the wavelength difference between the optical sensor and the tunable filter center wavelengths respectively, in other words, as a function of $\bar{\lambda}$. The second approach (which is closer to the lock-in amplifier operation) employs a Fourier analysis technique [55, 63]. It is important to know that the amplitude of the first harmonic corresponds to the lock-in amplifier output. 


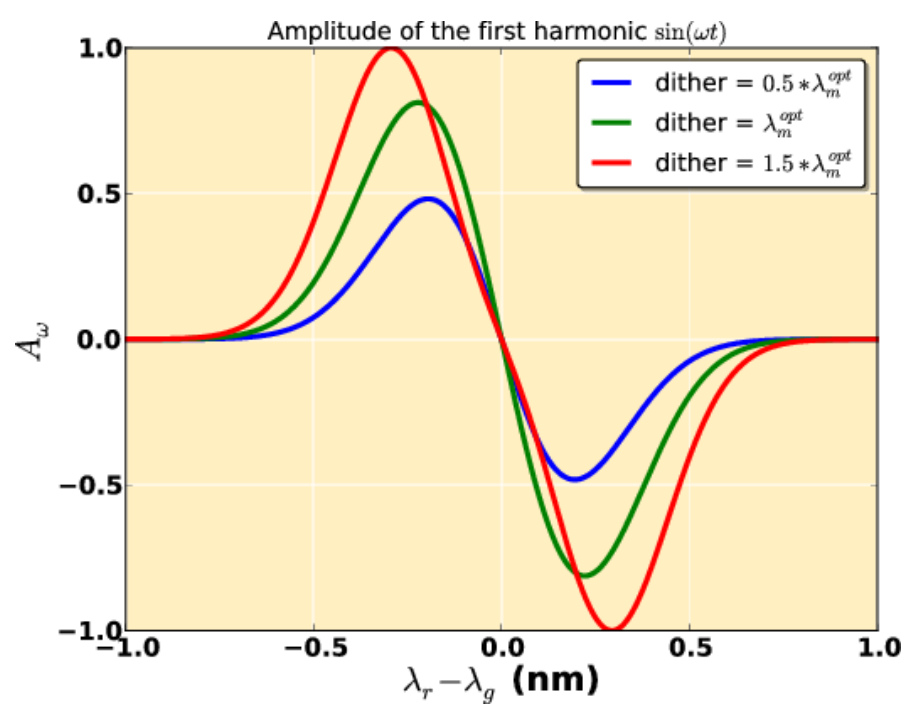

Figure 3.5: First derivative of the Optical Intensity signal obtained at the output of lock-in amplifier.

The following expression is the AC component of the first harmonic using the Taylor series expansion of the periodic intensity. This expression only considered elements up to $\Delta \lambda_{m}^{3}[55]:$

$$
I_{a c}(\Delta \lambda)=I_{0} \exp \left\{\frac{\bar{\lambda}^{2}}{A}\right\}\left[2 \frac{\bar{\lambda}}{A} \Delta \lambda_{m}+\frac{1}{2}\left(\frac{3 \bar{\lambda}}{A^{2}}+\frac{2 \bar{\lambda}^{3}}{A^{3}}\right) \Delta \lambda_{m}^{3}\right] \sin \omega t
$$

The importance of the previous equation can be visualized if we consider a graph of the amplitude of the $\mathrm{AC}$ component $A_{\omega}(\bar{\lambda})$ with respect to the average wavelength difference. Figure 3.5, shows the shape of the amplitude. Whenever the difference between the tunable filter and FBG center wavelengths is zero (both wavelengths match) then there is a zero-crossing event. This event denotes that a peak was detected (zero slope), and as described in $[22,69,55]$, it can be used to measure the strain experienced by an FBG.

From the figure above, the amplitude of the first harmonic (first derivative of Intensity signal) has different slopes around the zero difference region. There is an optimal value that maximizes the slope, which can be calculated by taking derivatives of Eq. 3.17. 
Thus, the optimal dither signal amplitude is:

$$
\Delta \lambda_{m}^{o p t}=\sqrt{\frac{B_{R}^{2}+B_{G}^{2}}{9 \ln 2}}
$$

By maximizing the slope, it is possible to detect the center wavelength of a optical sensor by tracking the zero crossing and it will be less sensitive to errors. In the next section, a tracking system model will be presented and an implementation of this method using a micro-ring resonator device will be described in next chapters.

\subsubsection{Towards a Wavelength Tracking Model}

Tracking or close loop operating method is a process that locks an interrogating laser source (or an optical filter) to an optical sensor center wavelength [63]. To obtain a linear model of the tracking system, we follow a similar analysis done by M.G. Xu et al. [58], which can be applied to any optical tunable filter structure.

In previous paragraphs, a mathematical analysis was presented, which allowed us to have an expression for the first harmonic or derivative of the detected intensity signal. This derivative signal reaches a zero value whenever the optical sensor or laser center wavelength matches the tuning filter resonance. If we bias or tune an optical filter in such a way that we reach the lock region of the tracking system and assume that the wavelength difference between the measured spectrum and tunable filter resonance is small, then our tracking system (lock-in amplifier) will become a wavelength discriminator.

This discriminator is directly proportional to the difference in center wavelengths between the target spectrum and the optical filter. As it was shown in Fig. 3.5, there is a

small region near $\bar{\lambda}=0$ where the derivative signal have a linear relation. This defines the tracking region of a wavelength discriminator. 


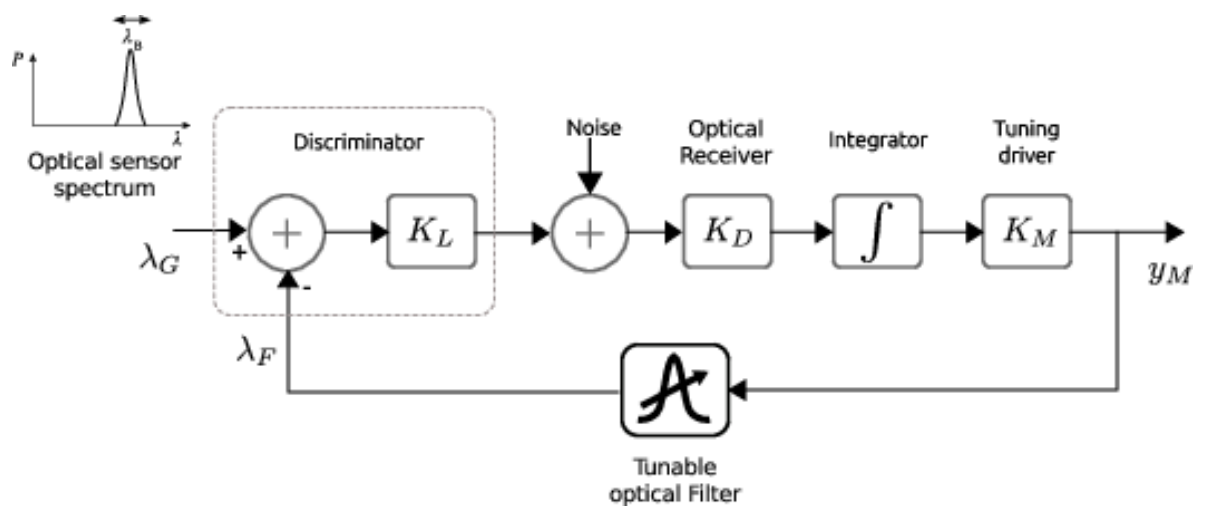

Figure 3.6: Linear system model of a wavelength tracking system employing a tunable optical filter.

A cautionary note must be given for this linear tracking region assumption. We must remember that we assumed a linear relation between the tuning signal and the wavelength center of the filter, it was reported that this type of relation usually is not quite linear but polynomial [70][71] for Fabry-Perot optical filters. Therefore, it is assumed that for other type of optical filters non-linearities may arise and could cause erroneous wavelength readings in the discriminator.

Fig. 3.6 shows a block diagram of a linearized wavelength tracking system based on [58] but applicable to any tunable optical filter independent on its mechanism. The system input is the target spectrum center wavelength (e.g. FBG Bragg wavelength $\lambda_{G}$ ) and the output is the tuning signal, which depending on a filter tuning mechanism it could be a voltage, current or frequency signal.

The optical receiver, integrator and a linearized tuning driver system are modeled as gain blocks with system specific scale factors. For example, the receiver has a photodetector and its light to current conversion is dependent on the responsivity $\left(K_{D}\right)$ of the device. The optical filter is also modeled as a linear system, which converts a certain tuning signal into a filter resonance shift $\left(\lambda_{F}\right)$.

Noise in the system is additive to the detection signal and it can be modeled as a thermal and/or shot noise process in order to mimic a typical photo-detection circuit. 


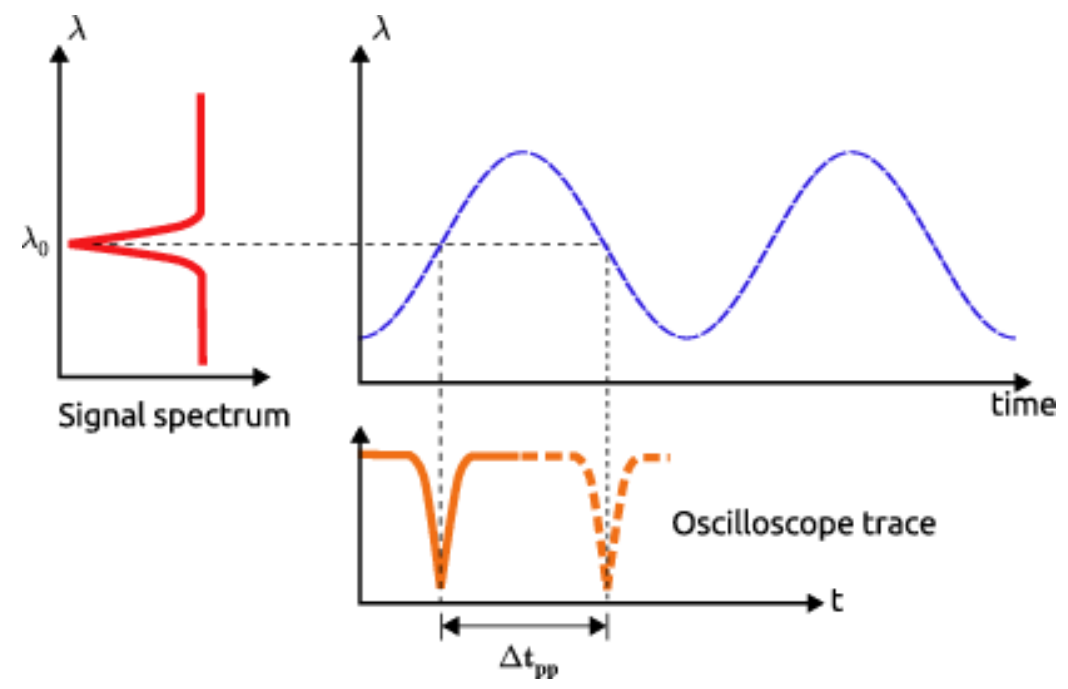

Figure 3.7: Principle of operation of the time-interval between minima (or maxima) measurement method.

The overall response is like a first order loop system, the $3 \mathrm{~dB}$ bandwidth depends on the scale factors of each subsystem block (as well as the lock-in time constant). With this transfer function, the step and sinusoidal response can be easily extracted. Sinusoidal response is limited by the total loop bandwidth, thus in order to track wavelength changes in a target spectrum, the rate of those changes must be less than the loop bandwidth.

\subsubsection{Wavelength center demodulation by time interval measurement method}

With a tunable optical filter in scanning mode, it is also possible to apply a signal processing technique that allows correlating the spectral position of an optical sensor with a time interval measurement. Original research from Mora et al. [21] employed an FBG filter bonded in a piezoelectric element that mechanically tune the filter in time allowing to scan a spectrum region where a desired FBG sensor or light source beam is located.

The basic operation of this demodulator is fairly simple, as it is shown in Fig.3.7. For simplicity, the spectrum of the sensor and FBG filter were modeled as Gaussian shaped functions. In this method, an FBG filter in transmission mode worked as a tunable filter 
by mechanically stretching a piezoelectric (PZT) mechanism. As the FBG Bragg resonance change is proportional to the amount of the PZT mechanical strain, then the FBG resonance wavelength will follow the driving signal of the PZT.

A sinusoidal voltage is employed to modulate a piezoelectric(PZT) fiber stretcher, this type of modulation is chosen because a PZT device has a limited frequency response due to its mechanical resonances. Another reason is that usually a PZT mechanism present intrinsic material hysteresis, therefore by using sinusoidal signals the overall system response will not be affected.

The optical sensor and FBG filter spectra overlaps as the filter is tuned obtaining an intensity, which can be converted to an electrical signal by a photo-diode. This detected signal will show "valleys" or minima on an oscilloscope. The time difference between those minima is a function of the wavelength difference between the optical sensor and the FBG filter.

Advantages of this method were its very high sensitivity $(32.5 \mu \mathrm{s} / \mathrm{pm})$ at the linear region, which is translated into picometer resolutions if the system is calibrated with a narrow-band laser source [21]. Another outcome was that resolution was independent of the intensity of the light source. Some limitations of this method were that a time interval and wavelength response have a non-linear relation at large wavelength changes due to use of sinusoidal PZT tuning. Wavelength scanning range was limited up to 1 $\mathrm{nm}$ because of it was very difficult to increase the maximum voltage excursion to drive the PZT. In addition, long term stability can be compromised due to small deformations of the bonded fiber to a piezoelectric element. Nevertheless, this method stands for its simplicity in implementation and because it measures the time interval between minima or peaks, it is a form of relative measurement suitable for tracking wavelength shifts. 


\section{CHAPTER 4}

\section{DESIGN OF A SILICON PHOTONIC FILTER DEVICE}

With the recent availability of new and advanced lithographic fabrication technologies, it became possible to design and fabricate high index contrast silicon photonic devices, such as a micro-ring resonator. In this chapter, we present this concept for the first time in the design of a silicon photonic sensor interrogation device. First a system overview will be described in order to start with a feasible operating scenario for a photonic optical filter. Then, details are explained of the optical design of a silicon micro-ring resonator. Some design assumptions were made in terms of Q factors, finesse, and waveguide aspect ratio, which resulted in finding the appropriate geometric parameters of the device.

\subsection{Demodulation system characteristics / constraints / parameters}

As mentioned earlier in the previous chapter, the aim of this study was to have a system that interrogates a collection of fiber Bragg grating (FBG) sensors by extracting measurand values, such as strain or temperature. To accomplish that we need a way of detecting any shift in the resonance wavelength of those sensors. This system employs a silicon photonic device known as the micro-ring resonator to do this operation. On this section and the following, a design strategy is described in order to obtain system and device parameters suitable for sensor interrogation.

One important thing to consider is the operating wavelength range of the overall system. FBG sensors operate in different optical bands ranging from $850 \mathrm{~nm}$ to $1675 \mathrm{~nm}$. However, most common FBG optical devices are found in the optical band known as C-band (1530 to $1565 \mathrm{~nm}$ ). One reason why FBGs are on that band is because major demand of FBGs is still found in telecommunication industry, which is a major economic sector in the world. This situation does not only apply to FBG fibers but also to laser 


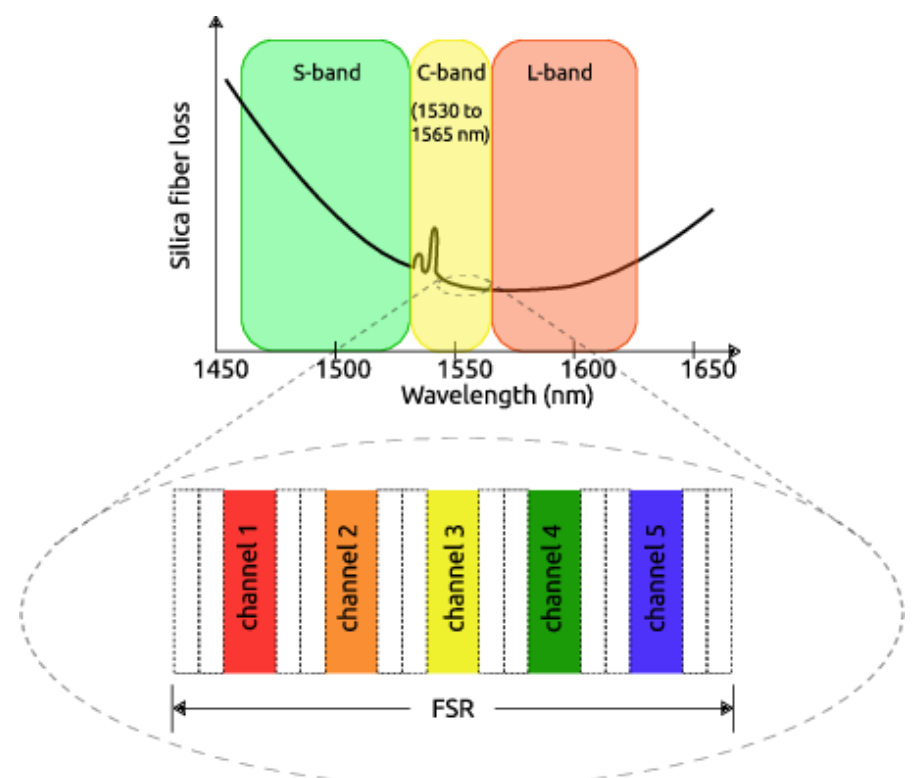

Figure 4.1: Wavelength Division Multiplexed sensor band allocation. The zoomed region corresponds to sensor channels covering the ITU-T C-band wavelength region. Adapted from [10].

sources, photo-detectors, optical amplifiers and optical passive devices. In addition, our previous research $[3,5,20]$ were focused on the C-band for telecommunication and sensor applications. A second argument in favor of using the C-band is that as one of many optical bands, light propagation through optical fibers experience low attenuation at large distances. Then, FBG's operating at that band are suitable for sensing at remote locations. Therefore, this design uses a wavelength range of $35 \mathrm{~nm}$ in the C-band.

A micro-ring resonator (MRR) device works as an optical multiplexer/demultiplexer in a wavelength division multiplexing (WDM) system. This feature is useful in our interrogation system in a way that a number of sensors are individually interrogated and their information can be extracted independently from each other [72].

One issue to mention is how many sensors can be interrogated in a WDM scheme. The answer depends on some factors: 1) the operating wavelength range (35 $\mathrm{nm}$ for this design), 2) the channel spacing, which depends on how much each FBG center wavelength will shift. A simple assumption is that each FBG sensor reflectance do not overlap 
spectrally and each sensor channel will have a certain amount of guard bands. A picture of this allocation scheme is shown in Fig. 4.1. That scheme reserved $3 \mathrm{~nm}$ of FBG wavelength shift range, which in theory means approximately a measurement range of 2400 micro-strain or $280{ }^{\circ} \mathrm{C}$ of temperature per channel. A guard band of $2 \mathrm{~nm}$ per channel was arbitrarily selected. In total, five channels can be allocated in a C-band. However, this simple scheme can be modified accordingly depending on any specific sensing needs or the tolerances of the amount of spectral overlap among sensors.

In terms of the sensor characteristics, two main FBG parameters for system design are the bandwidth (line-shape) and the reflectivity. A study involving tunable optical interrogators (discriminators) showed that when a FBG sensor and tunable filter have the same optical bandwidth, system performance is maximized [55]. Typical values of FBG sensor bandwidth $(F W H M)$ are between 0.1 to $0.3 \mathrm{~nm}$. For our design purposes, the MRR device spectral symmetry and bandwidth are two important attributes to avoid signal distortion and improve demodulation measurement accuracy. In addition, the reflectivity of a FBG sensor is another important factor because higher values (greater than $80 \%$ ) will ease signal detection due to greater spectral selectivity when a tunable filter interrogates an optical sensor.

Continuing with this system design, two additional system components are a light source and photo-detector. An adequate light source is important because it controls the spectrum characteristics of both a FBG and optical device. Some light source attributes are its power level, spectrum bandwidth, polarization and coherency. Specifically, optical sources can be tunable or fixed at certain wavelength or frequency. Tunable sources though, are quite expensive and require complex control to minimize wavelength and power fluctuations. On the other hand, broadband light sources, such as superluminiscense LEDs (SLED), are becoming popular in optical sensor interrogation systems $[73,74]$. SLEDs are available in the C-band and have spectral bandwidths ranging from 
30 to $40 \mathrm{~nm}$ with small form factors and packaged with fiber optic cables that can be easily connected to other optical devices. Commercially available SLEDs have optical powers from $10-12 \mathrm{~mW}$. An important thing to notice on broadband sources is their power density in $\mathrm{mW} / \mathrm{nm}$. A higher value is desirable in order to increase the signal to noise ratio $(\mathrm{SNR})$ at the receiver.

Choosing a photo-detector for the interrogation system will depend on certain parameters like its spectral responsivity, which characterizes how much electrical photo-current is generated per incident optical power at certain wavelength [75]. Another parameter is the Noise Equivalent Power (NEP), which accounts for the lowest power detected under optimum conditions at $1 \mathrm{~Hz}$ bandwidth. This variable is important in the design of any electronic detection circuit. As an example, for a commercial photo-detector sensor (Newport 818-IR), its NEP is approximately $0.7 \mathrm{pW} / \sqrt{\mathrm{Hz}}$. Finally, the Detector bandwidth is a parameter, which depends not only on the photo-detector device but the electronic detection circuit employed.

There is the possibility to choose a customized design that incorporates an embedded photo-detector in the optical chip, using Ge over Silicon technology as the one provided by LETI semiconductor facilities [76]. The advantage of that approach consists in improved packaging, reduced cost and compactness in a way that any optical to electrical conversion can be done in a single chip without external opto-electronic components. Although, Ge over Silicon is attractive, for this design specifically, we opted on the use of external components to avoid further complications and issues from device fabrication.

For this system analysis, it is important to obtain an estimation of the available optical power budget. As mentioned before, a SLED broadband source has the advantage in terms of adequate cost and packaging. Assuming a commercial SLED of $15 \mathrm{~mW}$ with 30 $\mathrm{nm}$ bandwidth, then a spectral power density of the light source is $0.5 \mathrm{~mW} / \mathrm{nm}$. With a single FBG sensor of $80 \%$ reflectivity and 0.1 to $0.2 \mathrm{~nm}$ bandwidth the input power at our 
demodulation system can have a value from -14 to - $11 \mathrm{dBm}$. That range does not consider propagation, connector and splicing losses, which affect system performance. Also, in this system analysis, the use of commercial photo-detector and power meter was preferred for the sake of simplicity. The reason for the use of commercial equipment is to determine a baseline metric of the minimum resolvable power of a photo-detector and the detection meter sensitivity. Using a Ge photo-detector with NEP of $0.7 \mathrm{pW} / \sqrt{\mathrm{Hz}}$ at a maximum detection bandwidth of $10 \mathrm{KHz}$ gives us a estimated value of $-61 \mathrm{dBm}$ [77]. With that data our estimated power budget margin is around $50 \mathrm{~dB}$. Indeed, this estimation does not consider losses in the system and most importantly a micro-ring resonator insertion loss and optical connection losses to the device, but it will give us an idea of how much operating margin we have for our design. If necessary, optical amplification may be needed to overcome any loss in the system.

\begin{tabular}{|c|c|}
\hline Component / Parameter & Value \\
\hline \hline Optical Source (SLED) & \\
\hline Max. output power & $11.7 \mathrm{dBm}$ \\
\hline Spectral width (FWHM) & $30 \mathrm{~nm}$ \\
\hline Optical band & C-band $(1530-1565 \mathrm{~nm})$ \\
\hline Sensor (FBG) & $80 \%$ \\
\hline Reflectivity & $0.1-0.2 \mathrm{~nm}$ \\
\hline Spectral width (FWHM) & $-10.9 \mathrm{dBm}$ \\
\hline Max. reflected power (1) & \\
\hline Photo-detector & $0.7 \frac{\mathrm{pW}}{\sqrt{\mathrm{Hz}}}$ \\
\hline NEP & $10 \mathrm{KHz}$ \\
\hline Bandwidth & $0.8-0.9 \mathrm{~A} / \mathrm{W}$ \\
\hline Responsivity & $-61 \mathrm{dBm}$ \\
\hline Sensitivity (2) & $50 \mathrm{~dB}$ \\
\hline \hline Power budget (1) - (2) & \\
\hline
\end{tabular}

Table 4.1: System components and power budget. 
To summarize our system components design, table 4.1 shows some of the basic parameters with their respective values. Those requirements will help on establishing the starting point on the design of the optical micro-ring resonator device.

\subsection{Optical device design}

In the Introduction chapter of this work, it was mentioned that a key element for a sensor interrogation system is a Silicon micro-ring resonator. Silicon photonics is a proven technology used in the fabrication of optical devices working in the optical telecommunications C-band [2, 7, 78]. Many high technology companies, such as Intel [79], Luxtera [80], Kotura [81] and Caliopa [82] to name a few are currently applying this technology and offering products for data and computer communications.

This section deals with the optical design of a micro-ring resonator that will work as a tunable filter. The ring resonator is going to operate with resonances wavelengths selected around a band of interest of 1530 to $1565 \mathrm{~nm}$. In the research literature, that region is a typical operating band for Silicon photonics devices.

A device parameter of importance is the free spectral range (FSR), which determines the wavelength separation between resonances. Specifically for this interrogation system, the MRR device will act as a filter with its transfer function overlapping the spectrum where the FBG sensor reflectance is located. In order to process each sensor by their respective MRR filter, each MRR resonance must be within each sensor allocated channel and should not overlap with other adjacent sensors. This will impose an FSR that should reach $35 \mathrm{~nm}$ to avoid other MRR resonances from each ring resonator to overlap and interact with other FBG sensors. This requirement is met by either reduce the radius of a ring resonator or employing the Vernier effect by serially coupling multiple rings [83]. In our research done in collaboration with W. Fegadolli et al., we were able to 
demonstrate Vernier effect by adjusting the phase matching between two serially coupled ring resonators using micro-heaters on top of them [84].

Single mode operation is a requirement in order to avoid modal losses, and for a high index contrast waveguide made of silicon over silicon dioxide, light is well confined in the silicon core. This optical confinement enables high optical device integration. To get single mode operation at C-band wavelengths, dimensions of the optical waveguide must be engineered. The ratio of a waveguide width with its height $(w / h)$ known as aspect ratio determines the polarization dependence of the silicon micro-ring device. However, in MRR devices single polarization operation is very challenging due to stringent fabrication tolerances [85].

The MRR tuning range is a device parameter of interest, directly related to what was discussed about how much a FBG sensor center wavelength changes due to strain or temperature. A $3 \mathrm{~nm}$ FBG shift was mentioned as allocated for each sensor channel. For tuning we employ the thermo-optic effect using a micro-heater on top of the ring waveguide device in order to control the temperature and change the MRR resonance. A complete development will be presented in next sub-sections.

\subsubsection{Optical waveguide characteristics}

The design of a micro-ring resonator begins with the selection of a waveguide dimensions and materials. A silicon core surrounded by silicon dioxide ( $\mathrm{SiO} 2)$ cladding will confine light within the core with low optical propagation losses.

Electromagnetic wave components, such as the Electric field (E-field), propagate through a waveguide at certain polarizations. The Transverse Electric (TE) polarization corresponds to an E-field with a major vector parallel to the plane of the optical chip device. The Transverse Magnetic (TM) polarization corresponds to the E-field with a ma- 
jor vector perpendicular to the plane of the chip. Usually, high index contrast dielectric waveguides have hybrid polarizations, meaning that they usually have small E-field vector component either perpendicular or parallel to the plane of the chip. Therefore, those types of waveguides normally have quasi-TE and quasi-TM polarizations.

Our silicon micro-ring design will start by fixing some waveguide parameters that are closely related to the technology applied by silicon photonics fabrication facilities. One of those parameters refers to the waveguide aspect ratio $(w / h)$. In our research group at FIU, a cross-section of $450 \mathrm{~nm} \times 250 \mathrm{~nm}$ was chosen, which assures single mode operation at $1550 \mathrm{~nm}$ wavelength [3]. Waveguide cross-section as the previous mentioned corresponds to an aspect ratio of 1.8 . This aspect ratio is commonly used in research $[9,10]$ and has the advantage of potentially reaching a high FSR of $44 \mathrm{~nm}$ with high quality factors and maximum confined single mode TE excitation [10]. The lower cladding of the waveguide is assumed to be a Buried oxide layer (BOX) with a thickness of $3 \mu \mathrm{m}$, the top cladding normally is silicon dioxide deposited by plasma enhanced vapor deposition (PECVD) with a selected thickness of $1.5 \mu m[3,86]$.

To calculate the waveguide dispersion or the effective mode index at the C-band wavelength range, we employed material data models published in literature. Those models were based on experimental results fitted with a Sellmeier's equation and allow us to determine the material dispersion of silicon and silicon oxide and its dependence on temperature [86].

The picture above shows the refractive index for Silicon (Fig. 4.2(a)) and silicon dioxide (Fig. 4.2(b)) covering S, C and L-band and ranging from room temperature up to 100 ${ }^{\circ} \mathrm{C}$. The maximum temperature was chosen for thermo-optical operation of a waveguide to account for a maximum of $8 \mathrm{~nm}$ tuning range [10]. We notice that silicon refractive index is highly sensitive to temperature variations compared to silica. As mentioned in our introduction, this feature is very important in order to control the optical characteristics 


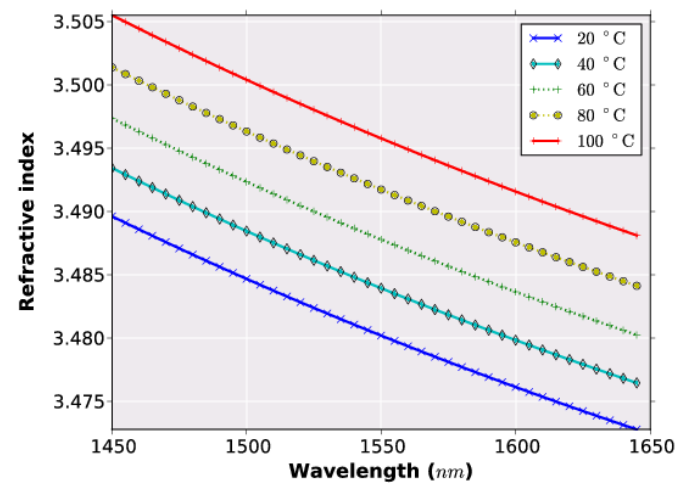

(a) Silicon

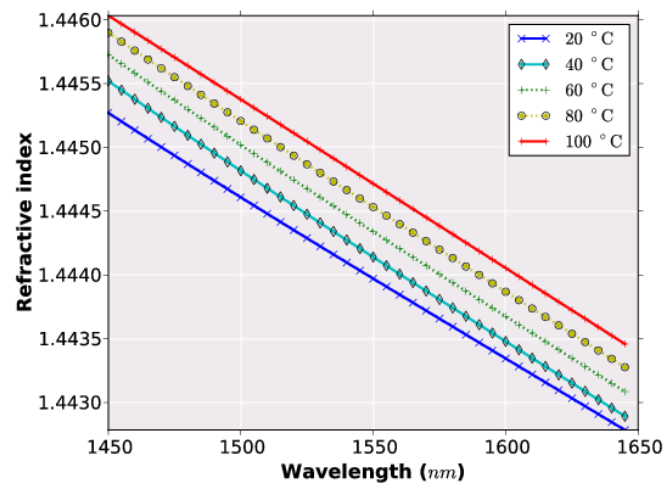

(b) Silicon Dioxide

Figure 4.2: Material dispersion with Temperature dependence for $\mathrm{Si}$ (a) and $\mathrm{SiO} 2$ (b)

of a dielectric waveguide, in particular for resonance tuning control.

With the material dispersion models, next step was to calculate the propagating mode characteristics of a dielectric waveguide by a process known as optical mode solving.

The method employed to calculate an eigenmode of a dielectric waveguide is a frequency domain mode solving technique based on the plane-wave basis expansion method [87, 38]. The plane-wave basis technique was applied in the analysis of silicon-oninsulator (SOI) waveguides with demonstrated and acceptable accuracy compared to experimental data [88]. The plane-wave basis technique is implemented as open source software named MIT Photonic Bands (MPB).

Specifically, the objective of mode solving is to obtain the effective index, group index and mode profile of a straight SOI waveguide. Because MPB's plane wave expansion is known as a technique to calculate band structures of dielectric waveguides [89], this tool was programmed to solve iteratively for a specific optical frequency $(\omega)$ in order to obtain the propagation constant $(\beta)$. From there, the effective index is extracted from $n_{\text {eff }}=c \cdot \beta / \omega$, where $c$ is the speed of light in vacuum.

The transverse mode profiles for quasi-TE and quasi-TM obtained from MPB are shown in Fig. 4.3(a) and 4.3(b) respectively. The simulation used an uniform grid with a 
size of $2 \mathrm{~nm}$, which allows for acceptable accuracy at reasonable computation times. On those graphs, the dark line represents a contour of the SOI waveguide, and the colored contours represent the E-field mode intensity. The effective index was calculated at a specific wavelength of $1550 \mathrm{~nm}$ and temperature of $22{ }^{\circ} \mathrm{C}$ (room temperature). The values obtained were 2.4677 for quasi-TE and 1.9634 for quasi-TM. Also, the mode profile of Fig. 4.3(a) shows a high confinement of an E-field in the waveguide core for quasi-TE single mode propagation. Compared to Fig. 4.3(b), a quasi-TM field profile shows high intensity on the top and bottom core-cladding boundaries, which suggest this mode will experience higher propagation loss due to its poor optical confinement. In summary, this cross-section is optimized for quasi-TE mode propagation as demonstrated in other studies [10].

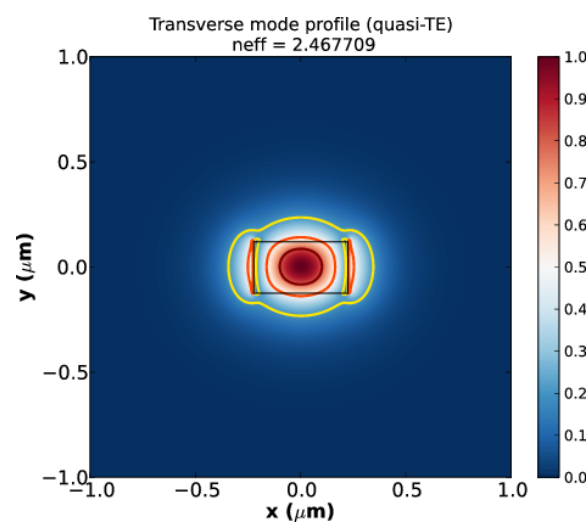

(a) quasi-TE mode $-E_{x}$

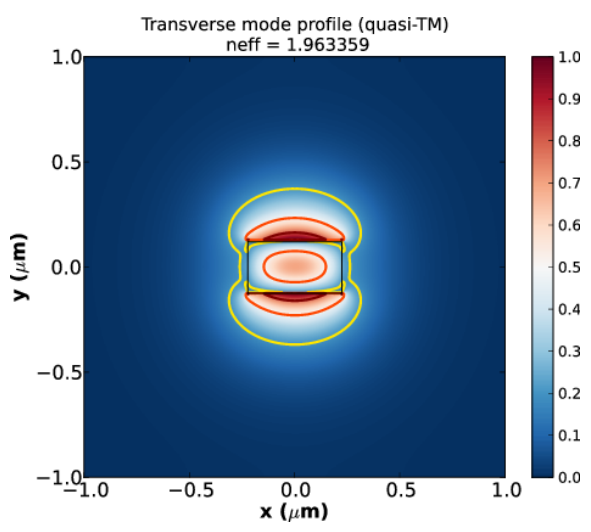

(b) quasi-TM mode - $E_{y}$

Figure 4.3: E-field mode profiles for quasi-TE (a) and quasi-TM (b) polarizations.

The simulation results of this straight waveguide using plane wave expansion method (MPB) were verified using a correlation based Vectorial 3D Beam Propagation Method [90] and a Vectorial Finite Difference Mode Solver [91]. The maximum relative difference between MPB and the finite difference mode solver was less than $0.08 \%$ and compared to Beam Propagation method the difference was less than $0.39 \%$. Therefore, the plane wave method can be employed in modal analysis of SOI waveguides because 


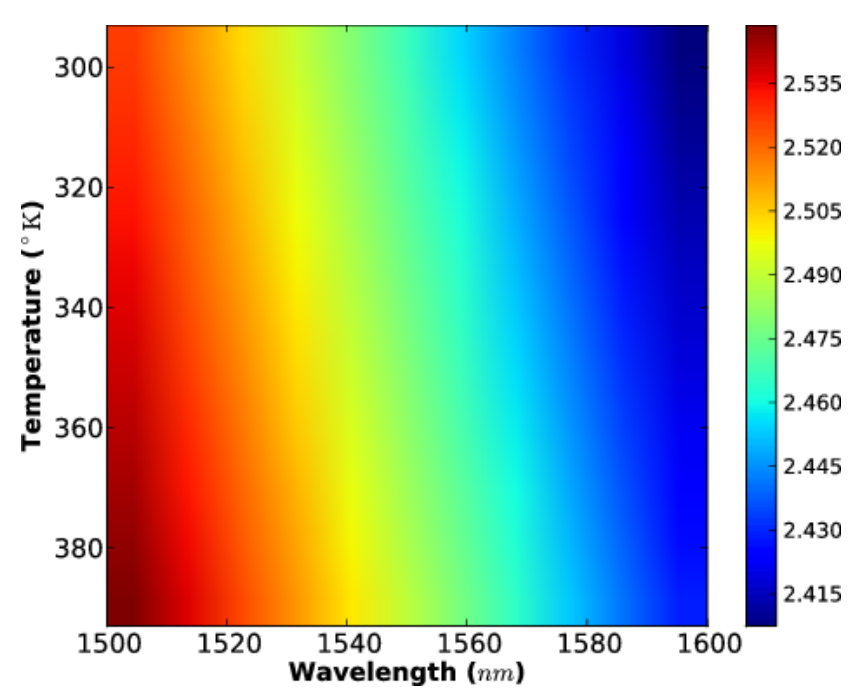

Figure 4.4: Effective index of a straight SOI waveguide (quasi-TE fundamental mode) of $450 \times 250 \mathrm{~nm}^{2}$ cross-section as a function of wavelength and temperature.

of its acceptable accuracy, with the added benefit that its code is accessible at low cost, customizable and easily to be used with Python [92] programming scripts as was done in this work for numerical data processing $[93,94]$ and presentation.

Because, temperature dependent material dispersion models for $\mathrm{Si}$ and $\mathrm{SiO} 2$ were already defined, it is possible to find the temperature dependence and waveguide dispersion of a SOI straight waveguide. Using MPB, we calculated the effective index as a function of wavelength and temperature for a SOI structure of $450 \times 250 \mathrm{~nm}^{2}$ cross-section. The effective index as a function of wavelength and temperature is shown in Fig. 4.4. We considered a temperature range from $20{ }^{\circ} \mathrm{C}$ to $100{ }^{\circ} \mathrm{C}$ and wavelength region including the C-band. As expected, the effective index of the waveguide is highly sensitive to temperature, due to silicon high thermo-optic coefficient. Apparently, the effective index relation on both wavelength and temperature looks linear, but rather it has small non-linear dispersion components.

From numerical calculations of the waveguide dispersion and temperature effects, it is possible to fit the resulting numerical data into a quadratic function of two variables. 


$$
\begin{array}{ll}
\lambda_{0}=1550.0 \mathrm{~nm} & T_{0}=343^{\circ} \mathrm{K} \\
\sigma_{\lambda}=1.0 \mathrm{~nm} & \sigma_{T}=1.0^{\circ} \mathrm{K} \\
n_{0}=2.47787548 & n_{1}=2.18504555 \cdot 10^{-4} \\
n_{2}=1.37300146 \cdot 10^{-7} & n_{3}=-1.19512675 \\
n_{4}=-4.86809255 \cdot 10^{-5} & n_{5}=5.09552492 \cdot 10^{-8} \\
n_{6}=-1.10444612 \cdot 10^{-1} & n_{7}=-4.90696704 \cdot 10^{-5} \\
n_{8}=-1.62106413 \cdot 10^{-7} & \text { mean error } f i t<4.1 \cdot 10^{-6}
\end{array}
$$

Table 4.2: Fitted parameters for effective index of a straight SOI waveguide (quasi-TE fundamental mode).

Based on the procedure explained in [86], a second order Taylor expansion approximation was applied to the effective index calculated data shown in Fig. 4.4. The curve fit model is expressed as [86]:

$$
\begin{gathered}
n_{\text {eff }}^{S W}(\lambda, T)=N_{o}(T)+N_{1}(T)\left(\frac{\lambda-\lambda_{0}}{\sigma_{\lambda}}\right)+N_{2}(T)\left(\frac{\lambda-\lambda_{0}}{\sigma_{\lambda}}\right)^{2} \\
\left\{\begin{array}{l}
N_{0}(T)=n_{0}+n_{1}\left(\frac{T-T_{0}}{\sigma_{T}}\right)+n_{2}\left(\frac{T-T_{0}}{\sigma_{T}}\right)^{2} \\
N_{1}(T)=n_{3}+n_{4}\left(\frac{T-T_{0}}{\sigma_{T}}\right)+n_{5}\left(\frac{T-T_{0}}{\sigma_{T}}\right)^{2} \\
N_{2}(T)=n_{6}+n_{7}\left(\frac{T-T_{0}}{\sigma_{T}}\right)+n_{8}\left(\frac{T-T_{0}}{\sigma_{T}}\right)^{2}
\end{array}\right.
\end{gathered}
$$

The variable $n_{e f f}^{S W}$ represents the curve fit approximation of the effective index of a straight waveguide $\left(450 \times 250 \mathrm{~nm}^{2}\right)$, this approximation is centered at a wavelength of $1550 \mathrm{~nm}$ and a temperature of $343{ }^{\circ} \mathrm{K}$. The rest of the curve fit parameters are shown in Table 4.2. The maximum relative error of the curve fit is less than $5.2 \times 10^{-4} \%$. With this model, it opens up the possibility to employ it on further calculations of group index and to use it in modeling the thermal tuning of a ring resonator filter.

The effective index model for a straight waveguide can also be extended to a curved or bent waveguide. The procedure to obtain an approximation of the waveguide dispersion for a ring waveguide will be explained next. 


\subsubsection{Micro-ring resonator radius}

As mentioned in Chapter 2 , the free spectral range (FSR), which denotes the separation between resonances in a micro-ring resonator, is inversely proportional to the resonator length. This length is controlled by the ring radius, thus reducing the radius will increase the FSR. Having large FSR could allow many spectral channels to be processed by an array of single ring resonators and to avoid channel overlaps. The effect by reducing the ring radius is two-fold, first, the bending loss is increased, which impacts directly into the filter Quality factor by reducing it. The second effect is due to technology limitations, as the ring size reduces, the device is more likely to suffer from lateral surface roughness, which introduces scattering losses. This type of loss is the cause of a further reduction of the Q-factor, which can be noticed by an increase of the resonance bandwidth (FWHM). For applications in telecommunications where more bandwidth is necessary for data transmission, this increase of bandwidth is welcomed; however for purposes of sensor interrogation, a narrow line-width is desirable because a more selective filter allows sampling the sensor spectrum without spectral broadening effects.

Examples of designs published in literature are an ultra-compact ring resonator of 1.5 $\mu m$ with a $\mathrm{Q}=3100$ and FSR of $52 \mathrm{~nm}$ reported in [34]. A slightly small resonator of 2.0 $\mu m$ radius offered a Q of approx 6700 with an FSR of $47 \mathrm{~nm}$ [8]. In another research a ring resonator of $5 \mu \mathrm{m}$ had $18 \mathrm{~nm}$ of FSR and FWHM of 0.1 to $0.2 \mathrm{~nm}$ [3].

In this research, two radii were chosen: 2.0 and $5.0 \mu \mathrm{m}$ both based on previous experience from FIU's Nanophotonics Research group. Those ring sizes allow the possibility to have adequate resonance spectrum separation and potential of high integration.

Similar to a straight waveguide, it is necessary to account for the dispersion of the guided mode of a bent waveguide, which forms the ring structure. The effective index of the bent waveguide is important because it can be applied to estimate the transmission spectrum of a ring resonator [86]. 


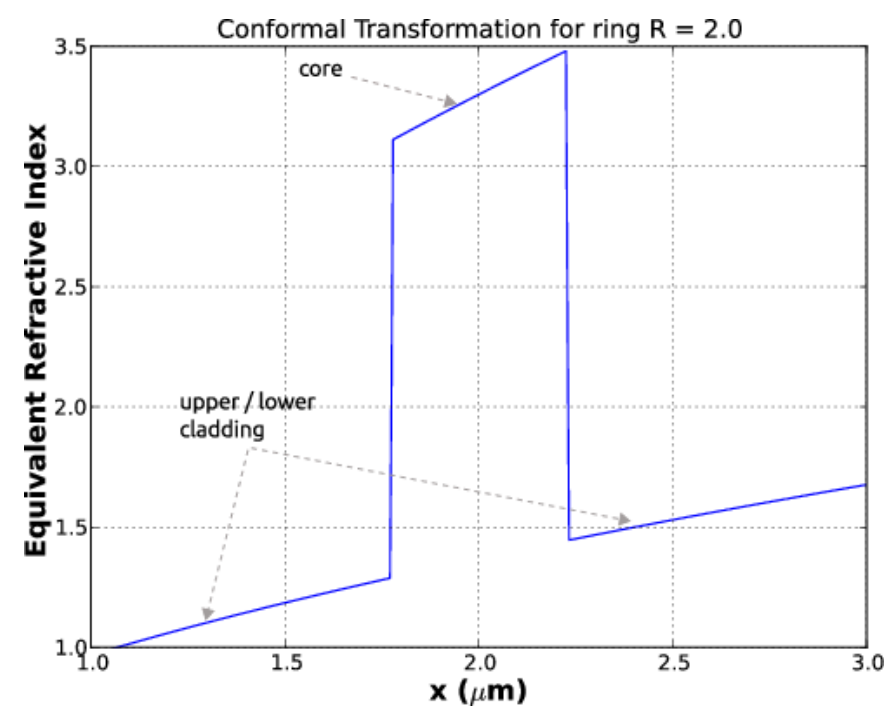

Figure 4.5: Equivalent index profile of a $2 \mu \mathrm{m}$ ring radius SOI channel waveguide of 450 $\mathrm{nm}$ x $250 \mathrm{~nm}$ cross-section after applying conformal transformation.

To solve the modes of a bent waveguide, this study will apply a conformal transformation of the ring geometry and index of refraction $[86,95]$. The conformal transformation is an approximate way to quantify the dispersion of a curved waveguide however it is not strictly rigorous. It applies only to the cross-section plane of the ring waveguide.

The conformal transformation method converts the radial $(r)$ and angular direction of the ring into two new variables: $u=R_{e} \cdot \ln (r / R e)$ and $v=2 \pi R_{e}$. To apply this transformation, a radial region is defined as $R_{\text {out }}+x_{0}+w<r<R_{\text {out }}+x_{0}$, where $R_{\text {out }}$ is the outer rim radius, the ring width is denoted as $w$ and $x_{0}$ represents the lateral spacing surrounding the ring waveguide. The way this transformation works is to convert a bent waveguide into an "equivalent" straight waveguide. After this transformation, the refractive index of the core and cladding will be modified as defined by variables $u$ and $v$. A demonstration of how the refractive index profiles are modified is shown in Fig. 4.5. 
The index change is expressed in the equation below, where $R_{e}$ was arbitrarily chosen in order to make $u=0$ at $R_{e}=R_{\text {out }}$.

$$
n_{e q}=n_{b e n t} \cdot \exp u / R_{e}
$$

Once we applied a conformal transformation, next step was to perform a mode solving of the equivalent straight waveguide. The plane wave expansion method (MPB) tool was again used for this particular task. This simulation applied a mesh geometry with uniform grid spacing of $2.0 \mathrm{~nm}$ for the dielectric structures. In this step, the objective was to obtain the effective and group index at multiple values of wavelength, temperature and bend radii.

Following [86] an approximated expression of the effective index for a bent waveguide can be obtained. The effect of bending is modeled independently from the effects on temperature and wavelength. In other words:

$$
n_{e f f}^{\text {ring }}(\lambda, T, R)=n_{e f f}^{S W}(\lambda, T) \cdot f(R)
$$

Here, the previously modeled effective index of a SOI straight waveguide $\left(n_{e f f}^{S W}\right)$ is considered together with a new fitting function defined as [86]:

$$
f(R)=\frac{k_{1}\left(1-\exp \left[-k_{2}\left(R+R_{0}\right)\right]\right)+k_{3}\left(1-\exp \left[-k_{4}\left(R+R_{0}\right)\right]\right)}{k_{1}+k_{3}}
$$

where $k_{1} \cdots k_{4}$ are parameters arising from the curve fit to numerical data and $R_{0}$ is radius parameter for curve adjustment to numerical data.

After mode solving, the numerical results of the effective index of the ring were fitted by a three dimensional function based on Eq. 4.3. Results of the effective index curvature dependence at wavelengths from 1500 to $1600 \mathrm{~nm}$ and temperatures from $20{ }^{\circ} \mathrm{C}$ to 120 ${ }^{\circ} \mathrm{C}$ are shown in Fig. 4.6. The inset of that figure shows the relative modification of the 


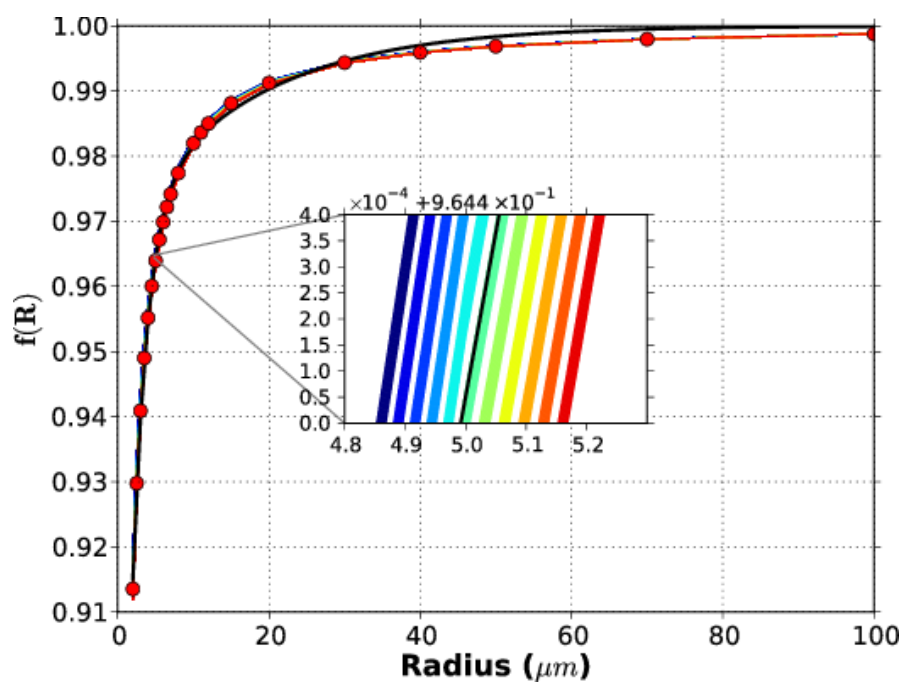

Figure 4.6: Curvature function $f(R)$ fit to numerical data of quasi-TE effective index of bent SOI waveguide. Inset represents the change in effective index as function of wavelength and temperature, colors represent same wavelength.

$$
\begin{array}{ll}
R_{0}=3.46 \mu \mathrm{m} & k_{1}=5.44488759 \\
k_{2}=0.05874783 \mu \mathrm{m}^{-1} & k_{3}=136.00368704 \\
k_{4}=0.51727391 \mu \mathrm{m}^{-1} & \text { max. relative } \text { error }_{\text {fit }}<0.44 \%
\end{array}
$$

Table 4.3: Fitting parameters for curvature function $f(R)$

effective index as function of wavelength and temperature while colors represent same wavelength but at different temperatures.

In Fig. 4.6, the black line represents the fitted curvature function $f(R)$ with parameters shown in Table 4.3. The error of the curve fit formula in Eq. 4.4 is $0.44 \%$ compared to the ratio between simulated values of $n_{\text {eff }}^{\text {ring }}$ vs. $n_{\text {eff }}^{S W}$ obtained from mode solving simulations.

\subsubsection{Resonances and free spectral range}

In the previous subsection a semi-analytical model of the effective index of a bent waveguide was defined. According to Eq. 2.1 from Chapter 2, the resonance wavelength of a ring resonator depends on its radius, resonance order number and effective index. Because a ring waveguide has a large modal dispersion, this means that to find the resonance 


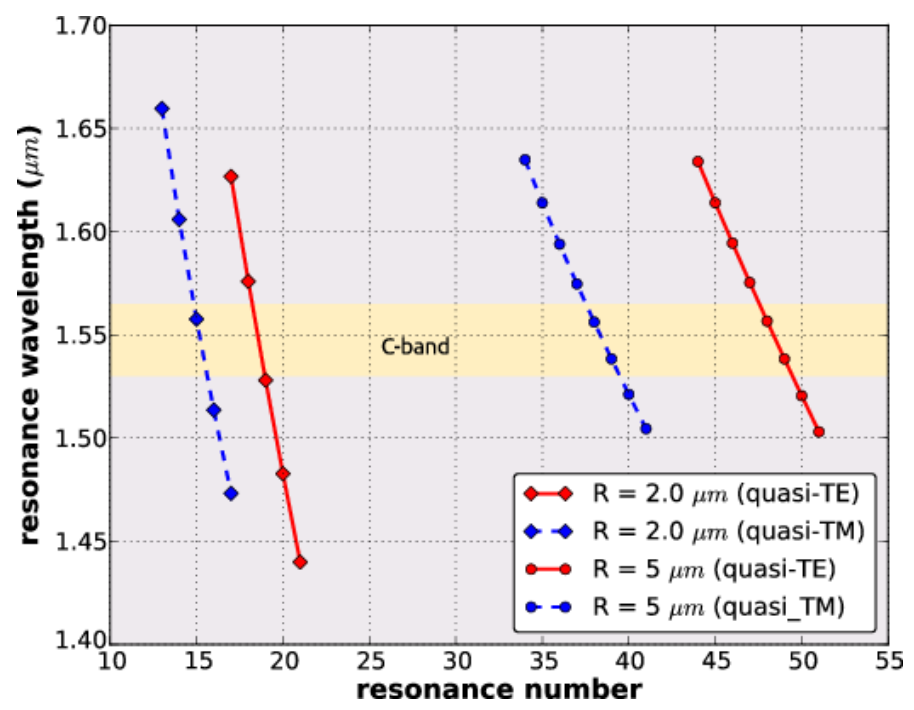

Figure 4.7: Resonance wavelengths of two MRR designs of radius $2 \mu \mathrm{m}$ and $5 \mu \mathrm{m}$ for quasi-TE and quasi-TM polarizations.

we need to solve a transcendental equation. The way to obtain a solution for a particular ring radius is by obtaining iteratively the root of the function $\lambda_{m}-\frac{2 \pi R}{m} n_{\text {eff }}^{\text {ring }}(\lambda)$. Fig. 4.7 shows the computed resonances for both MRR designs $(2 \mu \mathrm{m}$ and $5 \mu \mathrm{m})$ and for TE and TM polarizations.

Considering the quasi-TE case of a ring with $5 \mu \mathrm{m}$ radius, we have two resonances inside the C-band at $\mathrm{m}=48$ and 49 , also the average FSR is about $18.7 \mathrm{~nm}$, which is similar as reported in [22]. In addition there are quasi-TM resonances close to the quasiTE ones, on average the separation of both modes within the C-band is $0.24 \mathrm{~nm}$.

On the other hand, considering the design of a ring with $2 \mu \mathrm{m}$ radius, there is a single resonance close to the $\mathrm{C}$-band for the quasi-TE case, even considering fabrication effects like surface roughness and dimensional errors that resonance is expected to reach the Cband. In addition, this design has the advantage of theoretically having large FSRs, i.e. the average FSR for a $2 \mu m$ radius MRR is around $46.4 \mathrm{~nm}$, greater than our initial design requirement of $35 \mathrm{~nm}$. 
Large resonance separation will have the advantage of preventing any spectrum folding effects in optical sensor interrogation systems based on optical filters as was mentioned in Chapter 3.

\subsubsection{Losses in a micro-ring resonator}

An electromagnetic field propagating through an optical waveguide, such as a microring resonator, experiences losses due to several causes: scattering from sidewalls due to fabrication limitations, optical substrate leakage, material absorption loss and bend losses.

Understanding loss mechanisms is important in order to correctly design a micro-ring resonator. Intrinsic losses control the spectral characteristics of the MRR, i.e. losses affect the quality factor ( $Q$ factor), which manifest in wider or narrow spectrum line-widths and also affects the transmission magnitudes of the MRR output ports.

Of the above mentioned causes for optical loss, absorption losses in undoped silicon at room temperature are smaller than scattering and optical leakage losses [86]. The most significant loss contribution comes from scattering at sidewalls due to surface roughness during lithographic fabrication. Second in importance is the substrate leakage and bend losses, both of those can be predicted by electromagnetic simulation. However, scattering losses are difficult to model and predictions are not reliable enough, because of the different fabrication conditions and techniques. Based on reported experiments found in literature, we can estimate a range for the round-trip field loss based on similar crosssections and fabrication techniques. For example, in micro-ring resonators with $2.25 \mu \mathrm{m}$ of radius, a round trip field loss of $5 \%$ at $1550 \mathrm{~nm}$ wavelengths was achieved [32]. For a radius of $4.5 \mu \mathrm{m}$ round trip field loss coefficient was $0.5 \%$ [31]. For a radius of $5 \mu \mathrm{m}$, the round trip power loss coefficient was $0.27 \%$ [32]. Lower losses were obtained in wider ring resonators (20 $\mu \mathrm{m}$ radius) with strip waveguides reaching $0.2 \%$ or $1.9 \mathrm{~dB} / \mathrm{cm}$ [35]. 
Therefore, an estimated round trip loss range for a MRR of $2 \mu \mathrm{m}$ radius can be set from 4 to $6 \%$ and for a micro-ring of $5 \mu \mathrm{m}$ radius, the loss range lies within 0.3 to $0.5 \%$.

\subsubsection{Waveguide separation distance}

The separation distance between the incoming / outgoing straight waveguides and the ring waveguide itself is another design parameter, which is directly related to the amount of power coupling. From chapter 2, the power coupling coefficient controls the amount of energy storage measured by the Q-factor of the resonator. In addition, the Q factor is inversely related to the resonance bandwidth or FWHM. High Q-factor resonators usually show narrow resonances in their spectra.

Usually, the amount of power coupling to the resonator is calculated based on the device structure and controlled by the waveguide separation distance known also as gap distance. This problem can be treated using theoretical analysis based on coupled of modes in space [37] but the analysis turns out a bit complex due to the curved nature of the ring waveguide. Another approach is based on numerical simulations, such as the Finite Difference Time Domain (FDTD) method, a technique employed by researchers to solve Maxwell equations in high index contrast optical waveguides whose dimensions are comparable to the radiation wavelengths $[96,97,98,99,100]$.

The FDTD method divides a spatio-temporal domain into a discretized grid and simulates the evolution of the electromagnetic fields in time based on Maxwell equations [101]. A complete explanation of the method and its numerical algorithms are outside the scope of this study, however there are numerous references that treat the subject in detail $[96,101,102]$. Also, there are many commercial softwares that implement the FDTD method, such as Rsoft's Fullwave. Additionally, open source software tools, such as Meep from the Ab Initio's group at MIT [101] offer the advantage of flexibility, low 
entry cost and an ever increasing research community with published results. With those tools, we were able to integrate Meep with Silicon Photonics Computer-Aided Design libraries (IPKISS ${ }^{1}$ ) using Python scripts to allow their interaction.

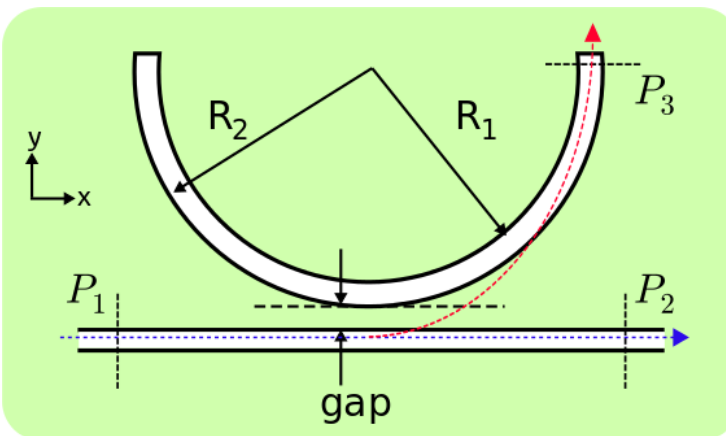

Figure 4.8: Upper view of a MRR geometric structure used for power coupling computation with 3D FDTD.

A power coupling coefficient corresponds to the amount of power coupled into the ring from an input bus waveguide. [96]. Consider the schematic of a ring resonator shown in Fig. 4.8. Due to computing limitations, half of the structure is actually implemented for 3D FDTD simulations. In that figure, three planes are denoted, plane P1 measures the power flux (Poynting vector) at the source, P3 measures the power at a quarter circumference of the ring, and P2 measures the power left at the output port of the bus waveguide.

The longitudinal flux power passing through those three planes: P1, P2 (parallel to yplane) and P3 (parallel to X-plane) are recorded at each time step of the FDTD simulation. Once the field inside the ring has passed a quarter of the circumference simulation stops and then a Fast Fourier Transform (FFT) is applied to compute the power flux at each plane as a function of frequency.

To obtain the power coupling coefficients, a "band limited" Gaussian optical pulse of $6.6 f s$ with a wavelength of $1.55 \mu \mathrm{m}$ was launched at the input port of the bus waveguide.

\footnotetext{
${ }^{1}$ A parametric design software environment for integrated photonics from Ghent University http://www.ipkiss.org/
} 
The FDTD simulation used a 3D uniform grid size of $20 \mathrm{~nm}$ with a time step of 0.0166 fs (consistent with the Courant Stability condition)[102]. Simulations were performed for two different resonator radii, e.g. $2 \mu \mathrm{m}$ and $5 \mu \mathrm{m}$. The geometric structure of the dielectric waveguide components is similar to Fig. 4.8 and the boundaries of the FDTD computing region were set to be perfectly matched layers (PML). The tool used in these simulations was MIT's Meep [101].

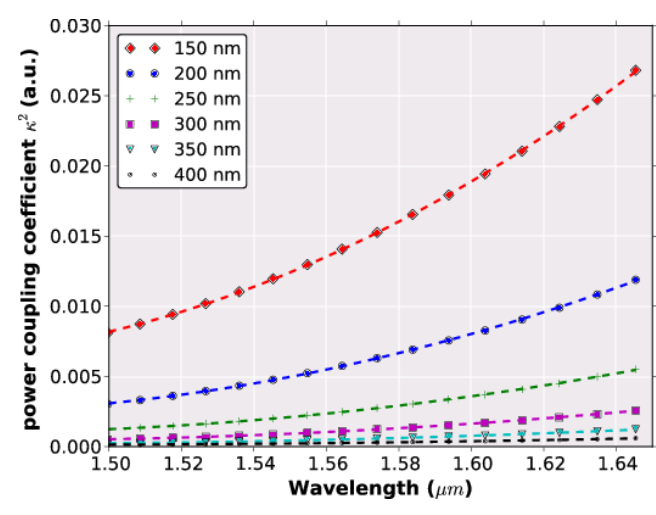

(a) $2 \mu \mathrm{m}$ radius

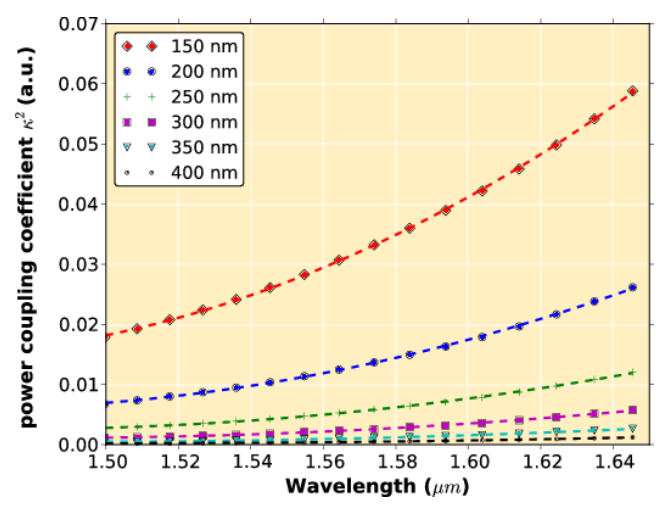

(b) $5 \mu \mathrm{m}$ radius

Figure 4.9: Power cross-coupling coefficient $\kappa^{2}$ dispersion graph for $2 \mu \mathrm{m}$ radius ring (a) and $5 \mu \mathrm{m}$ radius ring (b), for quasi-TE mode.

Fig. 4.9 shows the computed coupling efficiencies for quasi-Te polarization and for wavelengths varying from 1.5 to $1.6 \mu \mathrm{m}$. In Fig. 4.9(a), results correspond to a $2 \mu \mathrm{m}$ radius micro-ring and Fig. 4.9(b) is for the $5 \mu \mathrm{m}$ ring resonator. Considering a fixed value of gap distance, the coupling efficiency increases at higher wavelengths because at those values the modal field outside the core reaches more the ring waveguide thus enhancing the interaction with the modal field at the ring [96]. We also observe that the coupling efficiency levels increase at large micro-ring radius. The coupling efficiency for a $5 \mu \mathrm{m}$ ring is approximately twice the value compared to a $2 \mu \mathrm{m}$ ring radius. The reason is the large interaction length between the ring and bus waveguides as the total length of the ring is increased. 


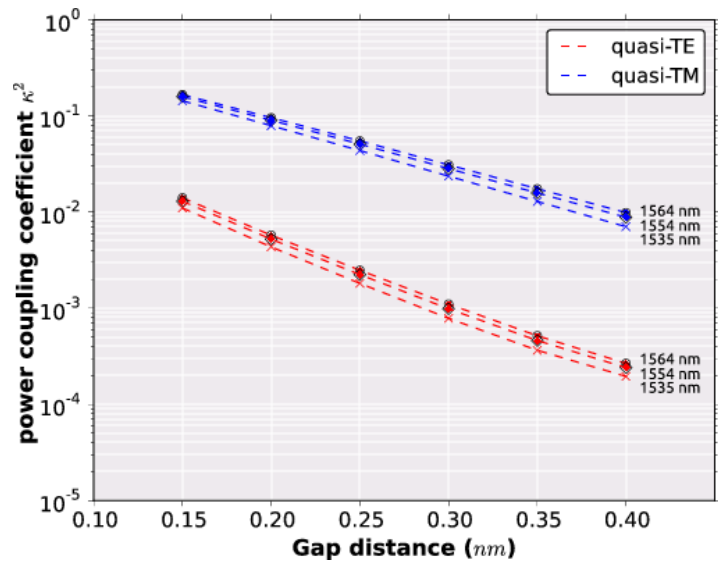

(a) $2 \mu m$ radius

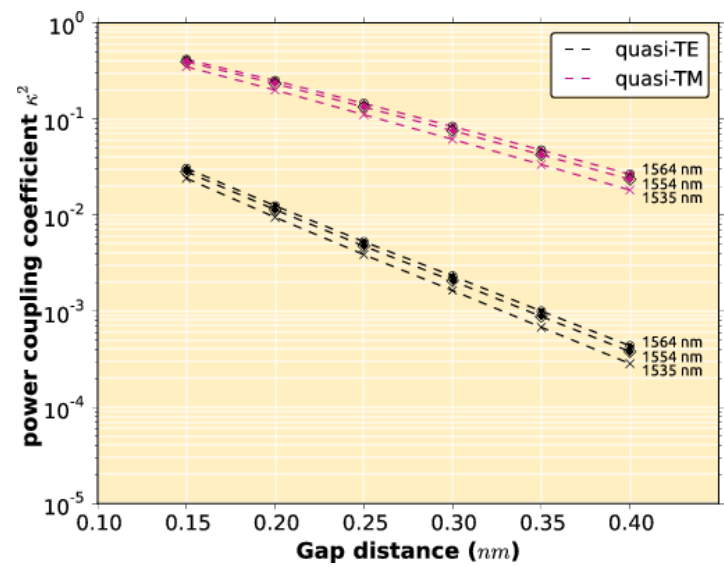

(b) $5 \mu \mathrm{m}$ radius

Figure 4.10: Power cross-coupling coefficient $\kappa^{2}$ vs. bus-ring gap distance for $2 \mu \mathrm{m}$ radius ring (a) and $5 \mu \mathrm{m}$ radius ring (b), for quasi-TE and quasi-TM modes.

Fig. 4.10 shows the computed coupling efficiency for TE and TM polarizations when the gap distance is varied from $150 \mathrm{~nm}$ to $400 \mathrm{~nm}$. As the gap distance increases, the level of coupling decreases because less modal field tails from the bus waveguide interacts with the eigenmode of the ring resonator. Again, as the ring radius increases, the efficiency also increases proportionally by comparison between Fig. 4.10(b) and 4.10(a).

The coupling efficiency curves from Figs. 4.10(a) and 4.10(b) can help us to determine an appropriate coupling gap distance for each of the ring resonator designs. However, in order to extract this information, it is necessary to have certain design targets or objectives. In specific, for a $5 \mu \mathrm{m}$ radius micro-ring, a design target is a quality factor $Q=20,000$, which means for a resonance of $1550 \mathrm{~nm}$ the line-width (FWHM) is approximately 0.08 $\mathrm{nm}$.

For a design using a ring resonator of $2 \mu \mathrm{m}$ radius, and considering a resonance of $1550 \mathrm{~nm}$ a design target could be a line-width of $0.5 \mathrm{~nm}$, which means an approximate $\mathrm{Q}$ factor of 3100 . 
To obtain the required coupling efficiency for those two micro-ring designs, we use a contour plot shown in Fig. 4.11, where we have the finesse (dotted lines) and maximum drop port efficiency (colored solid lines) as functions of a combination of field round-trip propagation loss and power coupling factor $\left(\kappa^{2}\right)$. That graph was obtained from Eqs. $2.16,2.18,2.19$.

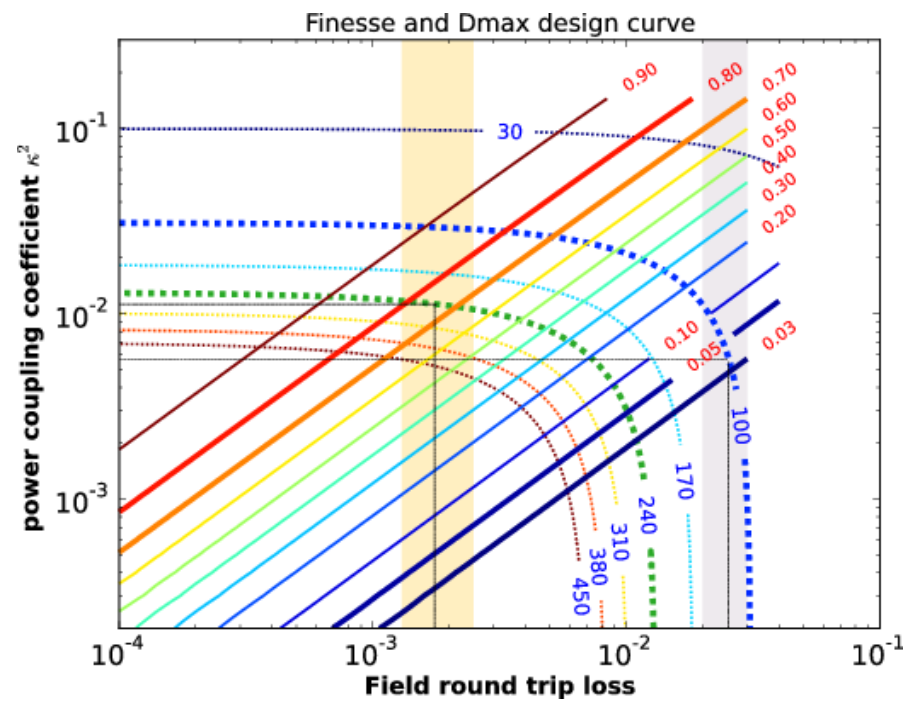

Figure 4.11: Contour plot of Finesse (dotted color lines) and Maximum Drop port Efficiency $D_{\max }$ (color full lines) as function of power coupling efficiency and Field round trip loss. Orange colored patch represents a loss region for a $5 \mu \mathrm{m}$ radius ring design, the gray patch corresponds to a ring design of $2 \mu \mathrm{m}$ radius.

As mentioned in previous sub-section we expect round-trip losses between $0.27 \%$ to $0.5 \%$ for ring resonators of $5 \mu \mathrm{m}$ radius and for $2 \mu \mathrm{m}$ radius we expect losses between $4 \%$ to $6 \%$. Fig. 4.11 shows those loss regions in gray color for $5 \mu \mathrm{m}$ radius and in orange color for the $2 \mu \mathrm{m}$ radius ring design. Now, in order to get an estimation of the coupling efficiency, we start by setting some fixed values of round trip loss for each ring designs. For example, a loss estimate of $0.35 \%$ (within the orange shaded region on Fig. 4.11) is assumed for the $5 \mu \mathrm{m}$ radius ring. Based on the target $\mathrm{Q}$ factor of 20,000 and a resonance around $1550 \mathrm{~nm}$, a finesse estimation of 240 is obtained. Looking at the contour plot (Fig. 4.11 and green dotted line), those values point to a cross-coupling power coefficient 
$\left(\kappa^{2}\right)$ of $1.12 \%$. With this estimated cross-coupling value, we can derive the coupling gap distance for the case of a $5 \mu \mathrm{m}$ radius ring resonator. By looking at Fig. 4.10(b), for $1.12 \%$ coupling the corresponding gap distance is in the vicinity of $200 \mathrm{~nm}$.

We can repeat the same procedure to the case of the $2 \mu \mathrm{m}$ ring radius design. A round trip-loss of 5\% is assumed a-priori consistent to similar designs [31]. Previously, we set a target for a $\mathrm{Q}$ factor of 3,100, this value corresponds to an approximated finesse of 96 . To accommodate for any further loss due to fabrication issues, we set a value of 100 for the finesse. Observing the contour plot in Fig. 4.11 a cross-coupling factor $\left(\kappa^{2}\right)$ of $0.57 \%$ is extracted. Therefore, from Fig. 4.10(a), the coupling gap distance can be estimated close to a value of $200 \mathrm{~nm}$. 


\section{CHAPTER 5}

\section{DEVICE FABRICATION AND CHARACTERIZATION}

In this chapter, a description of the fabrication of a micro-ring resonator filter chip is explained. We took the design results mentioned in the previous chapter and applied them in two chip designs. This research was focused in one particular chip design that was deemed very useful in the implementation of a wavelength interrogator. After fabrication, we selected two micro-ring resonator filters in order to be characterized in terms of their wavelength or spectrum response and their respective thermo-optical tunability. In this chapter, a complete description of the characterization methodology and processing analysis was presented. This helped us to extract key device parameters that will prove their importance later on when those devices are applied in a sensor processing application.

\subsection{Fabrication}

Two silicon photonic chips were fabricated using standard SOI (silicon over insulator) wafer and direct E-beam lithography. The initial silicon fabrication steps were done by Dr. Xuan Wang at Cornell NanoScale Facility, those chips were further processed by the author and other collaborators from CTI Renato Archer.

One chip design was designated as "multi-laser". The purpose of that design was to extend a previous research done by $\mathrm{X}$. Wang and R. Panepucci where a ring resonator was used to demonstrate a tunable optical fiber laser [5]. With that new design it may be possible to implement a tunable multi-wavelength optical fiber laser. That chip contained single micro-ring devices that were designed for this project. A CAD diagram of the multi-laser chip design is shown in Figure 5.1.

Another chip design was designated as "multi-ring". In that design we had two and three serially coupled ring resonators. The purpose of that design was to demonstrate tunability of a double and triple micro-ring resonator filters and the applicability of the 


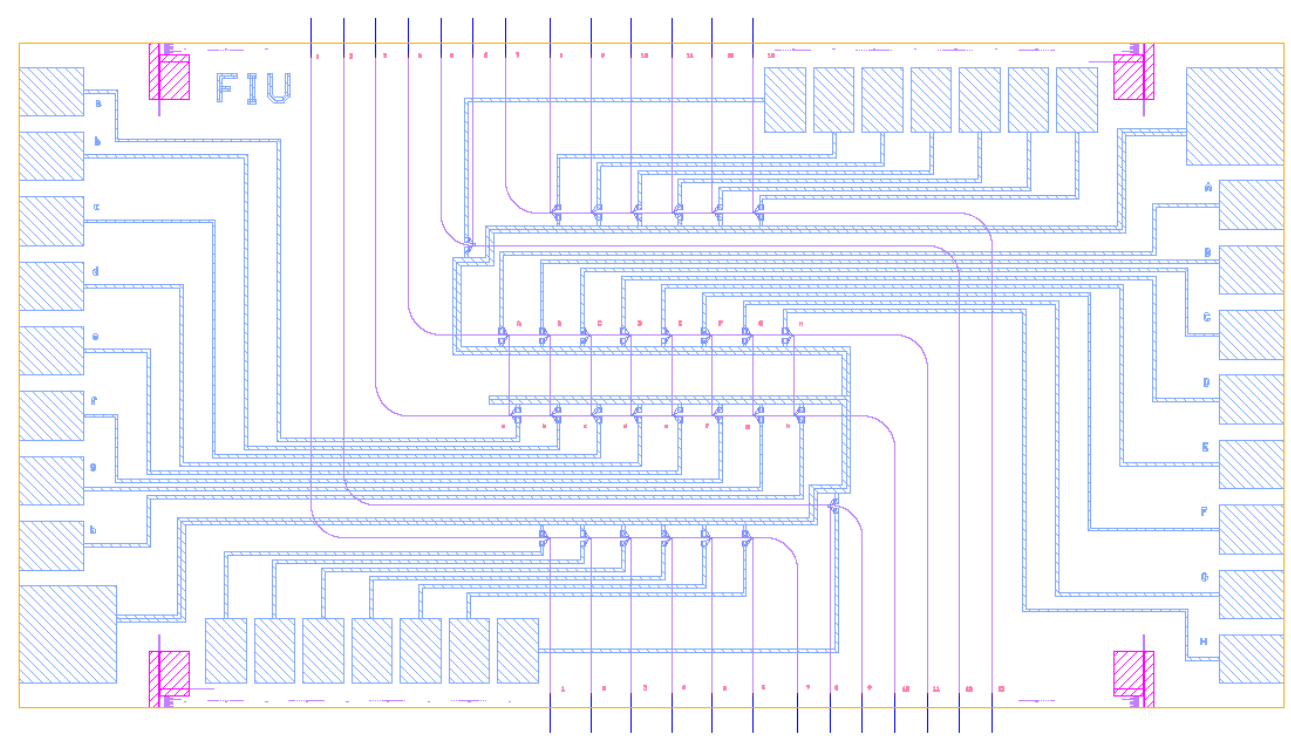

Figure 5.1: CAD diagram of designed chip.

Vernier effect in order to increase the free-spectral-range of the optical response of those filters.

Both designs employed a SOI wafer with a $250 \mathrm{~nm}$ silicon layer on $3 \mu \mathrm{m}$ of buried oxide. A cross-section diagram of the waveguides and materials are shown in Figure 5.2. The silicon waveguides were designed to have $450 \mathrm{~nm}$ of width (w), $250 \mathrm{~nm}$ of height (h) and the gap distance (g) between the straight and ring waveguides was set to $200 \mathrm{~nm}$. The optical waveguide patterns were defined by e-beam lithography over a negative photo-resist with a mask. After exposure and developing, a dry etch (RIE) was applied to the chip in order to remove any non patterned area. A low stress silicon nitride (Si3N4) film layer of $50 \mathrm{~nm}$ was deposited using low pressure chemical vapor deposition (LPECVD) so it can protect the silicon waveguides if prospective future developments were needed. Finally a one micrometer thick layer of silicon dioxide was deposited using plasma-enhanced chemical vapor deposition process (PECVD). Up to this point the silicon fabrication step was completed by Dr. Wang. After that, those chips needed further 


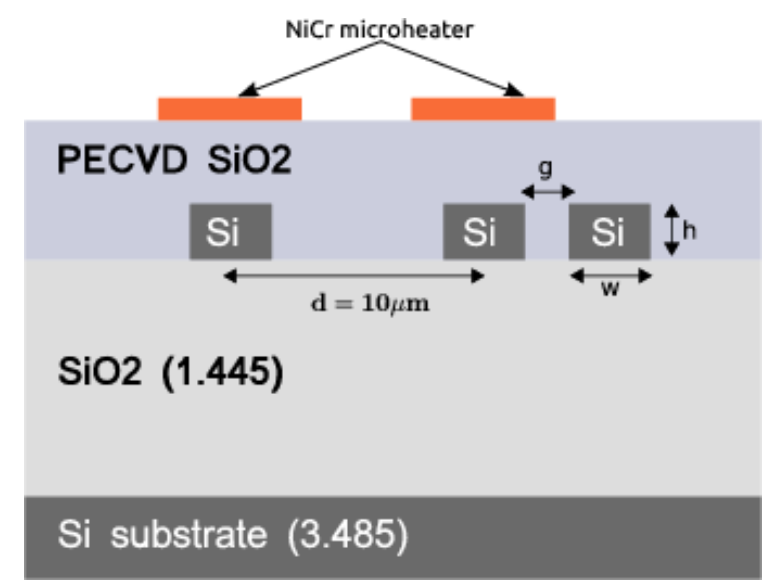

Figure 5.2: Lithography cross-section.

\begin{tabular}{ccc}
\hline Fabrication parameter & \multicolumn{2}{c}{ Values } \\
\hline Waveguide cross section & width & height \\
Coupling region gap distance & $450 \mathrm{~nm}$ & $250 \mathrm{~nm}$ \\
Radius & \multicolumn{2}{c}{$200 \mathrm{~nm}$} \\
& \multicolumn{2}{c}{$5 \mu \mathrm{m}$} \\
Heaters & Material & Thickness \\
Pad contacts & Nichrome & $100 \mathrm{~nm}$ \\
& Aluminum & $300 \mathrm{~nm}$ \\
\hline
\end{tabular}

Table 5.1: Fabrication parameter design details for a SOI micro-ring resonator.

work, such as dicing and polishing the chip facets and fabrication of microheaters.

The main fabrication parameters for the multi-laser chip design are shown in table 5.1. On the top of the multi-laser chip design, metallic micro heaters were fabricated using mask aligned UV photolitography over positive photo-resist.

Following the photo-lithograph, a $100 \mathrm{~nm}$ Nichrome film layer was deposited using metal evaporation with a lift-off. A second step was needed in order to fabricate the contact pads and feed-lines, which was done by another UV photolithography followed by Ti/Au/Ti deposition (10/270/10 nm) with lift-off. Figure 5.3 show an image taken from an optical microscope of one device in a multi-laser chip (ML2). 


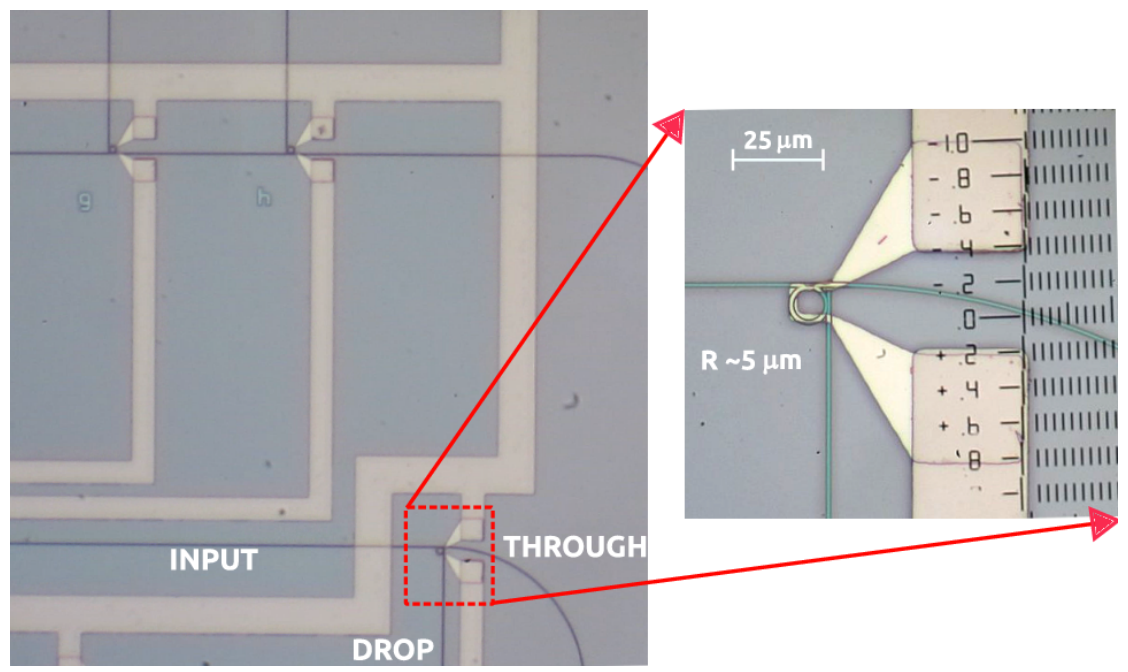

Figure 5.3: Micrograph image of a single ring resonator with a micro heater on top.

The heaters were designed with a rectangular shape because it was deemed less complex to fabricate due to resolution limitations of the UV lithography machines. Although, a circular shape would have been desirable in order to optimize the heating/power performance of the micro heaters. Because of those limitations, the micro heater shapes in this design were a bit rough.

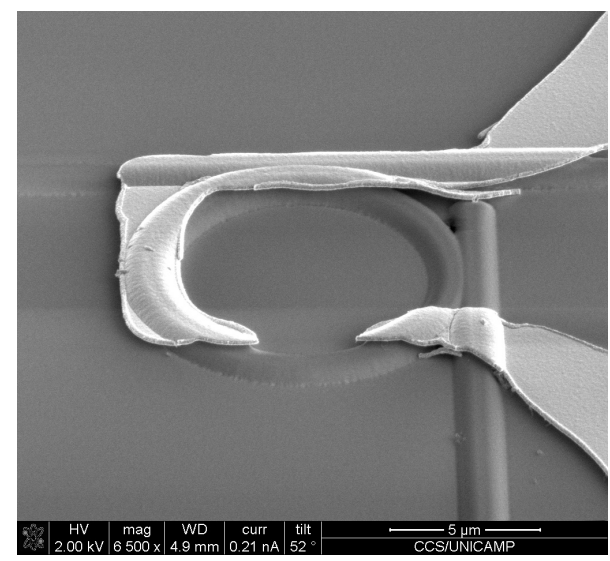

(a)

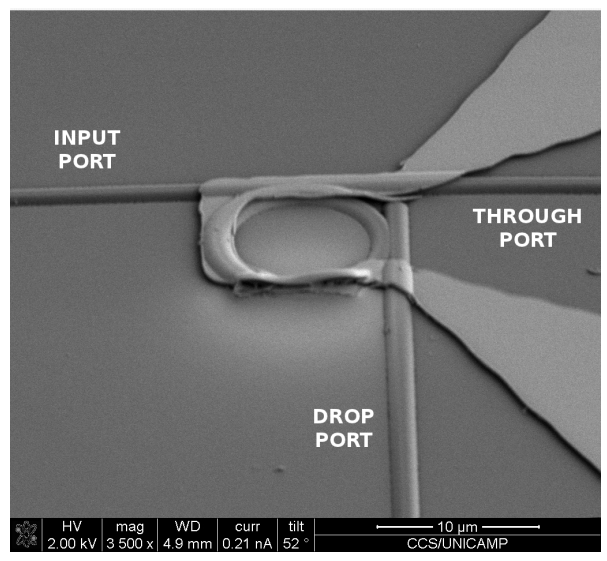

(b)

Figure 5.4: (a) Scanning Electron Microscopy (SEM) image of a device showing a break in a Nichrome micro heater. (b) Image of the same micro heater after 'fixing' it using a Focused Ion Beam (FIB) machine that injected Pt material to close the break. 
In Figure 5.4(a) one clearly observe a break in the micro heater probably due after lift-off process that followed from metal evaporation deposition. We tried to fix this issue by employing a Focused Ion Beam (FIB) machine from the Center of Semiconductor Components (CCS - UNICAMP). ${ }^{1}$ With the FIB, one can create film patterns without the need of photolitography masks. For this project we applied the FIB for (Platinum) metal deposition and fix the gap on those micro heaters. Figure 5.4(a) shows an electron microscopy image of the damaged heater, before using the FIB. Figure 5.4(b) shows the result after Pt deposition where a trace was created to bridge the gap. This process was repeated on other damaged elements with successful results. Four broken micro heaters were fixed on that chip, which allowed us to test micro-ring device functionality as will be explained later in this chapter.

\subsection{Optical characterization techniques}

The standard method to characterize an integrated optical device includes measuring the device's spectrum response. The level of spectral detail required determines, which technique is employed. One of those techniques is based on measuring the wavelength response using a spectrometer or optical spectrum analyzer (OSA) and a broadband light source coupled into the input of the optical device. One drawback of this technique is the resolution of the spectrometers lies around $10 \mathrm{pm}$, if the resonator has very narrow resonances (e.g. full-width-at-half-maximum) the spectrometer resolution will be very small. For high resolution measurements, a technique based on using a tunable laser with a photo-detector overcomes partially the limitations of typical OSA measurements, however, the measurement time will depend on how fast the laser scans over a wavelength range.

\footnotetext{
${ }^{1}$ This work was possible thanks to the help and assistance of Dr. Alfredo Rodrigues from CCS who operated the FIB system.
} 


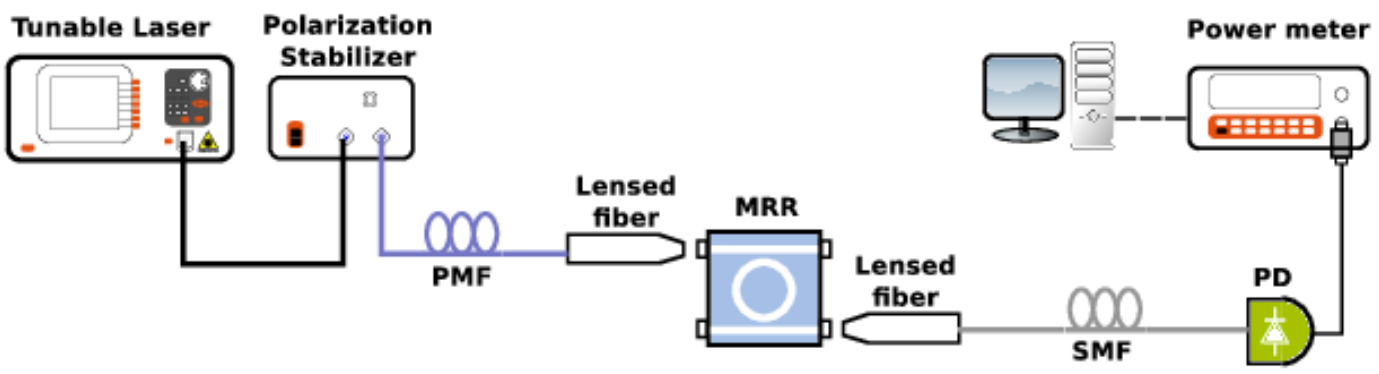

Figure 5.5: Standard measurement setup for micro-ring resonator characterization.

On Figure 5.5 a high resolution measurement setup to characterize a silicon microring resonator filter is shown. To obtain spectral resolution of $1 \mathrm{pm}$, a tunable laser is employed to scan a wavelength region of interest (usually the C-band). Light coming out the laser is polarized therefore its polarization state should be controlled along the light path with a polarization controller stabilizer. To couple light into the input of the optical device under test, we employed a polarization maintaining fiber (PMF) terminated by a lensed (or tapered) fiber. At the output port of the device under test another lensed fiber is aligned to collect light from it, and it is terminated into a calibrated photo-detector to measure any transmitted power from the laser at each wavelength step. All measurement equipment were connected to a computer by a GPIB interface. The measurement process was automated and data can be recorded by an instrumentation and data acquisition software, such as Labview.

Due to the unavailability of certain equipment, an optical characterization employing an OSA (Agilent 86146B) was chosen. This measurement equipment consists in a monochromator and is calibrated in such a way that if a wide-band light source is connected to it then the display of the equipment will appear flat along its wavelength range. When our device output is connected to an OSA any attenuation of light as a function of wavelength is recorded. Typically, an OSA wavelength resolution is between 10 to 20 pm. Therefore, an optical characterization with an OSA only enables wide spectral range measurements at the price of average resolution and slow data acquisition speed. 


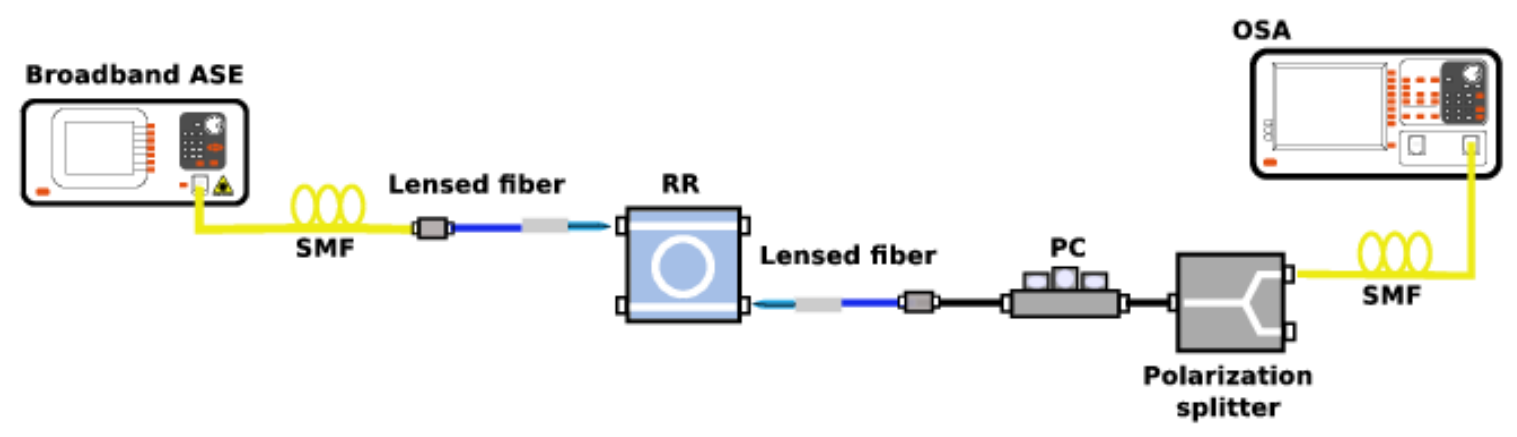

Figure 5.6: Schematic diagram for Optical characterization (spectrum measurement) of silicon photonic chips using an Optical Spectrum Analyzer (OSA).

A schematic diagram of the measurement setup is shown in Figure 5.6. In this setup, a broadband source (Erbium Doped Fiber Amplifier - IPG Photonics EAD-500-CL) was connected to our optical device by inserting light through a lensed (tapered) fiber at the input port of our device. An important thing to note is that any light source spectrum should be ideally flat along the wavelength region of interest. At the output port of our device, light was coupled using another lensed fiber. In order to select one polarization mode from the device response, we used a manual polarization controller (PC) followed by a polarization splitter (OptoLink model PBS). The output of the splitter was then connected to the OSA.

The alignment of the input and output lensed fibers is critical and was accomplished with the help of the setup shown in Figure 5.7. A mechanical XYZ precision piezoelectric stage on both sides of the sample allowed us to move each tip (input/output) of the lensed fiber until the waveguide to fiber coupling is maximized. To do that, a microscope with an objective lens allowed us to watch a magnified image of the tip of the fiber while we approach to the input/output port of the optical chip. After an initial alignment of both sides of the chip, we turned on the broadband source (EDFA as shown in Figure 5.7) and the optical power meter. Then with a piezo-electric voltage driver, we fine tune each XYZ positioning stage until a maximum of the detected power is reached. After completing the alignment process, we can proceed with any further spectral measurements. 


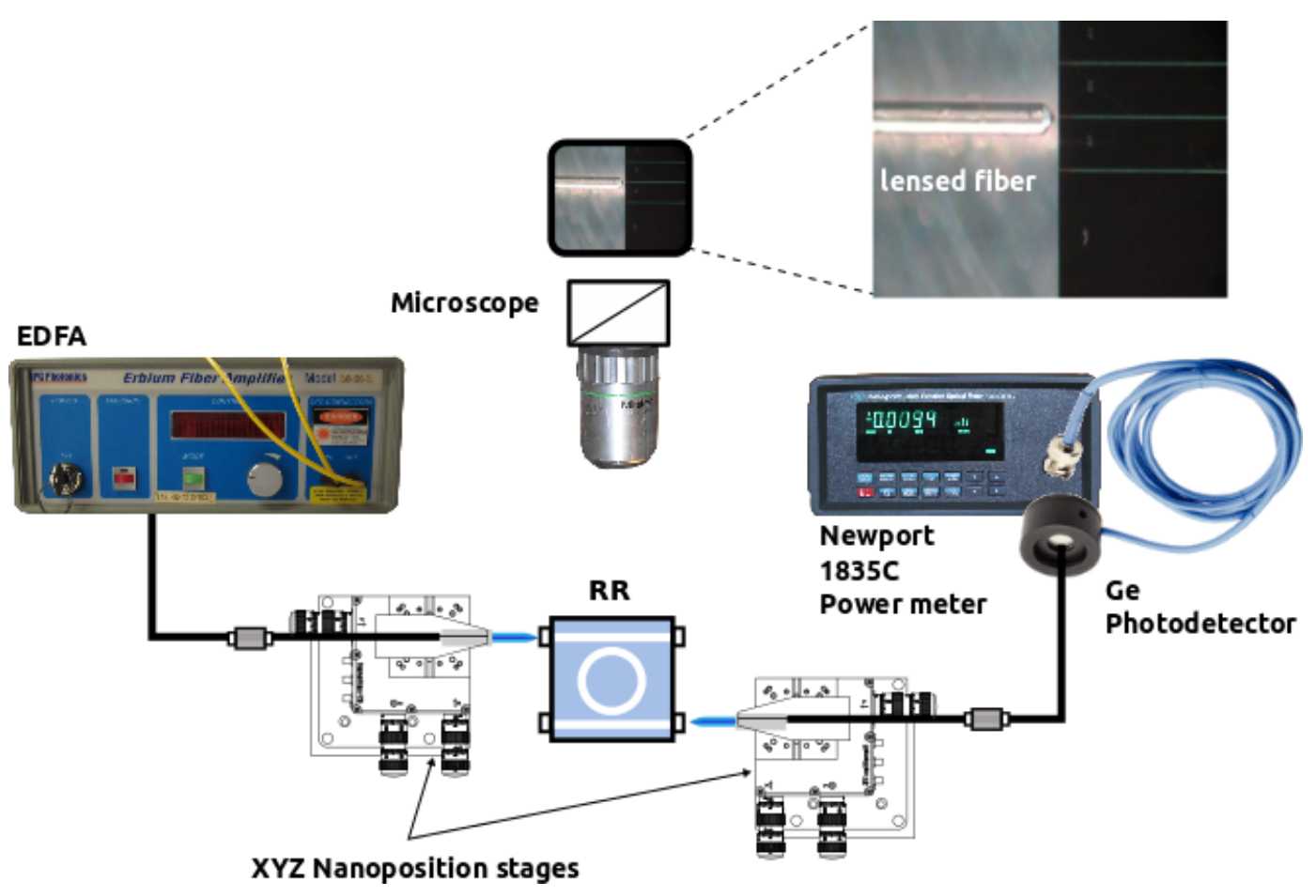

Figure 5.7: Alignment setup.

\subsection{Single micro-ring resonator measurement results and analysis}

We characterized the optical properties of our fabricated chips (named as ML1 and ML2) without applying any heating to the micro-ring devices. We chose a $5 \mu \mathrm{m}$ radius ring resonator in one chip ${ }^{2}$ and then we took the drop and through port spectra using the setup described in Figure 5.6 at room temperature.

Figure 5.8 shows the quasi-TE optical mode response of a single ring device at chip ML2 (with same designed characteristics as ML1) in a wavelength range from 1550 to $1620 \mathrm{~nm}$. The spectrum was normalized to the maximum through port transmittance power.

Clearly, the spectrum shows some fluctuations due to Fabry-Perot effect when end-fire fiber coupling is used to measure light at the facets of an optical chip [35, 8, 32]. Another

\footnotetext{
${ }^{2}$ This ring resonator was selected from other devices because of it was a single add-drop filter available from multiple WDM filters.
} 


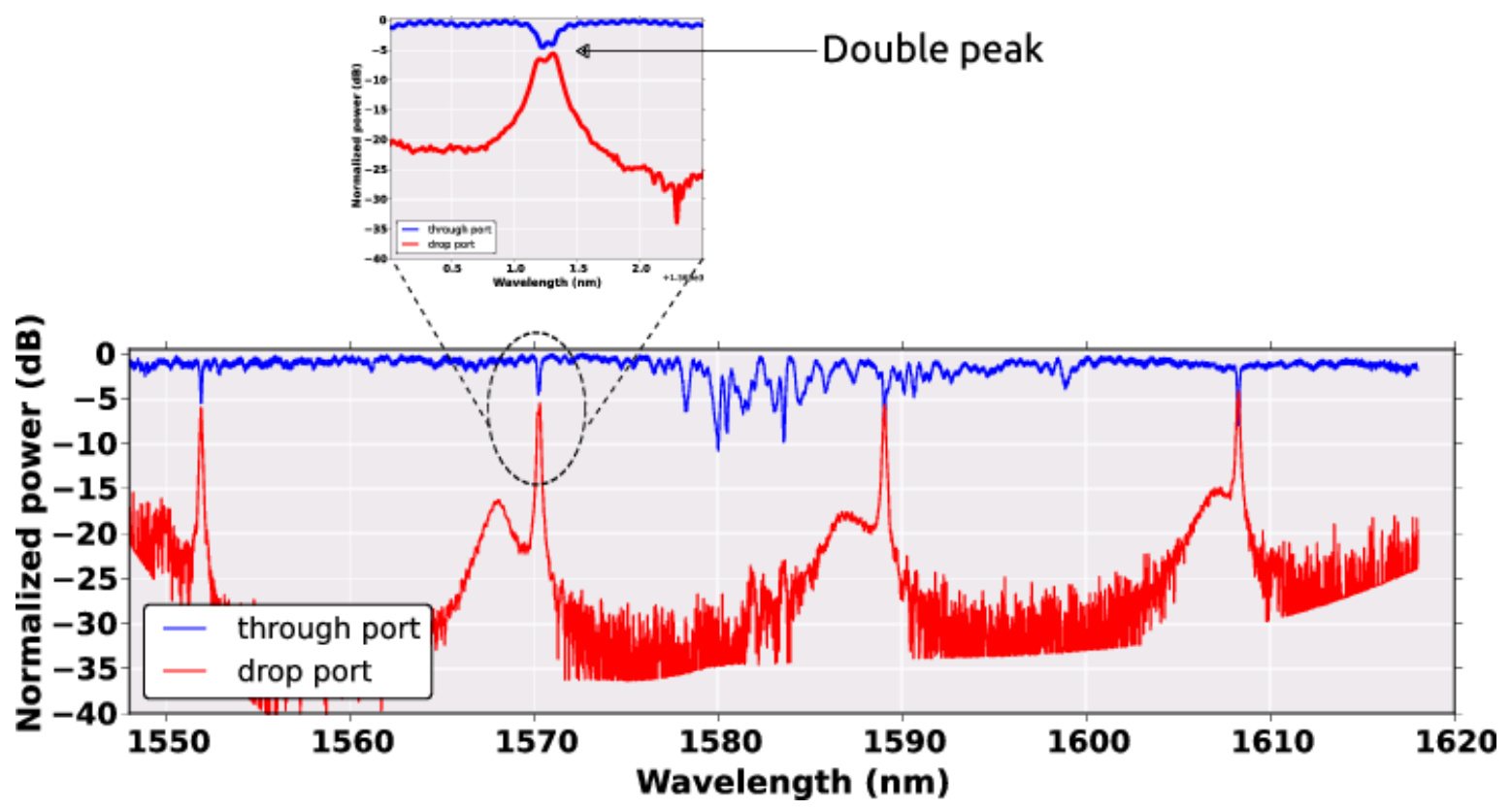

Figure 5.8: Quasi-TE spectrum response of a single micro-ring resonator of $5 \mu \mathrm{m}$ of radius and waveguide to ring gap distance of $200 \mathrm{~nm}$.

\begin{tabular}{llllr}
\hline Parameter & \multicolumn{5}{c}{ Values } \\
\hline Resonance (nm) & 1551.893 & 1570.266 & 1589.034 & 1608.261 \\
FSR (nm) & & 18.37 & 18.77 & 19.23 \\
FWHM (nm) & 0.130 & 0.195 & 0.169 & 0.188 \\
Group index & & 4.22 & 4.23 & 4.23 \\
Q factor & 11973 & 8049 & 9412 & 8538 \\
\hline
\end{tabular}

Table 5.2: Data extracted from Spectrum measurements for a single ring resonator device of $5 \mu \mathrm{m}$ radius (Quasi-TE mode).

observation from the quasi-TE optical response is the presence of double peaks at certain resonances i.e. $1570.266 \mathrm{~nm}$. Also, there are some remnant quasi-TM resonances due to the polarization control, which was done manually.

In table 5.2, we have a summary of the extracted resonant parameters taken from spectral measurements. The information about resonance and FWHM were obtained by Lorentzian curve fit over spectral data. The FSR was calculated from the extracted resonance locations, the Q factor was calculated from Eq. 2.23 and finally the group index 


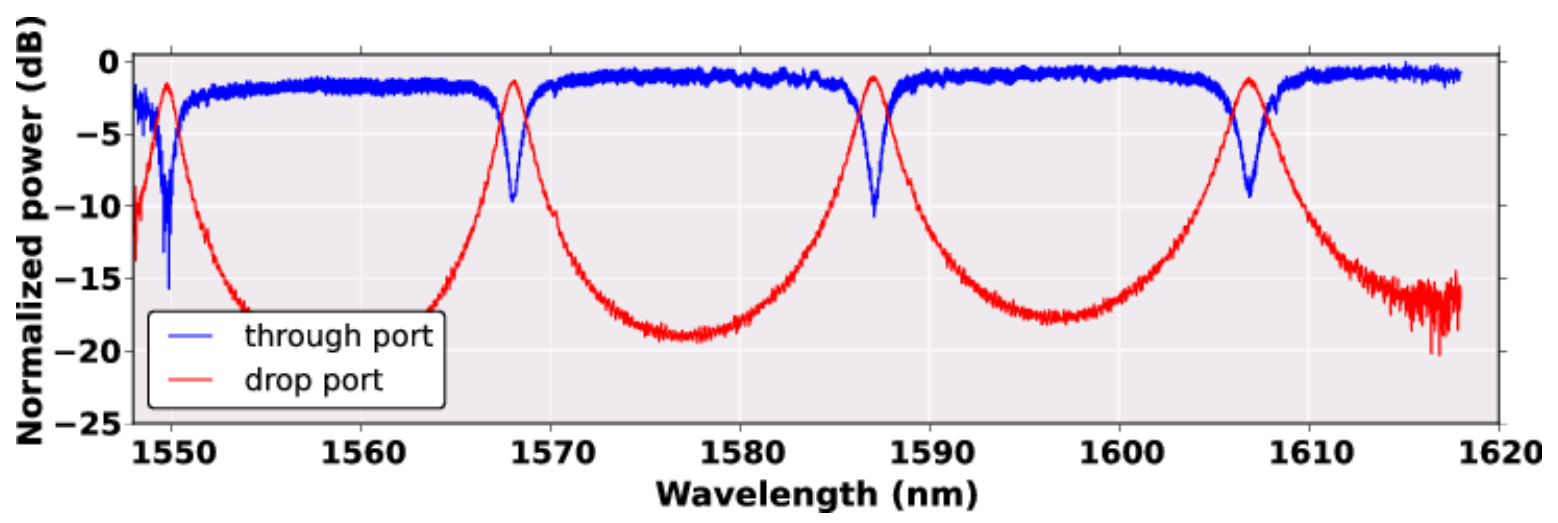

Figure 5.9: Quasi-TM spectrum response of a single micro-ring resonator of $5 \mu \mathrm{m}$ of radius and waveguide to ring gap distance of $200 \mathrm{~nm}$.

\begin{tabular}{llllr}
\hline Parameter & \multicolumn{5}{c}{ Values } \\
\hline Resonance (nm) & 1549.775 & 1568.051 & 1587.034 & 1606.863 \\
FSR (nm) & & 18.28 & 18.98 & 19.83 \\
FWHM (nm) & 1.224 & 1.464 & 1.770 & 2.176 \\
Group index & & 4.23 & 4.17 & 4.09 \\
Q factor & 1267 & 1071 & 897 & 739 \\
\hline
\end{tabular}

Table 5.3: Data extracted from Spectrum measurements for a single ring resonator device of $5 \mu \mathrm{m}$ radius (Quasi-TM mode).

were obtained from the formula derived in section 2.3.1.

The quasi-TM optical response of the same device is plotted in Figure 5.9 and the extracted parameters from those measurements are summarized in the table 5.3. As previously observed in TE mode case, still there are oscillations in the measured spectra due to the Fabry-Perot effect, which are noticeable both in through and drop port transmittances. According to tables 5.2 and 5.3, the TM and TE resonances are close $(\sim 2 \mathrm{~nm})$, which confirms the effect on the quasi-TE drop port spectrum observed in figure 5.8. This TM response shows a similar FSR as the TE response (around $18 \mathrm{~nm}$ ) but the bandwidth of the resonance is wider than the TE mode. Comparing with previous results [3] this ring resonator shows very similar free spectral range and bandwidth (FWHM), because of their similar aspect-ratio, ring radius and coupling gap distances. 


\begin{tabular}{ccccccccc}
\hline $\begin{array}{c}\text { Resonance } \\
(\mathbf{n m})\end{array}$ & $\begin{array}{c}\text { Extinction } \\
(\mathbf{d B})\end{array}$ & $\begin{array}{c}\text { Max. Drop } \\
(\mathbf{d B})\end{array}$ & $\begin{array}{c}\text { FWHM } \\
(\mathbf{n m})\end{array}$ & $\begin{array}{c}\text { Intrinsic } \\
\text { Loss }\end{array}$ & $\begin{array}{c}\text { Power } \\
\text { att. }\end{array}$ & $\begin{array}{c}\text { Power } \\
\text { coupling }\end{array}$ & $\begin{array}{c}\text { Total } \\
\mathbf{Q}\end{array}$ & $\begin{array}{c}\text { Intrinsic } \\
\mathbf{Q}\end{array}$ \\
\hline 1570.266 & 4.55 & 5.55 & 0.195 & 0.039 & 0.961 & 0.013 & 8049 & 14593 \\
1589.034 & 4.55 & 5.19 & 0.169 & 0.024 & 0.976 & 0.016 & 9412 & 20207 \\
\hline
\end{tabular}

Table 5.4: Parameters extracted from the quasi-TE mode spectrum response of a $5 \mu \mathrm{m}$ ring resonator.

We applied a model parameter extraction explained in subsection 2.3.1, in order to obtain some information about the internal loss and power coupling coefficients of our fabricated resonators. We selected one micro-ring device from chip ML2, and only considered the quasi-TE modal response. From the values summarized in Table 5.2 and with the through and drop port measured power obtained from Figure 5.8, we inserted them in the respective equations from subsection 2.3.1. A summary of the extracted parameters are shown in Table 5.4.

By observing some of the values from that table, we noticed that the power coupling coefficient of our ring is less than its internal loss value. Therefore, the maximum drop port efficiency should be lower and also the through port extinction, consistent with the theory in chapter 2 . The previous result means that our micro-ring device was not critically coupled. Additionally, the internal loss extracted is a bit large for the resonance at $1570 \mathrm{~nm}$ compared to the resonance at $1589 \mathrm{~nm}$. That is understandable due to the double-peak shape observed in the spectrum as shown in Figure 5.8. The reason for a double-peak signature could be attributed to two factors: one is a double-peak increase the measured FWHM and this effect is attributable to contra-directional coupling. This type of internal coupling occurs because inside the ring there are two opposite waveforms propagating in degenerate mode (same group velocity). Because of reflections at the coupling regions due to surface roughness, both modes couple each other, which break the degeneracy of the modes and a split in resonance occurs. 


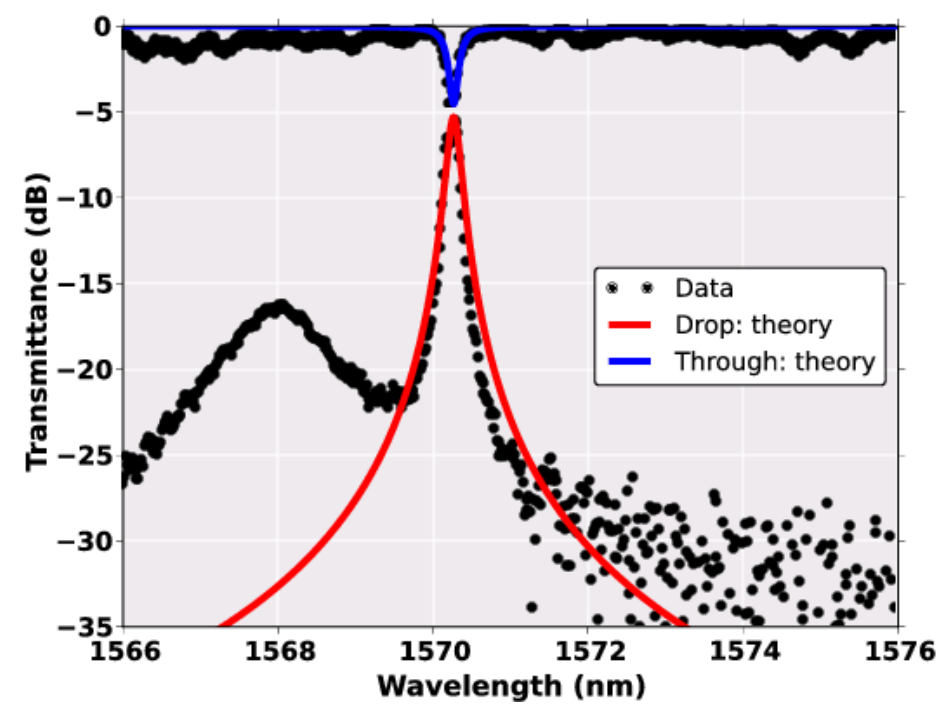

Figure 5.10: Curve fit to data from quasi-TE spectrum measurement of a single micro-ring resonator of $5 \mu \mathrm{m}$ of radius and waveguide to ring gap distance of $200 \mathrm{~nm}$.

The second factor is possibly, the propagation loss induced by the surface roughness of the silicon waveguide structure. This could be expected because after e-beam lithography fabrication no further processing, such as oxidation smoothing, was applied [103], which could improve the smoothness of the waveguide sidewalls.

In figure 5.10 we have a comparison between the theoretical transmittance responses (based on Eq. 2.48 and 2.50 with extracted parameters from Table 5.4) and experimental data. Noise fluctuations in data and ripples came from Fabry-Perot effects due to coupling fiber to the chip optical ports. Theoretical curves are close to experimental values, meaning that the parameters extracted are good approximations of the characteristics of the ring resonator device chosen. However, due to our measurement technique, which had a coarse wavelength resolution in addition to the fabrication quality of the device, we obtained a relative error of the external loss and intrinsic Q factor within $\pm 35 \%$, which is not very accurate. 


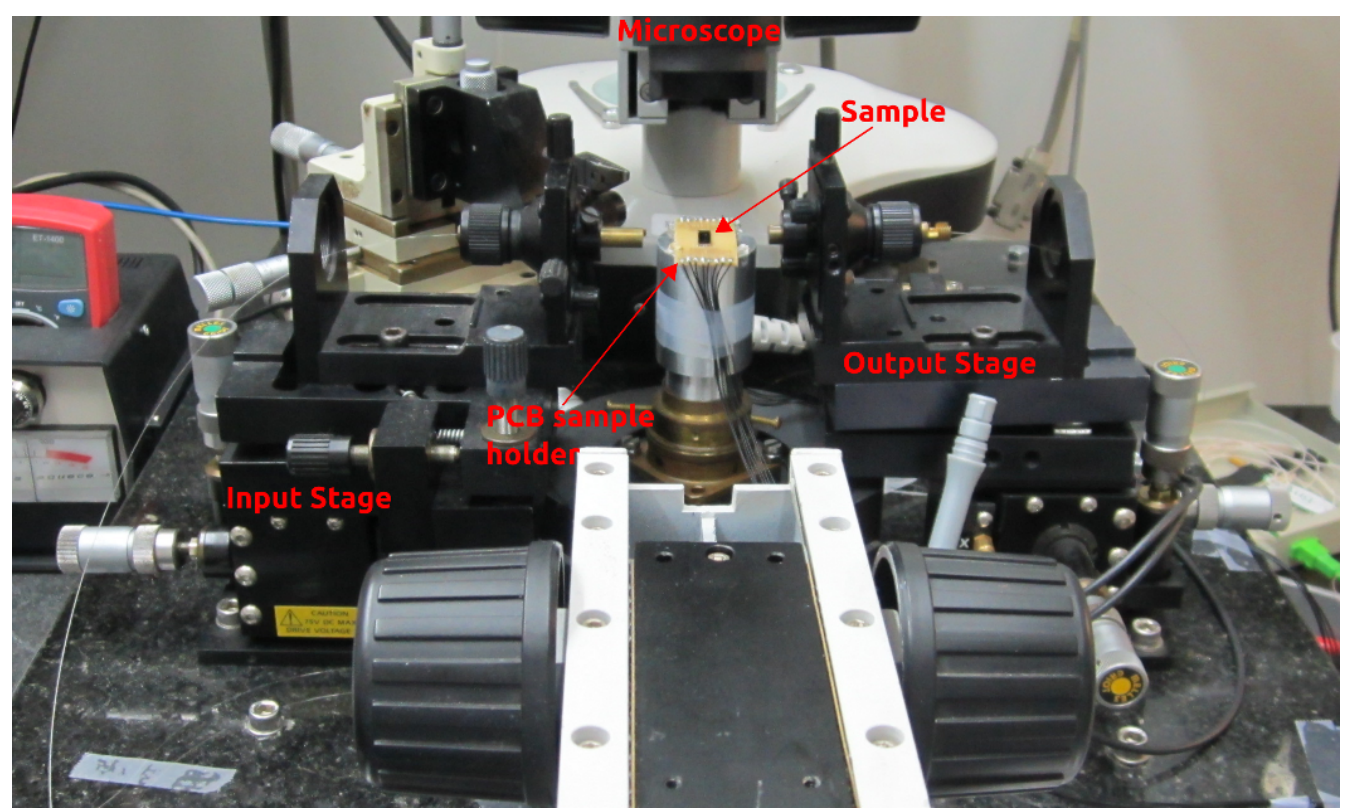

Figure 5.11: Test setup for tuning calibration.

\subsection{Micro-ring resonator wavelength tuning by micro heater}

A micro-ring resonator filter can change its resonance by tuning its passband response. To achieve this, our device was designed to be thermally tuned by controlling the refractive index of the waveguide material. We employed a silicon based waveguide technology because silicon has a large thermo-optic coefficient of $10^{-4}{ }^{\circ} \mathrm{K}^{-1}$.

To test the tuning capabilities of the micro-ring resonator, we applied DC current to a micro heater using a custom built PCB jig as shown in figure 5.11. The PCB jig allowed us to selectively heat single micro-ring devices in a chip by allowing us to inject current into the micro heaters without external probes.

The basic testing setup is illustrated in Figure 5.12. We used a laser diode driver instrument ILX LDC3724 to apply a controlled amount of DC current and prevent any damage on the micro heaters of chip ML2. As it was mentioned in the fabrication section of this chapter, chip ML2 was the one that some "fixes" were done using a FIB to close gaps in the heater films. The amount of applied current started from 0 to $5 \mathrm{~mA}$ in steps 


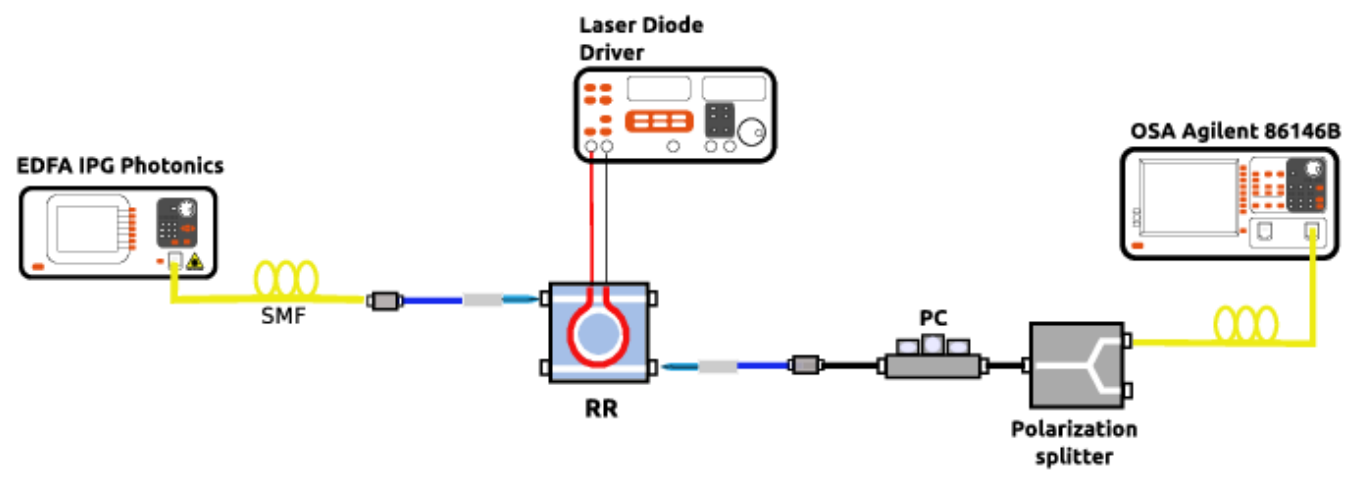

Figure 5.12: Schematic diagram of the thermal tuning characterization of a micro-ring resonator filter.

of $0.5 \mathrm{~mA}$. We recorded the spectrum at the drop port of the ring device after waiting a couple of minutes in order to reach thermal stability.

One important issue to mention is that temperature of the chip was not controlled, which is a factor to consider for measurement repeatability and stability, however the room temperature was maintained by an $\mathrm{A} / \mathrm{C}$ system. Due to time constraints we did not include a Peltier thermo-electrical element below the chip with a thermistor sensor. To be able to do that, we had to make modifications in the PCB and chip wire-bonding to include electrical connections of the Peltier and thermal sensors.

Figure 5.13 shows an overlay graph of the spectrum of the drop port of a ring element (5 $\mu \mathrm{m}$ radius) at different applied DC currents. By increasing the current the resonance is red-shifted, the amount of spectral shift is greater at high currents because actually it is the applied Joule power that controls the temperature of the device element. Because power is proportional to the square of the current, we see a quadratic relation between the wavelength resonance and DC current.

In order to prove our previous comments, Figure 5.14 shows experimental results of the relation between the resonance shift of an micro-ring resonator (MRR) and current squared. This linear regression is accurate with a relative error of less that $1.0 \%$. 


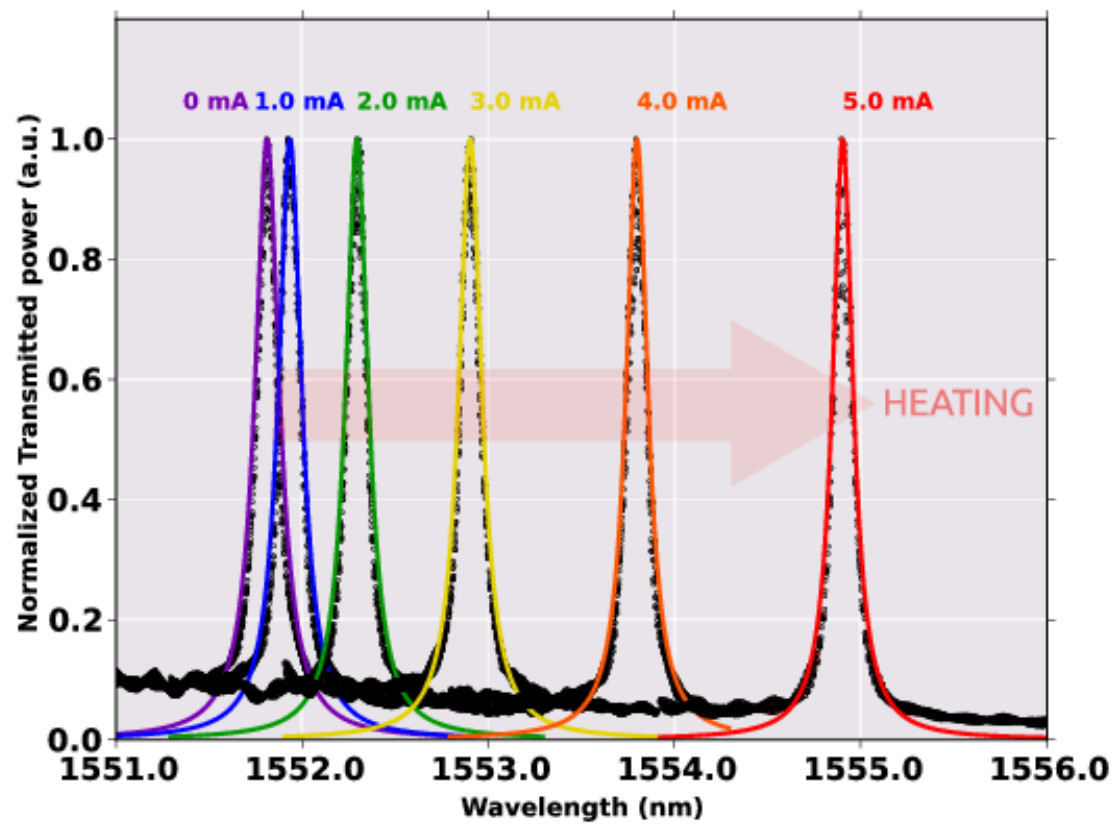

Figure 5.13: Drop port transmission spectrum response after applying DC current (from $0 \mathrm{~mA}$ to $5 \mathrm{~mA}$ ) to a micro heater on top of the micro-ring resonator device. Color lines correspond to Lorentzian curve fit applied to data.

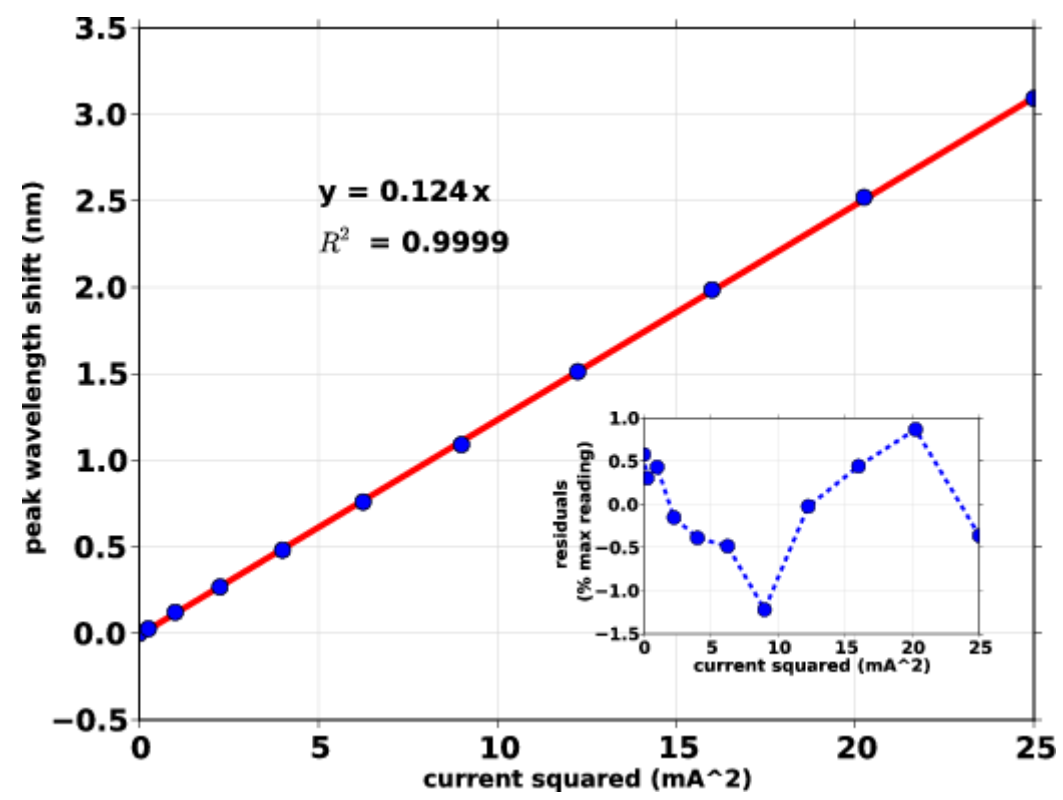

Figure 5.14: A micro-ring resonator resonance tuning response as a function of the square of DC current. 
Resonance tuning therefore was proportional to the square of the current. If we consider the micro heater electrical resistance constant (assuming the resistance change due to temperature as negligible), then the resonance shift is also proportional to the applied power at the micro heater. The slope of the curve in Figure 5.14 was determined to be $0.124 \mathrm{~nm} / \mathrm{mA}^{2}$, which compared to a previous result [3], it had an increase factor of two. We believed this increase in efficiency was obtained because we employed a polynomial shaped micro heater instead of the meander-like shape fabricated and characterized in [3].

In this chapter, some details about the fabrication of a ring resonator filter device were explained, additionally, the fabrication of micro heaters was also described. Some of the fabricated chips showed defects in the micro heaters that were later on fixed using a FIB process to implant Platinum and bridge some gaps. A $5 \mu \mathrm{m}$-radius ring resonator was selected from a collection of devices inside an optical chip. The selected device had a Q factor between 8000 - 10000, and a large FSR of $18 \mathrm{~nm}$ with a tunable range of $3 \mathrm{~nm}$ at 5 $\mathrm{mA}$ heating current. This device after measurement analysis based on models described in chapter 2 , showed a noticeable amount of internal loss due to the fabrication quality. This loss was also a reason on why the external Q factor was a bit low compared to other designed devices found in the literature. Nevertheless, this device showed its potential to be applied as an active element to interrogate optical sensors as it is explained in the following chapter. 


\section{CHAPTER 6}

\section{WAVELENGTH TO TIME INTERVAL DEMODULATION EMPLOYING A TUNABLE SILICON MICRO-RING RESONATOR}

In applications, such as spectroscopy, optical sensing and laser monitoring, precise detection of wavelength changes in any optical spectrum is important in order to quantify a physical measurement or performance characteristic. Wavelength shift detection has received much research attention [16, 104, 105], particularly in fiber Bragg grating (FBG) sensor applications where the physical measurand is wavelength encoded [16]. Common methods for sensor wavelength demodulation are power ratio-metric measurements, Fabry-Perot filtering methods, grating spectrometry and interferometric methods, where the latter have the advantage of offering high precision in terms of wavelength resolution $(\sim 15 \mathrm{pm})[16]$

However, most of those alternatives are usually implemented with bulk optical elements or use long optical fibers. Additionally, they need precise mechanical alignment with good environmental isolation $[43,53]$.

It is expected that any method employing miniaturized optical processing elements has the advantage to offer reduced size and high scalability. Integrated Silicon-on-Insulator (SOI) spectrometers based on Arrayed Waveguide Grating technology were demonstrated previously and used to interrogate Tilted Fiber Bragg grating (TFBG) sensors with a channel spacing resolution of $0.18 \mathrm{~nm}$ [106]. Another interesting candidate for optical processing is the micro-ring resonator (MRR). This device was demonstrated to work as an optical filter, modulator or optical switch $[23,86]$. An advantage of using the micro-ring resonator is that a micro-spectrometer can also be implemented with the capability of obtaining high resolutions by the proper design of its Q-factor.

In this study, the basic theory of a MRR device was explained in Chapter 2, and the design and implementation details were described in Chapters 4 and 5. This chapter presents 
details of a proposed sensor demodulation system that uses a MRR as a tunable filter in order to convert a sensor wavelength shift into a time interval measurement. To control a MRR resonance, thermo-optical tuning is applied. With this type of tuning, a reasonable wide spectrum range is covered at low power. This chapter begins with an introduction of the system concept, followed by an explanation of the time interval demodulation method. A simplified tuning model is presented, which forms the basics to build a simulation algorithm. In order to predict the minimum detectable wavelength for this system, a Monte Carlo simulation was executed. Finally, experimental tests are described and results are analyzed, which served to assess the performance of this wavelength demodulation system when it was applied to a FBG strain sensing scenario.

\subsection{System Concept}

A conceptual diagram of the wavelength demodulation system is shown in Figure 6.1. The key element is a silicon micro-ring resonator (MRR) buried in silicon dioxide ( $\mathrm{SiO} 2)$. In particular, the purpose of this filter device is to scan and monitor the wavelength shift of a sensor, e.g. fiber Bragg grating (FBG), whether a change in temperature or a quasi-static axial strain is applied to it.

The ring resonator has two ports: drop and through. Each port transmittances (transfer functions) shows multiple peaks or valleys. Those are micro-ring resonances and they are separated from each other by a free spectral range (FSR). That value depends on the structural parameters, as explained in Chapters 2 and 4.

In this work, the tuning method chosen for an optical integrated device, such as a MRR, is based on the thermo-optical effect. Thermally tuning a micro-ring resonator produces a shift of its resonance; thus obtaining a scanning operation. This is accomplished by applying current to a micro-heater located on top of the MRR device. The micro-heater is heated and the temperature surrounding the Si micro-ring waveguide in- 


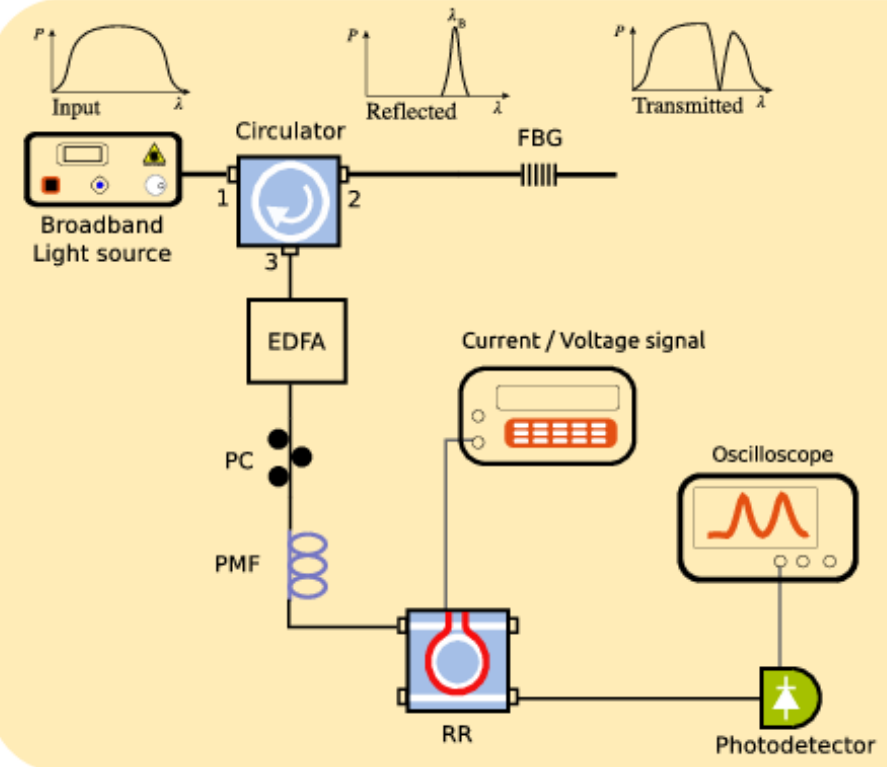

Figure 6.1: Concept diagram of the wavelength detection system based on MRR device. A current source is applied to a micro-heater film on top of a Si MRR with a SiO2 cladding. A FBG sensor is employed to demonstrate the system capabilities to detect Bragg wavelength shifts due to strain or temperature changes in the sensor. Adapted from [72].

creases. Any differential change in temperature induces a large proportional change in the effective refractive index of the silicon waveguide due to silicon's large thermo-optic coefficient. Because the optical phase of the MRR resonance depends on the effective index of the waveguide, a micro-ring resonance wavelength change is directly proportional to temperature $[3,8]$.

To simplify the analysis in the next section, the optical sensor spectrum was assumed to be within one FSR and close to the drop port transmittance. That assumption implies both spectra will overlap each other during a tuning operation. Any overlapped intensity then is captured by a photo-detector, which measures any received optical power as the ring resonance is periodically tuned by a micro-heater. 


\subsubsection{Time Interval Wavelength Demodulation}

Time interval wavelength demodulation was previously proposed for monitoring wavelengths of the spectral peaks of lasers and FBG sensors [21]. In that study, authors employed a mechanically tuned FBG fiber filter acting as a spectrum analyzer to measure optical power as a time domain signal. They demonstrated $1 \mathrm{pm}$ resolution, however, that was achieved at expense of a narrow wavelength scanning range. Another limitation was due to the mechanical nature of the piezoelectric actuator used for filter tuning. Piezoelectric actuation exhibits non-linear effects like hysteresis and creeping, causing slow deformations due to applied loads.

In this section, a system model of the thermal tuning of a micro-ring resonator is presented. Then, an expression is derived for the center wavelength of a sensor using the time interval wavelength demodulation [72].

As mentioned in the previous section, there is a linear relation between a micro-ring resonance and the device temperature. When current is applied to a micro-heater, its temperature is directly proportional to the heating power; thus, there is a direct relation between Joule power and a micro-ring resonance. That relation is found in the literature and expressed as a wavelength sensitivity coefficient $(\alpha)$, which is around $0.068 \mathrm{~nm} / \mathrm{mW}$ for a meander shape micro-heater design [3]. However, the design can be optimized in terms of more $\mathrm{nm} / \mathrm{mW}$ if a circular micro-heater shape is used. By taking into account this linear relationship, the resonance wavelength is [72]:

$$
\lambda_{\text {res }}=\lambda_{0}+\alpha P
$$

where $\lambda_{0}$ corresponds to the ring resonator center wavelength at room temperature, and $\alpha P$ corresponds to the resonance change due to joule heating. 


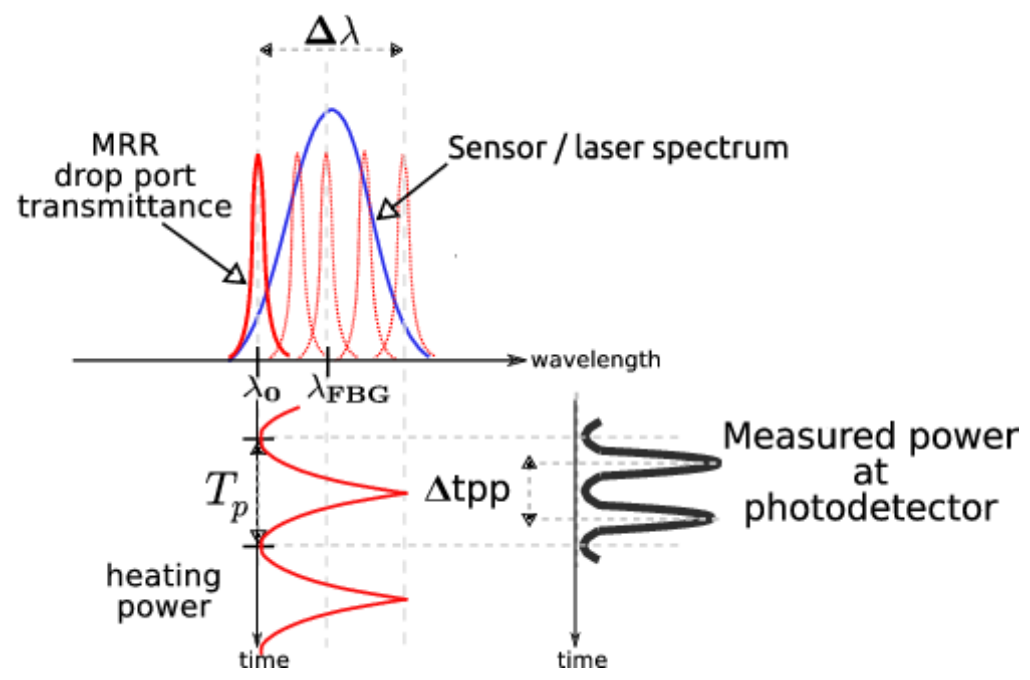

Figure 6.2: Concept diagram of the time interval demodulation scheme based on MRR device (C)2011 IEEE.

A conceptual diagram of the time interval demodulation scheme is shown in Figure 6.2. In simple terms, a ring resonator filter samples a sensor's optical spectrum. The micro-ring filter is tuned around a certain wavelength range $(\Delta \lambda)$ by heating this device periodically with a triangular waveform. The MRR drop port transmittance can be approximated as a Lorentzian function, as demonstrated in Chapter 2. If the spectral region of interest is within one free-spectral-range (FSR), and applying Eq. 6.1 with a three parameter Lorentzian function, we have an expression for a tunable filter spectrum as [72]:

$$
T_{d}(\lambda, t)=\frac{I \gamma^{2}}{\gamma^{2}+\left[\lambda-\lambda_{\text {res }}(t)\right]^{2}}
$$

where $I$ is the maximum intensity, $\gamma$ is the full width at half maximum (FWHM) of the micro-ring transmittance and $\lambda_{\text {res }}(t)$ corresponds to Eq. 6.1.

The overlap between the FBG sensor reflectance and the MRR drop port transmittance produces a power signal, which is measured by a photo-detector, as is shown in Figure 6.2. A current source is applied to a micro-heater film on top of a Si MRR with a SiO2 
cladding. This current flowing through the micro-heater produces heat that will change the temperature of the MRR device tuning its resonance according to heating power. The photo-detector output is captured by an oscilloscope where peaks appear whenever the MRR and FBG wavelength centers match. Therefore, the time interval $\left(\Delta t_{p p}\right)$ between two peaks within a period is a metric related to the center wavelength of the optical sensor spectrum.

An expression that relates a sensor center wavelength and a measured time interval between peaks can be obtained as follows. First, let's consider a triangular voltage signal (with period $T_{p}$ ) applied to a micro-heater. Since micro-heaters are typically made of Nichrome with a temperature coefficient of resistance of $0.4 \times 10^{-3}{ }^{\circ} \mathrm{C}^{-1}$; then, Joule power changes as resistance increases due to temperature. In our model, this issue was not taken into consideration for simplification purposes; thus, resistance was assumed constant. Second, taking into account the linear relationship between resonance and Joule power by applying Eq. 6.1, the micro-ring tuning resonance is:

$$
\lambda_{\text {res }}(t)=\lambda_{0}+\alpha \frac{v^{2}(t)}{R}
$$

By noticing that a triangular signal is used, the expression for resonance change in a MRR within one period becomes [72]:

$$
\lambda_{\text {res }}(t)=\lambda_{0}+\alpha \begin{cases}\frac{4 A^{2}}{R T_{p}^{2}} t^{2} & ; 0 \leq t \leq T_{p} / 2 \\ \frac{1}{R}\left[A-\frac{2 A}{T_{p}}\left(t-\frac{T_{p}}{2}\right)\right]^{2} & ; T_{p} / 2 \leq t<T_{p}\end{cases}
$$

Where $A$ is the maximum voltage amplitude and $R$ is the micro-heater resistance.

Third, looking at Figure 6.2, the power detected waveform has symmetry around half period $\left(T_{p} / 2\right)$; thus, the time interval between peaks is $\Delta t_{p p}=T_{p}-2 t_{0}$, where $t_{0}$ is the time instant at the peak. Any peak is observed when the MRR resonance is equal to the 
optical sensor or laser center wavelength. Applying Eq. 6.4, together with the previous observation, the center wavelength of a target sensor is related to a time interval by [72]:

$$
\lambda_{\text {target }}=\lambda_{0}+\alpha \frac{A^{2}}{R}\left(1-\frac{\Delta t_{p p}}{T_{p}}\right)^{2}
$$

The last equation is a function of MRR resonance at room temperature $\lambda_{0}$, the maximum tuning voltage, the micro-heater resistance and the period $T_{p}$. In summary, this expression predicts the center wavelength of a sensor, such as a FBG, but it can also measure the peak wavelength of any other bandwidth limited signal, including that of a laser line.

\subsection{System Simulation}

A numerical simulation was developed in order to test some characteristics of this wavelength demodulation system $[20,72]$. The main simulation was divided in three parts: spectrum modeling, spectrum overlap integration and peak to peak search with time interval calculation. All of them were implemented in Python programming language with scientific and numerical analysis modules [92, 93, 94].

Figure 6.3 shows a block diagram of the steps done in the system simulation. The controlling signal is a voltage applied to a micro-heater, where the Joule power is proportional to the square of the voltage. The basic tuning model of the micro-ring drop port explained in the previous section was applied. That model provides the mechanism for tuning the resonance at the drop port of a micro-ring resonator. This simulation executed calculations in the spectral domain; thus a wavelength range of $18 \mathrm{~nm}$ was set. The tuning voltage signal was sampled at a rate of $200 \mathrm{Ksamples/s.} \mathrm{The} \mathrm{maximum} \mathrm{wavelength} \mathrm{reso-}$ lution of this simulation was $0.22 \mathrm{pm}$; this was set according to the wavelength range and the frequency of the signal. Finally, the spectral modeling was completed by defining a 


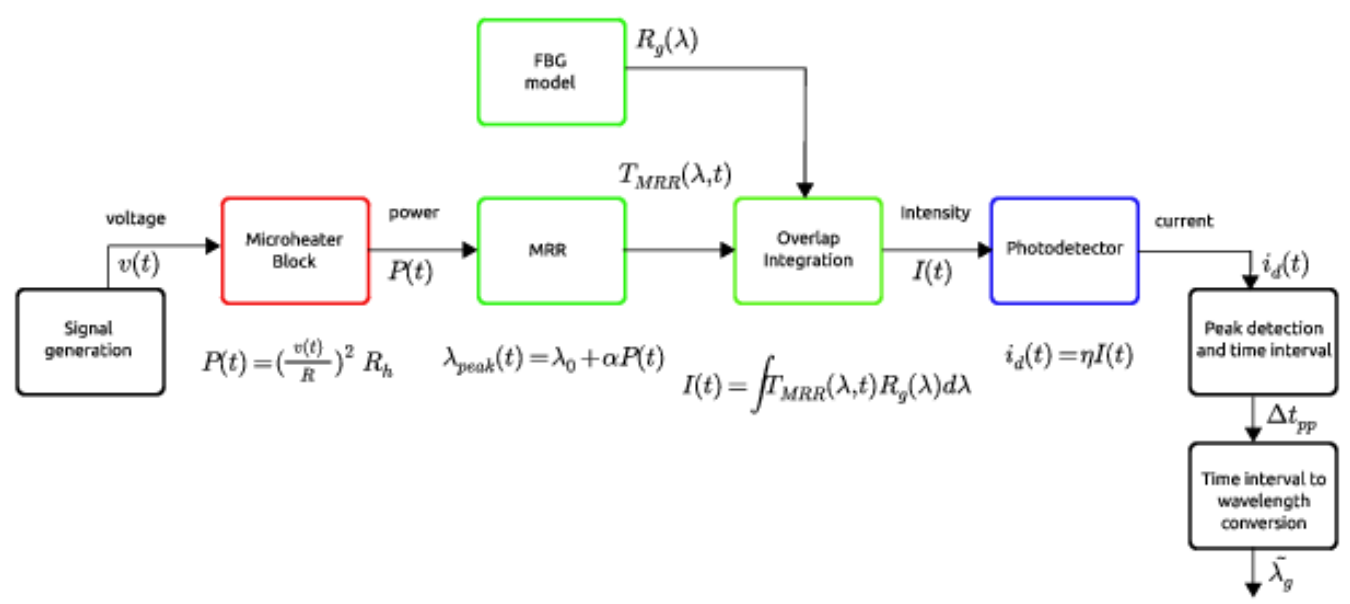

Figure 6.3: Simulation algorithm block diagram.

model for the FBG spectrum as a Gaussian function ${ }^{1}$ with a bandwidth of $0.2 \mathrm{~nm}$, unitary intensity and with a center wavelength within the tuning range of the MRR.

The overlap between the drop port transmittance and a FBG spectrum was obtained by numerical integration. This value was calculated at each time sampling step to get a signal that represents detected intensity. The last simulation step involved a maximum value search algorithm (peak-search) applied to the simulated intensity signal. Finally, another software routine identified the time at consecutive peaks and recorded their difference as a time interval.

\begin{tabular}{|c|c|c|}
\hline Name & Symbol & Value \\
\hline \hline Resonance wavelength $\left(\right.$ at $\left.22^{\circ} \mathrm{C}\right)(\mathrm{nm})$ & $\lambda_{0}$ & 1573.98 \\
\hline Max. temperature change $\left({ }^{\circ} \mathrm{C}\right)$ & $\Delta T_{\max }$ & 26.9 \\
\hline Linear resonant shift coefficient $\left(\mathrm{nm} /{ }^{\circ} \mathrm{C}\right)$ & $a$ & 0.095 \\
\hline Ring FSR $(\mathrm{nm})$ & $F S R$ & 18 \\
\hline Ring FWHM (nm) & $B_{R}$ & 0.15 \\
\hline Heating current freq. $(\mathrm{Hz})$ & $f$ & 1.0 \\
\hline FBG FWHM (nm) & $B_{G}$ & 0.33 \\
\hline FBG wavelength separation $(\mathrm{nm})$ & $\Delta$ & 0.2 \\
\hline
\end{tabular}

Table 6.1: Numerical simulation parameters of a MRR sensor interrogator.

\footnotetext{
${ }^{1}$ FBG reflectance as a Gaussian function model was explained in chapter 3
} 
The main simulation parameters are summarized in Table 6.1. Some values were extracted from experimental results, as reported in [3], while the maximum tuning voltage and tuning frequency were chosen arbitrarily.

\section{Wavelength scan simulation and demodulation using time interval measurements}

Results from simulations are shown in Figure 6.4. The blue and red colored curves represent various overlap spectra from two different center wavelengths of spectrally encoded sensors. Also, the dotted line corresponds to the MRR wavelength shifts while the device is tuned. As expected, this curve follows the instantaneous joule power when a triangular voltage is applied to the micro-heater.

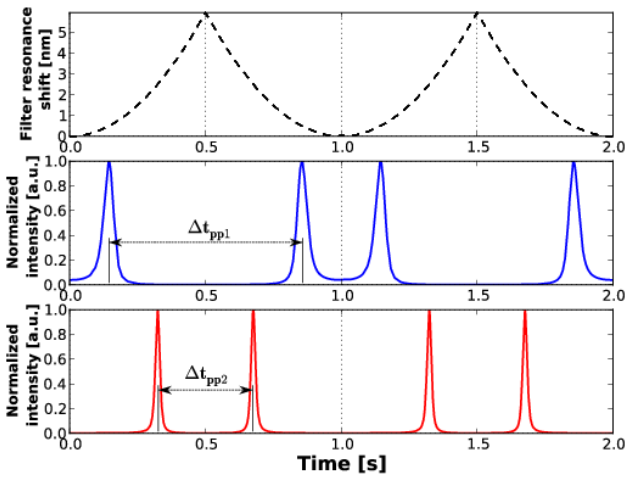

(a)

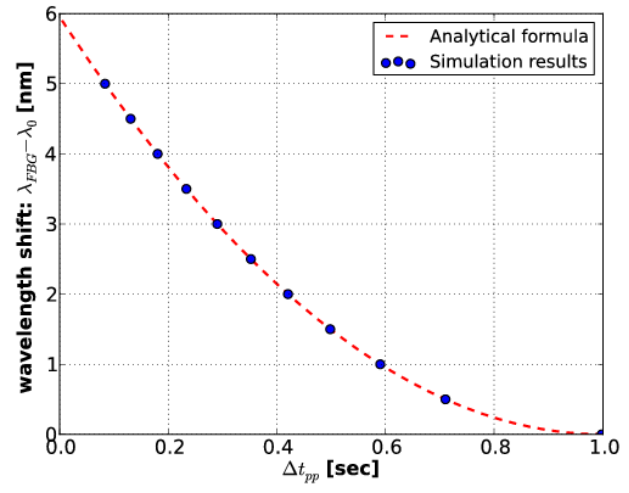

(b)

Figure 6.4: (a) Simulation results. The upper plot shows the MRR filter resonance tuning signal, the middle and lower plot shows the detected intensity signal after spectrum overlap of MRR filter transmittance and FBG reflectance at two wavelength shifts $(0.5$ and $2.5 \mathrm{~nm}$ ). (b) Results of time interval between peaks at 11 wavelength shifts after system simulation. The line corresponds to Eq. 6.5 (c)2011 IEEE.

From the overlap graphs in Figure 6.4(a), e.g. by looking at the blue curve, for any FBG sensor close to the initial resonance of the MRR filter, its intensity signal becomes wider and a bit distorted. As the FBG center wavelength goes far away from initial MRR resonance, this bandwidth becomes narrow. This observation can be explained by looking 
at the MRR tuning wavelength from the dotted line curve. Wavelength tuning does not follow a triangular shape as the tuning voltage; thus, a MRR resonance change at half period is not linearly increasing/decreasing, but rather it has a quadratic behavior. The reason is due to thermo-optic tuning, which depends on electrical power rather than voltage (or current). That behavior widens the spectrum at regions in time close to zero and one period when the wavelength tuning is slow. On the other hand, at regions close to half period, tuning is faster due to increased voltage at the micro-heaters.

The spectrum distortion at some regions in time does not fundamentally affect the demodulation operation, which is based on measuring the peak-to-peak time interval as shown in Figure 6.4(a) represented as $\Delta t_{p p 1}$ and $\Delta t_{p p 2}$.

Figure 6.4(b) illustrates the relationship between the peak to peak time interval and sensor wavelength shift. Dots correspond to results taken from numerical simulation at 11 different FBG positions (by emulating shifts in Bragg wavelengths). The red line corresponds to predicted wavelength values after applying Eq.6.5.

\section{System noise performance}

The system performance when noise is present was simulated using a Monte Carlo method. The purpose of applying this method was to be able to investigate the minimum wavelength shift that this interrogator can detect when the signal is corrupted by Normal distributed random noise. At the detector, there are two kinds of noise: thermal and shot noise. Shot noise usually is characterized as a Poisson process while thermal noise is Gaussian. Due to its simplicity, we choose Gaussian distributed noise and apply it to a Monte Carlo technique to find statistical variations of the peak of simulated signals.

To accomplish that, we set up another numerical experiment using our previous simulation implementation with the same parameters as Table 6.1. At a fixed FBG center 
wavelength, we considered two simulation variables: one was the Q factor with values from $1 \times 10^{3}$ to $5 \times 10^{4}$, and another was the signal-to-noise ratio (SNR), with selected values between 10 and $40 \mathrm{~dB}$. For each SNR value, we generated a normal distributed random noise signal added to the computed intensity signal. A time interval between peaks was extracted from the noisy signal to obtain an estimated wavelength value. The process was repeated 100000 times per each parameter in order to obtain a statistic of the difference in the simulated wavelength value between noiseless and noisy conditions. That repetition number selected depends on the precision of the averaging, which in this case is within $0.3 \%$. The minimum wavelength shift error can be obtained by computing the standard deviation or root mean squared of the wavelength difference between peak wavelength detected with noise and Bragg wavelength without noise. In other words:

$$
\delta \lambda_{\text {min }}=\sigma=\sqrt{\frac{\sum_{i=1}^{K}\left(\lambda_{i}-\lambda_{B}\right)^{2}}{K}}
$$

Results from Monte Carlo trials are shown in Figure 6.5(a). A logarithmic downward trend of the wavelength precision can be observed as system SNR increases. Performance is very poor when using wide bandwidth filters (low Q factor). It is therefore expected that much better precision can be obtained with high Q filters at high SNR. However, performance cannot be further improved if the bandwidth of the sensor is not also considered. In Figure 6.5(b), results of the wavelength difference at different FBG bandwidths are shown together with some values of MRR Q factors. The statistical uncertainty is reduced even at sub-picometer levels when narrow-band sensors and high Q factor filters are used. 


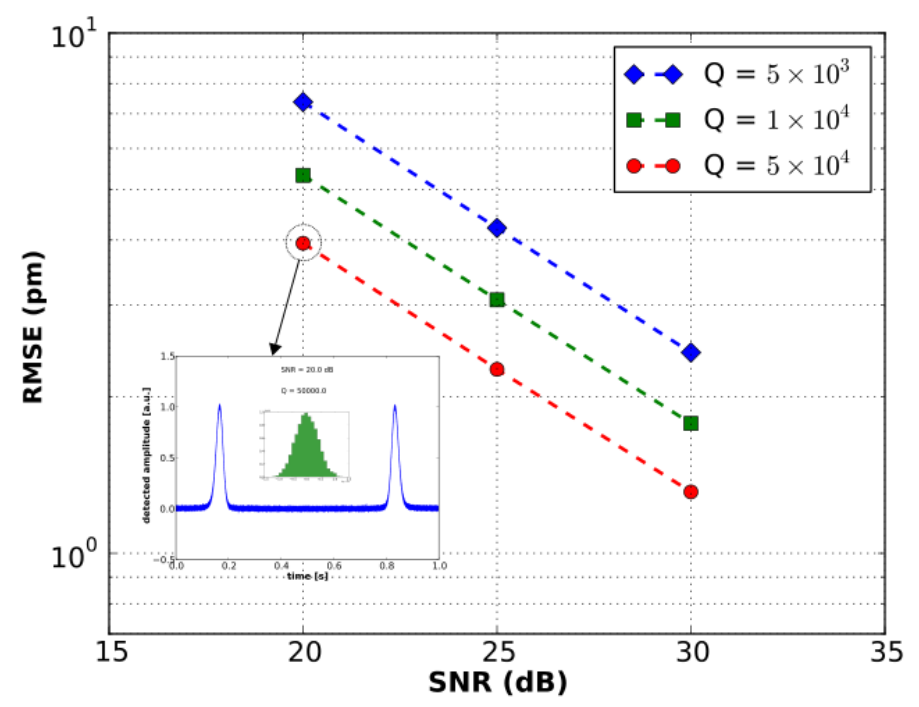

(a)

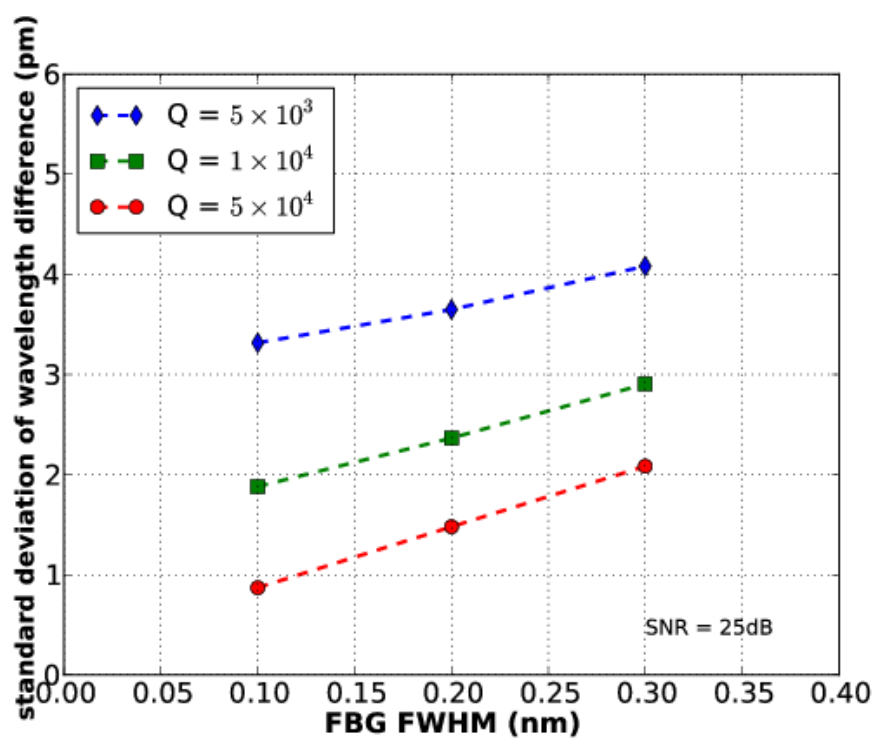

(b)

Figure 6.5: (a) Results of standard deviation (root mean squared error - RMSE) of detected wavelength as a function of SNR at different MRR total Q values. FBG Bragg wavelength was simulated with a wavelength shift of $1.0 \mathrm{~nm}$ from a simulated MRR resonance of $1539.04 \mathrm{~nm}$. (b) Results of root mean squared error of detected wavelength as a function of an optical sensor full-width-at-half maximum (FWHM) (c)2011 IEEE. 


\subsection{Sensor interrogation experiments.}

This section presents the application of a micro-ring resonator filter as an interrogator through a series of experimental tests. Details on the fabrication and characterization of this device were explained in the previous chapter. Here, the drop port resonance of a micro-ring resonator was tuned around a certain wavelength range $(\Delta \lambda)$ by heating this device periodically with an electrical current signal.

\section{Calibration measurement using a tunable laser.}

An experimental interrogation system was set up first in order to detect the wavelength of a laser source; thus obtaining a relation between the wavelength and time interval measurement useful as calibration. The system setup is shown in Figure 6.6. A narrowband (1.3 - $1.6 \mathrm{pm}$ bandwidth) tunable laser (SANTEC ECL-210) was selected as optical source. Light from the laser was coupled into the input port of a micro-ring resonator (MRR) device. That device was previously characterized (see chapter 5) and two types of resonances were present: a quasi-TE and quasi-TM modes. It was possible with a polarization controller to select the polarization mode of interest. In this experiment, a quasi-TE mode was manually selected with a resonance of $1551.8 \mathrm{~nm}$ (at room temperature). The bandwidth (FWHM) at that resonance was $0.14 \mathrm{~nm}$.

The chip containing our ring resonator was mounted on a printed circuit board (PCB) with the chip feed-lines wire-bonded to the PCB electrical traces. That construction allowed us to use regular AWG-22 electrical wires and connect our chip to a laser diode driver (ILX LDC 3724B) working as a current source.

The measurements started by setting the laser at a wavelength within the tuning passband of the MRR. Values ranging from $1552 \mathrm{~nm}$ to $1554.2 \mathrm{~nm}$ were chosen as target wavelengths. A triangular signal (from a HP8116A function generator) with a voltage 


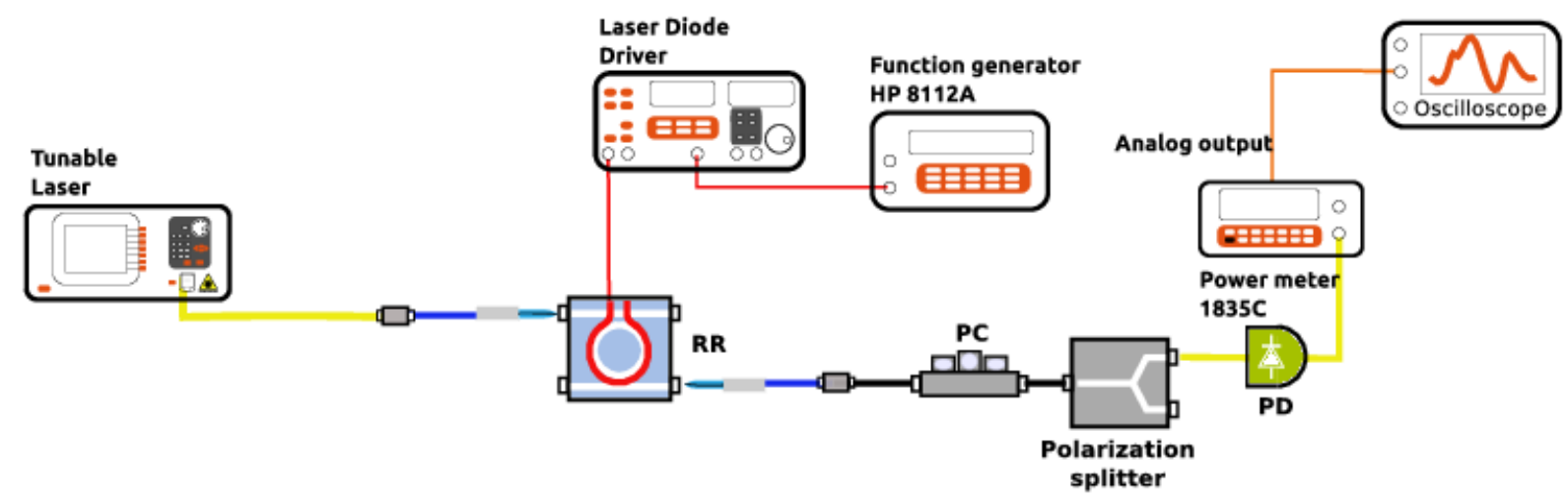

Figure 6.6: Schematic diagram of time interval measurement setup using a laser source for calibration purposes.

from 0 to $255 \mathrm{mV}$ at $0.5 \mathrm{~Hz}$ was applied in order to modulate the laser diode driver. This laser driver converted the input voltage signal into a current signal with a conversion factor of $20 \mathrm{~mA} / \mathrm{V}$, therefore, the maximum peak-peak current was $5 \mathrm{~mA}$. The current signal was fed into a micro-heater, which changed the temperature of the micro-ring resonator; therefore, the micro-ring (MRR) resonance was tuned up to $3 \mathrm{~nm}$. This MRR tuning effect allowed scanning and capturing the laser spectrum. The scanned optical signal was collected from the drop port of the MRR device through a lensed fiber and with a manual polarization controller together with a splitter, it was possible to select the TE polarization state. Finally, the splitter output was connected to a power meter (Newport 1835C) whose analog output was fed into a digital oscilloscope, thus capturing the scanned signal.

Figure 6.7(a) shows oscilloscope curve traces at three specific laser wavelengths. Those scanning traces came from the overlap intensity measured by the photo-detector. The scanned spectrum traces in that figure have multiple peaks due to the periodic tuning of the micro-ring filter (a triangular current signal was used).

Also, there are some small and wide peaks ("bumps") in the scanned spectrum. Those come from the overlap effect between residual TM modes and the laser line. Recalling from chapter 5, results from measurements of a ring resonator device (from chip ML2) showed a TE/TM mode separation of $2 \mathrm{~nm}$. Because the TM mode was a bit closer to the 
TE mode, then the TM mode interferes with the scanning process of the laser spectrum, which gave as a result those "bumps" in the traces. It is worth mentioning that the effect of the TM mode can be reduced by properly adjusting the polarization controller.

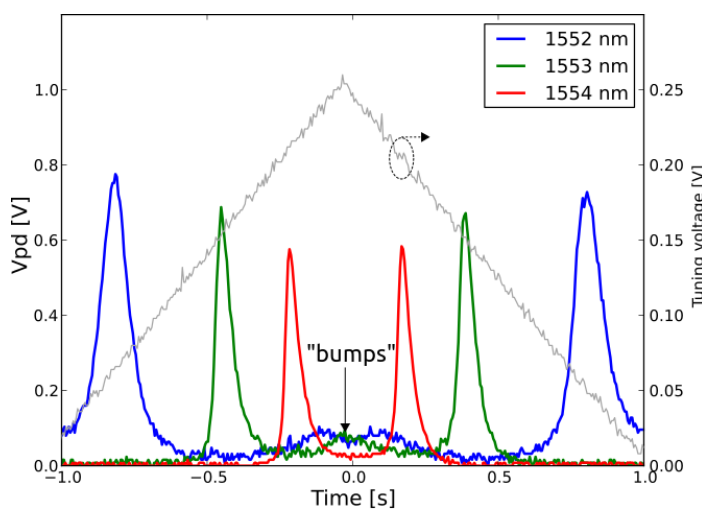

(a)

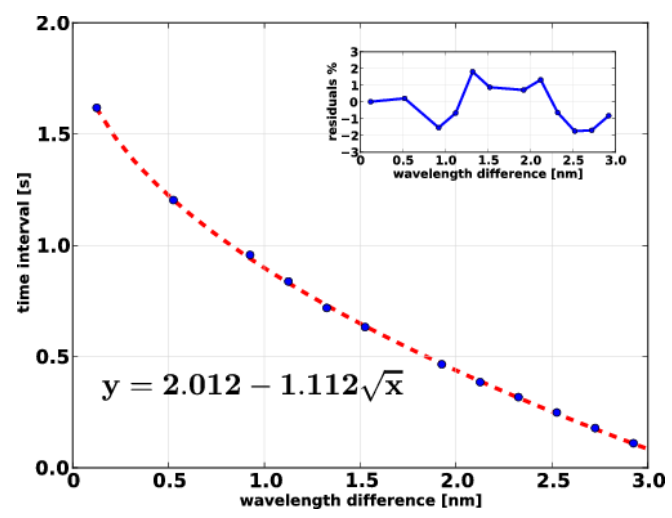

(b)

Figure 6.7: (a) Oscilloscope traces of three laser lines after being scanned by a tunable ring resonator and selecting the TE mode response. The time interval in one period of the tuning (scan) triangular signal (shown in gray color) was measured by detecting the peaks of those traces. The "bumps" in between two peaks (near the center) represent the effect of residual TM mode, which can be reduced by adjusting a polarization controller. (b) Time interval measurement calibration curve for known laser wavelength values.

Figure 6.7(b) shows a plot of the experimental peak to peak time interval measurements corresponding to the difference between the laser wavelength and initial resonance of the ring (e.g. $x=\lambda_{\text {laser }}-\lambda_{M R R}$ ). Also, a red line is shown, which corresponds to a curve fit to measured data. That curve closely follows the trend of the experimental data. The reason of that low error estimation is the narrowness of the laser and the clear peaks in the oscilloscope traces. A source of uncertainty is any double-peak shape ${ }^{2}$ of the measured spectra. This introduces errors in the time interval measurement, which is based on maximum search algorithm. The chart below the main graph shows the difference between the experimental data and the curve fit. The maximum relative error in the estimation is below $+/-2 \%$.

\footnotetext{
${ }^{2}$ This shape was observed in this device as it was explained in Chapter 5
} 
The model used in this calibration was based on Equation 6.5, if we re-arrange that equation as a function of the variable $x=\lambda_{\text {target }}-\lambda_{0}$ the time interval can be expressed as:

$$
\Delta t_{p p}=T_{p}-\sqrt{\frac{R T_{p}^{2}}{\alpha A^{2}}} \sqrt{x}
$$

From the above equation and compared to Figure 6.7, we can easily observe that the estimation of the period $T_{p}$ is around $2.012 \mathrm{~s}$, which corresponds to the period set in the

function generator, and the factor $K=\sqrt{\frac{R T_{p}^{2}}{\alpha A^{2}}}$ is $1.112 \mathrm{~s} / \sqrt{n m}$. Those parameters from this calibration will be used later on to determine the wavelength difference $x$ of a strained FBG sensor compared to the initial MRR resonance.

\section{FBG sensor strain measurements}

In order to use the time interval method with a micro-ring resonator tunable filter sensor interrogator, it was necessary to have a characterized FBG optical sensor. A collection of FBG's with different Bragg resonances were fabricated at the Institute of Advanced Studies (IEAv) in Brazil. A couple of those sensors with resonances close to the micro-ring resonator resonance (1551.8 at quasi-TE polarization) was chosen. A test setup shown in Figure 6.9 was used to carry out strain calibrations of those sensors. In this procedure, the FBG was fixed at one end of the grating and in the other end weights were suspended by placing them into a plastic recipient. The applied axial strain was proportional to the mass suspended, and it can be calculated using:

$$
\varepsilon=\frac{m g}{A \times Y}
$$




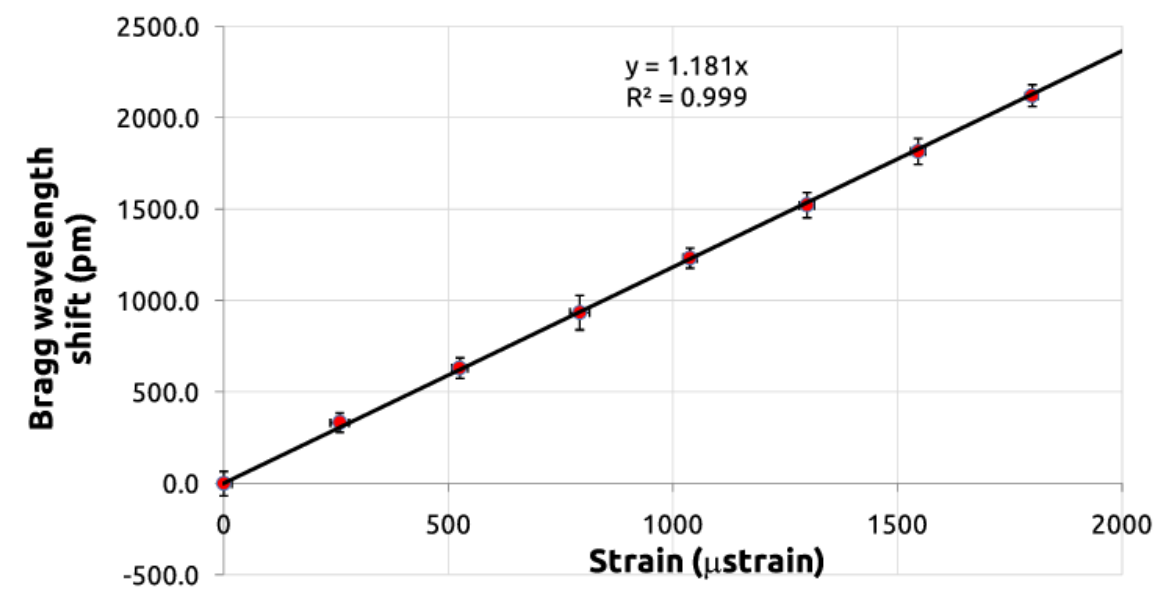

Figure 6.8: FBG strain calibration curve. The approximated strain sensitivity at 1552.821 nm was $0.758 \times 10^{-6} \mu \varepsilon^{-1}$.

where $\mathrm{m}$ is the mass, $\mathrm{g}$ is the gravitational acceleration constant $(9.8 \mathrm{~m} / \mathrm{s})$, A is the cross-section area of the FBG fiber, and Y is the Young's modulus (72.2 GPa for fused silica optical fiber).

Our FBG was pigtailed with a fiber connector, which in turn was connected to an optical circulator. One path of the circulator was fiber connected to a broadband Erbiumdoped Fiber Amplifier light source and the other path was connected to an Optical spectrum analyzer (OSA). Initially, the procedure started with a baseline measurement of the optical reflectance of the FBG when no weight was added (just the weight of the cup and splices). After that, we increased the weight and captured the FBG reflectance with the OSA. The process was repeated in order to get the following weights: $23,48,72,94,118$, 140, 163 and 186 g. OSA measurements of the FBG peak reflectance were acquired only after adding weight and waiting 1 to 2 minutes in order to avoid measurement fluctuations when the weights were put into the recipient.

Figure 6.8 shows the strain calibration curve as a result from the measurements of a FBG with applied weights. In that figure, the $\mathrm{x}$ axis represents the amount of strain, which was calculated based on equation 6.8. The y axis corresponds to the measured wavelength 


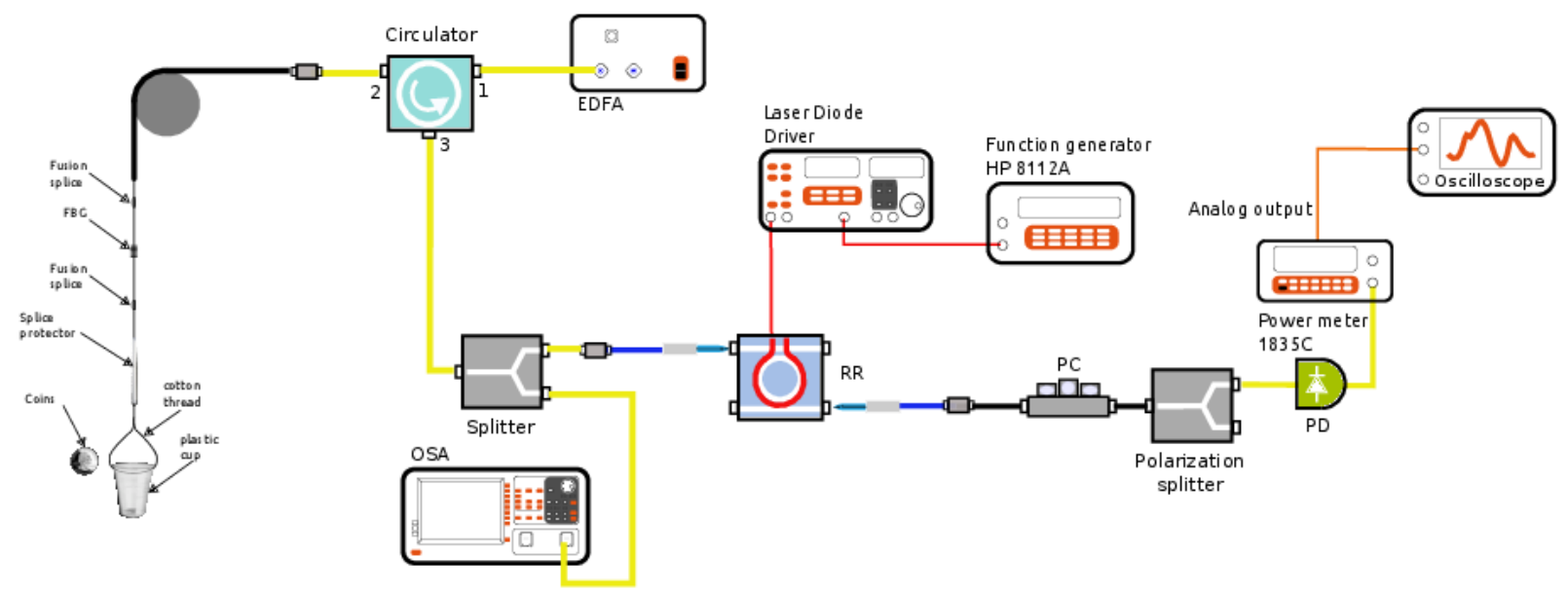

Figure 6.9: Schematic diagram of optical sensor characterization measurement. On left side, a setup is shown where axial strain was applied to a FBG sensor using a plastic cup and coins as weights.

shift of the FBG from the initial (no strain) Bragg resonance of $1552.821 \mathrm{~nm}$.

According to the results of a linear interpolation, we have a slope of $1.181 \mathrm{pm} / \mu \varepsilon$, which divided by the initial Bragg resonance, yields an approximately FBG strain sensitivity of $0.758 \times 10^{-6} \mu \varepsilon^{-1}$, this is in close agreement with other published results in the optical sensor literature $[15,50]$.

\section{FBG sensor interrogation using time interval method}

After FBG sensor calibration, we applied the time interval setup to perform measurements of the same FBG sensor at different weights (strains). Figure 6.9 shows a schematic of the setup, which is similar to the one used for time interval calibration with a laser.

Although the setup was similar to the calibration with a laser, the main difference was the usage of the FBG sensor reflectance as the input to the MRR interrogator. One thing observed was the FBG sensor had a poor reflectivity and its optical spectrum was wide (in terms of 1-2 nm). This situation makes a bit difficult to clearly observe narrow peaks 
in the oscilloscope trace, therefore errors in the wavelength estimation from time interval measurements were expected.

An example set of oscilloscope traces are found in Figure 6.10. Colored lines correspond to the overlap intensity between a strained FBG sensor (at 3 different strains) and the drop port transmittance (in TE mode) as the ring resonator is tuned by a triangular current signal. On those traces, their respective amplitudes go from a low peak voltage to a high peak voltage due to the non-flat amplification region of the EDFA used to interrogate the FBG sensor. At a strain of $259 \mu$ strain, there is a residual TM trace, which was also observed in Figure 6.7(a) when a laser source was applied. Although in the laser calibration, the residual TM trace was smaller than this case, a possible reason for this observation is attributed to a lack of manual optimization of the polarization controller.

As mentioned in the previous paragraph, the spectral shape of the sensor after MRR filter scanning is an important factor, which helps in estimating a sensor wavelength shift. Peak detection data processing algorithms play a significant role in this time interval method. In this chapter, simulations and experimental time interval calibration with a laser simply used a maximum search algorithm to measure the time interval between peaks. Maximum peak data processing relies on searching the time value where a maximum amplitude in the oscilloscope trace occurs. However, this algorithm, while fast and simple, is prone to errors in wavelength estimations. An example of this is depicted in Figure 6.11(a), where a comparison is made between the estimated FBG wavelength shift using time interval between peaks technique and the FBG calibration curve fit as demonstrated in Figure 6.8. Using the maximum peak search algorithm shows outliers in the FBG shift data at strain values of 610 and $1250 \mu$ strain. Those are indicative of how sensitive is this search algorithm to noise and spectral sensor shape asymmetry as was observed in the oscilloscope traces.

To improve peak detection and determine accurately any FBG wavelength shift, an- 


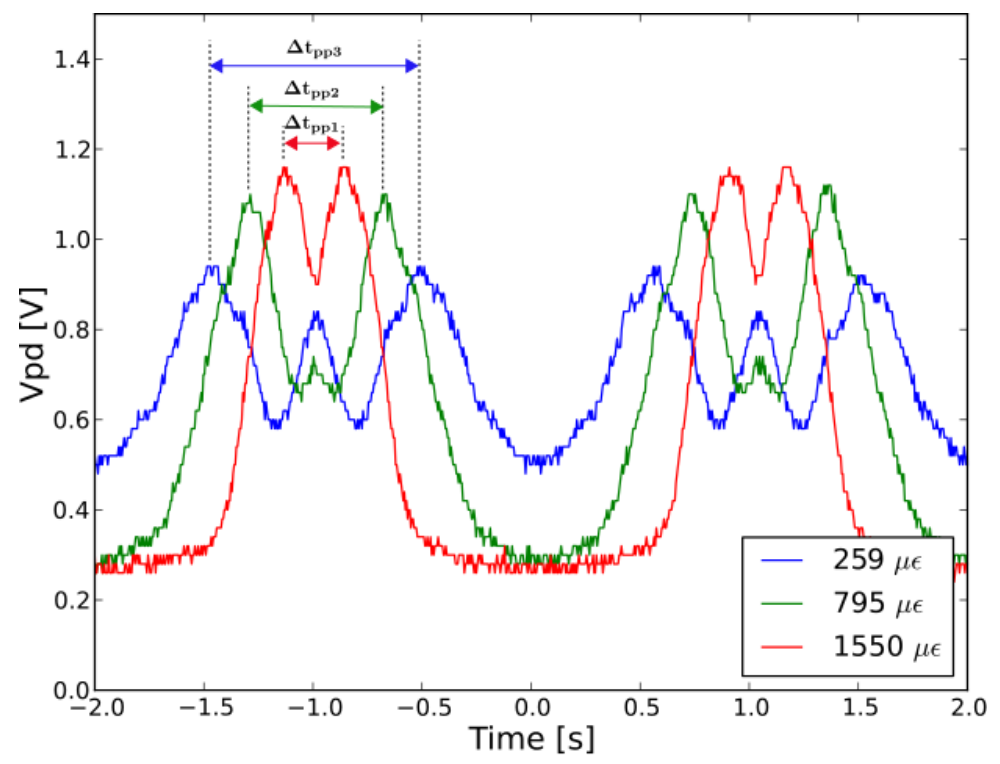

Figure 6.10: Oscilloscope traces showing interrogation of an strained FBG sensor.

other algorithm was applied on the scanned data traces. From Chapter 3, a FBG sensor spectrum can be approximated as a Gaussian function $[66,67]$. Therefore, in this particular experiment, we applied a Gaussian curve fit to each strain related scanned traces. The curve fit was based on a least mean squared algorithm implemented in Python language [94]. The Gaussian model applied allow us to determine the time values where the center peaks occur and from that we obtained the time interval between consecutive peaks within a scanning period.

Using this Gaussian curve fitting gave us an improvement in residual error, as shown in Figure 6.11(a) (red dots). The wavelength difference between the estimated FBG shifts and the strain calibration linear interpolation is shown in Figure 6.11(b). From that graph, the maximum peak search results have a maximum error within $200 \mathrm{pm}$. Compared to the Gaussian fit peak extraction, residuals are much better, producing a root mean squared error within $22 \mathrm{pm}$. 


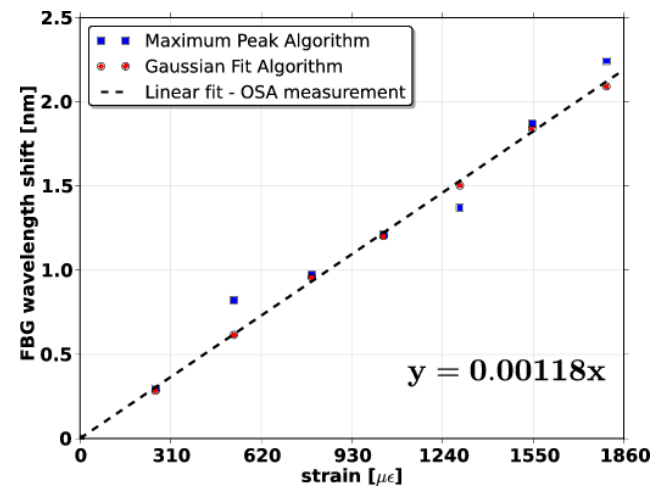

(a)

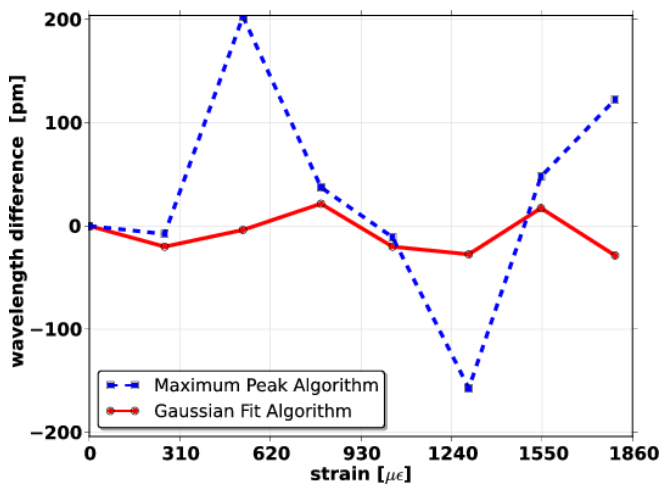

(b)

Figure 6.11: (a) Comparison between time interval results from FBG interrogation and given by linear fit from OSA measurements. (b) The difference error from estimated wavelength shifts using time interval measurements and linear fit from OSA measurements. Blue line corresponds to estimations made with Maximum peak algorithm and Red line comes from Gaussian fit Algorithm.

\section{Discussion and Analysis}

Despite the fact that the FBG sensor was not an adequate one because of its low reflectance $(\sim 10 \%)$ and its wide bandwidth $(1-2 \mathrm{~nm})$, the estimated wavelength shift due to FBG axial strain showed an error of $22 \mathrm{pm}$ compared to Optical Spectrum Analyzer measurements (at $20 \mathrm{pm}$ resolution).

An important observation was that the maximum peak search algorithm used to determine the time interval between peaks metric was prone to deliver large deviations in the estimation of a FBG wavelength shift. The main reason was that the scanned traces from the oscilloscope showed asymmetries and noise fluctuations, which prevented the correct determination of the peaks. The asymmetries of the traces can be attributed to the lack of a clear narrowband spectral peak of the FBG sensor used in the experiment and also to the presence of double peaks in the MRR resonance ${ }^{3}$.

\footnotetext{
${ }^{3}$ The MRR in this experiment showed some spectral characteristics as explained in chapter 5, there were some double-peak resonances due to counter-directional coupling, which came from the roughness at the lateral faces of the waveguides.
} 
An additional cause of the difficulty of detecting peaks from traces with a maximum search algorithm is the apparent noise in the signal, as shown in Figure 6.10. Low SNR in the signal is attributed to the quantization noise of the oscilloscope, additionally, the insertion loss of the optical chip was large $(\sim 20 \mathrm{~dB})$ because optimized alignment was difficult to achieve with the mechanical stages used in the experiment. On the other hand, alignment problems can be properly managed by using nanometer-precision stages. Fiber optic alignment and coupling are common issues in silicon photonic chip devices due to the size of the optical waveguide elements and the refractive index difference of the materials used.

A Gaussian linear fit was applied to the detected oscilloscope traces and the variability/deviations compared to OSA measured data were reduced considerably. This observation was also confirmed in other studies involving FBG measurements $[67,107,108]$. The reason a Gaussian fit yield good results compared to a simple peak search is that FBG sensors have an spectral profile similar to a Gaussian function.

Tuning range of the interrogator depends on the amount of heating applied to the MRR. With just $5 \mathrm{~mA}$, our device was tuned up to $3 \mathrm{~nm}$ in order to track a FBG sensor with a strain up to 2000 microstrain, which is acceptable for practical applications. Other MRR (with 10 um diameter) of similar characteristics as ours had a maximum wavelength tuning up to $6.4 \mathrm{~nm} \mathrm{[3].} \mathrm{Using} \mathrm{Vernier} \mathrm{effect} \mathrm{in} \mathrm{double} \mathrm{ring} \mathrm{structures} \mathrm{it} \mathrm{was} \mathrm{possible} \mathrm{to}$ fine tune their resonance at $3.2 \mathrm{~nm}$ (for ring radii at $10 \mu \mathrm{m}$ and $9 \mu \mathrm{m}$, respectively) [109], in another research by our group we demonstrated Vernier effect with two ring resonators (10 $\mu \mathrm{m}$ and $15 \mu \mathrm{m}$ radii, respectively) that was tuned up to $8 \mathrm{~nm}$ [84], although the polarization state used was TM.

Limitations of tuning range depends on how much current can support the microheaters. Problems like electro-migration and oxidation [10] impose a limit of the maximum current at microheaters. 
The resolution of the interrogator is directly related on the spectral characteristics of the micro-ring resonator. A ring resonator filter takes samples of the spectrum of light that passes through the device. Highly selective micro-ring filters can be obtained by controlling the amount of coupling between the ring and bus waveguides, however the maximum drop efficiency is reduced, thus the amount of collected /extracted light from the input port becomes small. An interesting device that overcomes this issue is the micro-donut resonator [110], which is a silicon disc element with a hole in the center. One feature is that the fundamental mode in a micro-donut travels at the external periphery of the disc and contrary to the micro-ring resonator, it only interacts on the external sidewall close to the bus waveguide. This interaction increases the Q-factor of the device while increasing its drop efficiency significantly [110, 111].

\section{Summary}

In this research, we have demonstrated a small size $(12 \mu m \times 12 \mu m)$ SOI ring resonator optical sensor interrogator based on the time interval between peaks measurement. This device was embedded in a $7.8 \mathrm{~mm} \times 3.8 \mathrm{~mm}$ chip and it has the potential to independently interrogate optical sensors, such as Fiber Bragg gratings, by adding many parallel ring resonator devices thanks to silicon photonics large scale integration technology. Another use of this system is as a method to track resonance of other sensor-on-a-chip devices or laser sources $[69,112]$

A system model was developed, which help us to prepare a simulation and predict some performance characteristics of the system. All those simulations were implemented using open-source numerical tools. The scanning operation was simulated and the performance due to noise also was considered. 
Results indicated that narrow-band filters and sensors help in reducing wavelength error estimations when applying the time interval technique.

Two experiments were demonstrated in this chapter. One represented a calibration procedure by using a tunable narrow-band laser as a light source. With the calibration curve, we obtained a means to calculate an estimation of the wavelength difference value between the MRR resonance and a light source. The second experiment involved the application of a FBG strain sensor and the determination of that sensor wavelength shifts due to axial strains. We compared the results using the proposed time interval measurement with OSA measurements and found deviations within $\pm 200 \mathrm{pm}$ after processing data with a maximum peak search algorithm. Results like that were because of the large bandwidth FBG sensors used and their spectral shape and the sensitivity of the algorithm to amplitude fluctuations. However, improved results were obtained after applying a Gaussian curve fit to the detected traces, which yield an error within $22 \mathrm{pm}$. 


\section{CHAPTER 7}

\section{CONCLUSIONS}

In this research, the use of a silicon micro-ring resonator device as a wavelength interrogator was successfully demonstrated. This application opens up the possibility of embedding an interrogation system on a chip because of the size of the optical components. Compared to other FBG sensor detection solutions, using high Q narrow-band resonance micro-ring filters makes it possible to improve the measurement resolution of a wavelength shift detection system. Other FBG sensor detection methods relied on mechanically tuned filters, with this design the tuning mechanism is electro-thermally controlled.

To accomplish this study, a thermally tuned micro-ring resonator device was designed and tested. The device used in this research is a compact $(12 \mu m \times 12 \mu m)$ ring structure with $5 \mu m$ radius and a waveguide aspect ratio of 1.8. This design yielded a quasi-TE FSR of $\sim 18 \mathrm{~nm}$, a filter line-width (FWHM) between 0.13 to $0.19 \mathrm{~nm}$ and $\mathrm{Q}$ factors between 8000 to 10000 . In terms of the tuning response of this device, we demonstrated a tunable range of $3 \mathrm{~nm}$ at $5 \mathrm{~mA}$ current applied to a micro-heater. It is worth mentioning that the design study of this device was accomplished by developing a photonic toolkit based on the use of open-source software and models found in the literature. That decision had the advantage of integrating many software modules for optical device simulation together with numerical data analysis tools with the benefit of applying scripts to analyze design decisions from a set of engineering parameters.

An application of the use of the silicon micro-ring resonator as a wavelength interrogator was described in this research. A basic system model was contributed, which leads to the development of a system simulation. The simulation results helped us to understand the effect of micro-ring tuning on the shape of the spectrum traces in the scan- 
ning operation. Noise performance simulations showed that high $\mathrm{Q}$ filters with narrow bandwidth FBG sensors should yield detection resolutions less than $10 \mathrm{pm}$.

Experimental tests of the wavelength detection system were demonstrated. The center wavelength of a laser was detected in this system by measuring the time interval between peaks. This result served us as a calibration curve for sensor interrogation. The experimental oscilloscope traces obtained with this method were similar to simulated results using our simplified model.

Finally, we demonstrate the interrogation of a FBG fiber functioning as a strain sensor. Wavelength shift detection results with this method were close to the results measured by an OSA. The deviation of this method was close to $22 \mathrm{pm}$ compared to a linear fit taken from OSA measurements. Even that the FBG sensor itself had low spectral quality, by applying Gaussian curve fit on the data it was possible to improve the wavelength shift estimations compared to a simple maximum peak search algorithm. In general, although other SOI interrogators were implemented to process tilted FBG sensors [106], as far as this author's knowledge this is the fist time a wavelength shift interrogator was implemented and experimentally demonstrated using a tunable micro-ring resonator.

Implications of this study can be summarized in the following: a micro-ring filter device can be applied as a sensor wavelength interrogator with the advantage of its compact size, scalability and versatility. For example, besides FBG sensor interrogation, the system can be used to convert optical wavelength shifts into electrical signals coming from integrated photonic sensors measuring temperature [113] or protein detection/DNA analysis [19].

\subsection{Future Work}

The basics of this device's operating functions can be further improved and extended into new applications, especially in heterogeneous systems in a chip designs. The micro-ring 
resonator can be improved in terms of reducing internal losses and double-peak resonance signatures. One way to do that is by certain fabrication techniques, such as oxidation smoothing [103]. Also, improvements in the micro-heaters by geometrical optimizations can yield low power and fast thermo-optical responses, as shown in [114]. Additionally, in order to reduce dependence on external equipment, it is possible to introduce embedded Ge photo-detectors at the micro-ring output ports [76]. That will convert directly optical signals into electrical ones.

In terms of system improvements, the precise control of the thermal tuning may be needed. It was demonstrated in [115] that using the heater as a temperature sensor together with a temperature control circuit, then $80 \mathrm{mK}$ of thermal stability of the ring resonance can be achieved. Finally, because of silicon photonics compatibility with CMOS fabrication, the integration of the analog signal processing functions demonstrated in this system can be integrated. This feature will enable compact micro-opto-electronic systems on a chip with the potential to reach low cost integrated devices to perform high density sensor measurements. 


\section{BIBLIOGRAPHY}

[1] I. P. Kaminow. Optical integrated circuits: A personal perspective. Lightwave Technology, Journal of, 26(9):994-1004, 2008. 1

[2] M. Lipson. Guiding, modulating, and emitting light on silicon-challenges and opportunities. Lightwave Technology, Journal of, 23(12):4222-4238, 2005. 1, 3, 10,62

[3] X. Wang, J. A. Martinez, M. S. Nawrocka, and R. R. Panepucci. Compact thermally tunable silicon wavelength switch: Modeling and characterization. Photonics Technology Letters, IEEE, 20(11):936-938, 2008. 1, 20, 58, 64, 69, 90, 96, 99 , $100,105,118$

[4] Ng Han-Yong, M.R. Wang, Daqun Li, Xuan Wang, J. Martinez, R.R. Panepucci, and K. Pathak. 1x4 wavelength reconfigurable photonic switch using thermally tuned microring resonators fabricated on silicon substrate. Photonics Technology Letters, IEEE, 19(9):704-706, 2007. 1

[5] Xuan Wang, Tao Liu, Vilson R. de Almeida, and Roberto R. Panepucci. Onchip silicon photonic wavelength control of optical fiber lasers. Optics Express, 16(20):15671-15676, 2008. 2, 58, 81

[6] Katrien De Vos, Irene Bartolozzi, Etienne Schacht, Peter Bienstman, and Roel Baets. Silicon-on-Insulator microring resonator for sensitive and label-free biosensing. Optics Express, 15(12):7610-7615, June 2007. 2, 10

[7] Vilson R. Almeida, Carlos A. Barrios, Roberto R. Panepucci, and Michal Lipson. All-optical control of light on a silicon chip. Nature, 431(7012):1081-1084, October 2004. 2, 32, 62

[8] Magdalena S. Nawrocka, Tao Liu, Xuan Wang, and Roberto R. Panepucci. Tunable silicon microring resonator with wide free spectral range. Applied Physics Letters, 89(7):071110-3, 2006. 2, 20, 69, 88, 99

[9] Yurii Vlasov and Sharee McNab. Losses in single-mode silicon-on-insulator strip waveguides and bends. Optics Express, 12(8):1622-1631, April 2004. 2, 4, 16, 64

[10] Milos Popovic. Theory and Design of High-Index-Contrast Microphotonic Circuits. Ph.D. dissertation, Massachusetts Institute of Technology, February 2008. xi, 3, 58, 64, 66, 118 
[11] Vilson R. Almeida, Roberto R. Panepucci, and Michal Lipson. Nanotaper for compact mode conversion. Optics Letters, 28(15):1302-1304, 2003. 4

[12] Richard SOREF. The impact of silicon photonics. IEICE Trans Electron, E91C(2):129-130, February 2008. 4

[13] J. M Lopez-Higuera, L. Rodriguez Cobo, A. Quintela Incera, and A. Cobo. Fiber optic sensors in structural health monitoring. Journal of Lightwave Technology, 29(4):587-608, February 2011. 4, 34

[14] José Miguel López-Higuera. Optical Sensors. Ed. Universidad de Cantabria, 1998. 4

[15] K. O Hill and G. Meltz. Fiber bragg grating technology fundamentals and overview. Journal of Lightwave Technology, 15(8):1263 - 1276, 1997. 6, 36, $40,41,114$

[16] Alan D. Kersey. Fiber grating sensors. Journal of Lightwave Technology, 15(8):1442-1463, 1997. 6, 35, 36, 39, 40, 41, 42, 43, 44, 45, 46, 50, 97

[17] Biqiang Jiang, Jianlin Zhao, Chuan Qin, Zhao Huang, and Fan Fan. An optimized strain demodulation method based on dynamic double matched fiber bragg grating filtering. Optics and Lasers in Engineering, 49(3):415-418, March 2011. 6

[18] A. Mendez. Fiber bragg grating sensors: a market overview. Proceedings of SPIE, 6619(1):661905-661905-6, July 2007. 6, 34

[19] Mario Iodice, Luca De Stefano, Giuseppe Coppola, Vito Mocella, Ilaria Rea, Edoardo De Tommasi, Emanuele Orabona, and Ivo Rendina. Label-free biosensing by means of optical micro-ring resonator. Proceedings of SPIE, 7356:735603735603-11, 2009. 6, 122

[20] G. R. Vargas and R. R. Panepucci. Wavelength monitoring using a thermally tuned micro-ring resonator. In Numerical Simulation of Optoelectronic Devices (NUSOD), 2010 10th International Conference on, pages 83-84, Atlanta, GA, 2010. IEEE. 7, 58, 103

[21] Jose Mora, Jose Luis Cruz, Miguel V. Andres, and Ricardo Duchowicz. Simple High-Resolution wavelength monitor based on a fiber bragg grating. Applied Optics, 43(4):744-749, February 2004. 8, 45, 55, 56, 100 
[22] Xuan Wang and Roberto R. Panepucci. Multiplexed FBG interrogation system on a silicon chip. In Integrated Photonics and Nanophotonics Research and Applications (IPNRA). OSA, 2009. 10, 52, 73

[23] B.E. Little, S.T. Chu, H.A. Haus, J. Foresi, and J.-P. Laine. Microring resonator channel dropping filters. Lightwave Technology, Journal of, 15(6):998-1005, 1997. $10,22,23,25,27,32,97$

[24] Qianfan Xu, Sasikanth Manipatruni, Brad Schmidt, Jagat Shakya, and Michal Lipson. $12.5 \mathrm{gbit} / \mathrm{s}$ carrier-injection-based silicon micro-ring silicon modulators. $O p$ tics Express, 15(2):430-436, January 2007. 10

[25] B. Moslehi, J.W. Goodman, M. Tur, and H.J. Shaw. Fiber-optic lattice signal processing. Proceedings of the IEEE, 72(7):909-930, 1984. 10, 14

[26] A. Yariv. Universal relations for coupling of optical power between microresonators and dielectric waveguides. Electronics Letters, 36(4):321-322, 2000. 13

[27] G.T. Paloczi, J. Scheuer, and A. Yariv. Compact microring-based wavelengthselective inline optical reflector. Photonics Technology Letters, IEEE, 17(2):390392, 2005. 13

[28] G. Barbarossa, A.M. Matteo, and M.N. Armenise. Theoretical analysis of triplecoupler ring-based optical guided-wave resonator. Lightwave Technology, Journal of, 13(2):148-157, 1995. 14

[29] Andreas Vörckel, Mathias Mönster, Wolfgang Henschel, Peter Haring Bolivar, and Heinrich Kurz. Asymmetrically coupled silicon-on-insulator microring resonators for compact add-drop multiplexers. IEEE Photonics Technology Letters, 15(7):921-923, 2003. 16, 18, 22

[30] O. Schwelb. Transmission, group delay, and dispersion in single-ring optical resonators and add/drop filters-a tutorial overview. Lightwave Technology, Journal of, 22(5):1380-1394, 2004. 16, 22

[31] Shijun Xiao, Maroof H. Khan, Hao Shen, and Minghao Qi. Compact silicon microring resonators with ultra-low propagation loss in the c band. Optics Express, 15(22):14467-14475, October 2007. 16, 74, 80

[32] Shijun Xiao, Maroof H. Khan, Hao Shen, and Minghao Qi. Modeling and measurement of losses in silicon-on-insulator resonators and bends. Optics Express, 15(17):10553-10561, 2007. 20, 29, 30, 31, 32, 74, 88 
[33] Trevor M. Benson, Svetlana V. Boriskina, Phillip Sewell, Ana Vukovic, Stephen C. Greedy, and Alexander I. Nosich. Micro-optical resonators for microlasers and integrated optoelectronics: recent advances and future challenges. arXiv:physics/0607239, July 2006. Frontiers of Planar Lightwave Circuit Technology: Design, Simulation and Fabrication (S. Janz, J. Ctyroky and S. Tanev eds.), pp. 39-70, Springer 2005. 20

[34] A.M. Prabhu, A. Tsay, Zhanghua Han, and Vien Van. Ultracompact SOI microring Add-Drop filter with wide bandwidth and wide FSR. Photonics Technology Letters, IEEE, 21(10):651-653, 2009. 21, 69

[35] Jan Niehusmann, Andreas Vörckel, Peter Haring Bolivar, Thorsten Wahlbrink, Wolfgang Henschel, and Heinrich Kurz. Ultrahigh-quality-factor silicon-oninsulator microring resonator. Optics Letters, 29(24):2861-2863, December 2004. $21,74,88$

[36] Amnon Yariv and Pochi Yeh. Photonics. Oxford University Press, 2006. 21, 30

[37] Hermann A. Haus. Waves and fields in optoelectronics. Prentice-Hall, 1984. 23, $24,27,75$

[38] J.D. Joannopoulos. Photonic crystals: molding the flow of light. Princeton University Press, 2008. 23, 24, 65

[39] R Dekker, N Usechak, M Först, and A Driessen. Ultrafast nonlinear all-optical processes in silicon-on-insulator waveguides. Journal of Physics D: Applied Physics, 40(14):R249-R271, July 2007. 32

[40] Ph. M. Nellen, P. Mauron, A. Frank, U. Sennhauser, K. Bohnert, P. Pequignot, P. Bodor, and H. Brandle. Reliability of fiber bragg grating based sensors for downhole applications. Sensors and Actuators A: Physical, 103(3):364-376, February 2003. 34

[41] Roberto Montanini. Wavelength-encoded optical psychrometer for relative humidity measurement. Review of Scientific Instruments, 78(2):025103, 2007. 34

[42] L. Dziuda, G. Fusiek, P. Niewczas, G.M. Burt, and J.R. McDonald. Laboratory evaluation of the hybrid fiber-optic current sensor. Sensors and Actuators A: Physical, 136(1):184-190, May 2007. 34 
[43] Serge M. Melle, Kexing Liu, and Raymond M. Measures. Practical fiber-optic bragg grating strain gauge system. Applied Optics, 32(19):3601-3609, July 1993. $35,42,44,97$

[44] Youlong Yu and Hongxia Zhao. A novel demodulation scheme for fiber bragg grating sensor system. Photonics Technology Letters, IEEE, 17(1):166-168, 2005. 35

[45] Andreas Othonos. Fiber bragg gratings. Review of Scientific Instruments, 68:4309, 1997. 35, 38, 40, 41

[46] C.L. Barbosa, H.T. Hattori, O. Lisboa, R.C. Rabelo, and Rogerio Moreira Cazo. Fabrication and characterization of Bragg gratings through phase mask technique. Revista do Inatel - Sao Paulo, 3:22-26, 2000. 36

[47] A. Agarwal, T. McKechnie, S. Starrett, and M. M. Opeka. Near net shape forming of hafnium-based ceramic components: Synthesis and characterization. In Elevated Temperature Coatings-4, TMS 2001 Annual Meeting, pages 301-316, New Orleans, February 2001. 36

[48] A. B. Lobo Ribeiro, L. A. Ferreira, J. L. Santos, and D. A. Jackson. Analysis of the reflective-matched fiber bragg grating sensing interrogation scheme. Applied Optics, 36(4):934-939, February 1997. 38, 39, 47

[49] Allan C. L. Wong. Fibre-optic Sensing Technology and Applications in Civil Engineering. Ph.D. dissertation, The University of New South Wales, Sidney, Australia, 2007. 41

[50] Xiaochun Li. Embedded Sensors in Layered Manufacturing. Ph.D. dissertation, Stanford University, Palo Alto, CA, 2001. 41, 114

[51] O Frazão and J L Santos. Simultaneous measurement of strain and temperature using a bragg grating structure written in germanosilicate fibres. Journal of Optics A: Pure and Applied Optics, 6:553-556, June 2004. 41

[52] Horst Czichos, Tetsuya Saito, Leslie R. Smith, and Leslie Smith. Springer handbook of materials measurement methods, volume 978. Springer, November 2006. 42

[53] Qian Wang, Gerald Farrell, Pengfei Wang, Ginu Rajan, and Thomas Freir. Design of integrated wavelength monitor based on a y-branch with an s-bend waveguide. Sensors and Actuators A: Physical, 134(2):405-409, March 2007. 42, 44, 48, 97 
[54] O. Schmidt, P. Kiesel, S. Mohta, and N.M. Johnson. Resolving pm wavelength shifts in optical sensing. Applied Physics B: Lasers and Optics, 86(4):593-600, March 2007. 42

[55] A.S. Paterno, V. de Oliveira, T.S. Figueredo, and H.J. Kalinowski. Multiplexed fiber bragg grating interrogation system using a modulated fiber bragg grating and the Tunable-Filter method. Sensors Journal, IEEE, 6(6):1662-1668, 2006. 43, 44, $45,47,49,50,51,52,59$

[56] Michael L. Dennis, Martin A. Putnam, Jin U. Kang, Tsung-Ein Tsai, Irl N. Duling III, and E. Joseph Friebele. Grating sensor array demodulation by use of a passively mode-locked fiber laser. Opt. Lett., 22(17):1362-1364, 1997. 43

[57] M. Suleiman, H.C. Seat, and T. Bosch. Interrogation of fiber bragg grating dynamic strain sensors by Self-Mixing interferometry. Sensors Journal, IEEE, 8(7):13171323, 2008. 43, 44

[58] M. G. Xu, H. Geiger, and J.P. Dakin. Modeling and performance analysis of a fiber bragg grating interrogation system using an acousto-optic tunable filter. Lightwave Technology, Journal of, 14(3):391-396, 1996. 45, 50, 53, 54

[59] A. D. Kersey, T. A. Berkoff, and W. W. Morey. Multiplexed fiber bragg grating strain-sensor system with a fiber fabry - perot wavelength filter. Optics Letters, 18(16):1370, 1993. 45, 46, 50

[60] Rongqing Hui and Maurice S. (Maurice Stephen) O'Sullivan. Fiber Optic Measurement Techniques. Academic Press, 2009. 45

[61] T Allsop, T Earthrowl, R Reeves, D J Webb, and I Bennion. The interrogation and multiplexing of long period grating curvature sensors using a bragg grating based, derivative spectroscopy technique. Measurement Science and Technology, 15(1):44-48, January 2004. 46

[62] Pawel Kluczynski, Jorgen Gustafsson, Asa M. Lindberg, and Ove Axner. Wavelength modulation absorption spectrometry - an extensive scrutiny of the generation of signals. Spectrochimica Acta Part B: Atomic Spectroscopy, 56(8):12771354, August 2001. 46, 49

[63] Luis Alberto Ferreira, Envangelos Vasilios Diatzikis, Jose Luis Santos, and Faramarz Farahi. Demodulation of fiber bragg grating sensors based on dynamic tuning of a multimode laser diode. Applied Optics, 38(22):4751-4759, 1999. 47, 48, 50, 51,53 
[64] Marcel W. Pruessner, Todd H. Stievater, Mike S. Ferraro, and William S. Rabinovich. Thermo-optic tuning and switching in SOI waveguide Fabry-Perot microcavities. Optics Express, 15(12):7557-7563, June 2007. 48

[65] Milton Abramowitz. Handbook of Mathematical Functions with Formulas, Graphs, and Mathematical Tables. Wiley, 1972. 49

[66] C. Caucheteur, K. Chah, F. Lhomme, M. Blondel, and P. Megret. Autocorrelation demodulation technique for fiber bragg grating sensor. Photonics Technology Letters, IEEE, 16(10):2320-2322, 2004. 50, 116

[67] Hyun-Wook Lee, Hyoung-Jun Park, June-Ho Lee, and Minho Song. Accuracy improvement in peak positioning of spectrally distorted fiber bragg grating sensors by gaussian curve fitting. Applied Optics, 46(12):2205-2208, April 2007. 50, 116, 118

[68] David J. F. Cooper and Peter W. E. Smith. Limits in wavelength measurement of optical signals. Journal of the Optical Society of America B, 21(5):908-913, May 2004. 50

[69] Xuan Wang, German R Vargas, and Roberto Panepucci. FBG interrogation system on a silicon chip. In Latin America Optics and Photonics Conference 2010, Recife, Brazil, September 2010. OSA. 52, 119

[70] W.R. Allan, Z.W. Graham, J.R. Zayas, D.P. Roach, and D.A. Horsley. Multiplexed fiber bragg grating interrogation system using a microelectromechanical FabryPerot tunable filter. Sensors Journal, IEEE, 9(8):936-943, 2009. 54

[71] Kun Liu, Wen-cai Jing, Tie-gen Liu, Da-gong Jia, Hong-xia Zhang, and Yi-mo Zhang. Design and nonlinearity compensation of Fabry-Perot type tunable optical filters for dynamic strain sensing systems. Optoelectronics Letters, 4(4):248-252, July 2008. 54

[72] G.R. Vargas and R.R. Panepucci. Wavelength to time interval demodulation employing a tunable micro-ring. In Microwave \& Optoelectronics Conference (IMOC), 2011 SBMO/IEEE MTT-S International, pages 837 -841, Natal, Brazil, November 2011. IEEE. xiii, 58, 99, 100, 101, 102, 103

[73] A. Kerrouche, W.J.O. Boyle, T. Sun, and K.T.V. Grattan. Design and in-the-field performance evaluation of compact FBG sensor system for structural health monitoring applications. Sensors and Actuators A: Physical, 151(2):107-112, April 2009. 59 
[74] Seth W. Lloyd, Jason A. Newman, Daniel R. Wilding, Richard H. Selfridge, and Stephen M. Schultz. Compact optical fiber sensor smart node. Review of Scientific Instruments, 78(3):035108, 2007. 59

[75] Safa O. Kasap. Optoelectronics and photonics. Prentice Hall, February 2001. 60

[76] L. Clavelier, C. Deguet, C. Le Royer, B. Vincent, J.-F. Damlencourt, J.-M. Hartmann, O. Kermarrec, T. Signamarcheix, B. Depuydt, A. Theuwis, C. Quaeyhaegens, N. Cherkashin, Y. Morand, P. Rivallin, C. Tabone, S. Lagrasta, Y. Campidelli, S. Descombes, L. Sanchez, T. Akastu, A. Rigny, D. Bensahel, T. Billon, N. Kernevez, and S. Deleonibus. Review of some critical aspects of ge and GeOI substrates. In ECS Trans., volume 3 of ECS Transactions, pages 789-805, Cancun, Mexico, 2006. Electrochemical Society Inc. 60, 123

[77] Newport. Estimating minimum power for a power meter and detector system, 2004. 61

[78] C. Angulo Barrios, V.R. Almeida, R.R. Panepucci, B.S. Schmidt, and M. Lipson. Compact silicon tunable Fabry-Perot resonator with low power consumption. IEEE Photonics Technology Letters, 16(2):506-508, 2004. 62

[79] INTEL. Intel milestone confirms light beams can replace electronic signals for future computers. http://www.intel.com/pressroom/archive/releases/2010/20100727comp_sm.htm. 62

[80] Luxtera. Luxtera I luxtera and STMicroelectronics to enable high-volume silicon photonics solutions. http://www.luxtera.com/20120301254/luxtera-andstmicroelectronics-to-enable-high-volume-silicon-photonics-solutions.html. 62

[81] B. Thomas Smith, D. Feng, H. Lei, D. Zheng, J. Fong, and M. Asghari. Fundamentals of silicon photonic devices. 62

[82] Caliopa. Caliopa - shaping the future of optical transceivers. http://caliopa.be/news-and-events/article/ugent-and-imec-launch-siliconphotonics-spin-off-caliopa. 62

[83] Otto Schwelb and Istvan Frigyes. Vernier operation of series-coupled optical microring resonator filters. Microwave and Optical Technology Letters, 39(4):257261, 2003. 62 
[84] William S. Fegadolli, German Vargas, Xuan Wang, Felipe Valini, Luis A. M. Barea, Jose E. B. Oliveira, Newton Frateschi, Axel Scherer, Vilson R. Almeida, and Roberto R. Panepucci. Reconfigurable silicon thermo-optical ring resonator switch based on vernier effect control. Optics Express, 20(13):14722, June 2012. 63,118

[85] M.A. Popovic, T. Barwicz, E.P. Ippen, and F.X. Kartner. Global design rules for silicon microphotonic waveguides: Sensitivity, polarization and resonance tunability. In Lasers and Electro-Optics, 2006 and 2006 Quantum Electronics and Laser Science Conference. CLEO/QELS 2006. Conference on, pages 1-2. OSA, 2006. 63

[86] N. Rouger, L. Chrostowski, and R. Vafaei. Temperature effects on Silicon-onInsulator (SOI) racetrack resonators: A coupled analytic and 2-D finite difference approach. Lightwave Technology, Journal of, 28(9):1380-1391, 2010. 64, 68, 69, $70,71,74,97$

[87] Steven G. Johnson and J. D. Joannopoulos. Block-iterative frequency-domain methods for maxwell's equations in a planewave basis. Opt. Express, 8(3):173190, 2001. 65

[88] Eric Dulkeith, Fengnian Xia, Laurent Schares, William M. J Green, and Yurii A. Vlasov. Group index and group velocity dispersion in silicon-on-insulator photonic wires. Optics Express, 14(9):3853-3863, May 2006. 65

[89] Soon T. Lim, Ching E. Png, Eng A. Ong, and Yong L. Ang. Single mode, polarization-independent submicron silicon waveguides based on geometrical adjustments. Optics Express, 15(18):11061-11072, 2007. 65

[90] Rsoft Design Group. Beamprop 7.0 user's guide, 2006. 66

[91] Arman B. Fallahkhair, Kai S. Li, and Thomas E. Murphy. Vector finite difference modesolver for anisotropic dielectric waveguides. Journal of Lightwave Technology, 26(11):1423-1431, June 2008. 66

[92] G. van Rossum and F. L. Drake. Python reference manual, 2006. 67, 103

[93] Travis E. Oliphant. Guide to NumPy. Brigham Young University, Provo, UT, March 2006. 67, 103

[94] Eric Jones, Travis Oliphant, and Peterson. SciPy: open source scientific tools for Python, 2001. 67, 103, 116 
[95] M. K. Chin and S. T. Ho. Design and modeling of Waveguide-Coupled SingleMode microring resonators. Journal of Lightwave Technology, 16(8):1433, 1998. 70

[96] S.C. Hagness, D. Rafizadeh, S.T. Ho, and A. Taflove. FDTD microcavity simulations: design and experimental realization of waveguide-coupled single-mode ring and whispering-gallery-mode disk resonators. Lightwave Technology, Journal of, 15(11):2154-2165, 1997. 75, 76, 77

[97] X. Zhang, W. Hong, and D. Huang. Simple method for spectral response simulation of micro-ring resonators by combining transfer matrix method with FDTD method. Electronics Letters, 42(19):1095-1096, 2006. 75

[98] Fujii Masafumi. Efficient High-Spatial-Order FDTD analysis of 3D optical ring resonator filters. In The 19th Annual Review of Progress in Applied Computational Electromagnetics, pages 739-744, Monterey, CA, 2003. 75

[99] Zhixuan Xia, Yao Chen, and Zhiping Zhou. Dual waveguide coupled microring resonator sensor based on intensity detection. Quantum Electronics, IEEE Journal of, 44(1):100-107, 2008. 75

[100] Zhechao Wang, Daoxin Dai, and Sailing He. Polarization-Insensitive ultrasmall microring resonator design based on optimized si sandwich nanowires. Photonics Technology Letters, IEEE, 19(20):1580-1582, 2007. 75

[101] Ardavan F. Oskooi, David Roundy, Mihai Ibanescu, Peter Bermel, J. D. Joannopoulos, and Steven G. Johnson. MEEP: a flexible free-software package for electromagnetic simulations by the FDTD method. Computer Physics Communications, 181:687-702, January 2010. 75, 77

[102] Allen Taflove and Susan C. Hagness. Computational electrodynamics: the finitedifference time-domain method. Artech House, June 2005. 75, 77

[103] Kevin K. Lee, Desmond R. Lim, Lionel C. Kimerling, Jangho Shin, and Franco Cerrina. Fabrication of ultralow-loss $\mathrm{Si} / \mathrm{SiO} 2$ waveguides by roughness reduction. Optics Letters, 26(23):1888-1890, December 2001. 92, 123

[104] Gaozhi Xiao, N. Mrad, Fang Wu, Zhiyi Zhang, and Fengguo Sun. Miniaturized optical fiber sensor interrogation system employing echelle diffractive gratings demultiplexer for potential aerospace applications. Sensors Journal, IEEE, 8(7):1202-1207, 2008. 97 
[105] Nezih Mrad, Gaozhi Xiao, Honglei Guo, Wolfgang Ecke, Kara J. Peters, and Norbert G. Meyendorf. Micro-size optical fibre strain interrogation system. volume 6933, pages 693304-6, San Diego, California, USA, March 2008. SPIE. 97

[106] Pavel Cheben, Edith Post, Siegfried Janz, Jacques Albert, Albane Laronche, Jens H. Schmid, Dan-Xia Xu, Boris Lamontagne, Jean Lapointe, Andre Delage, and Adam Densmore. Tilted fiber bragg grating sensor interrogation system using a high-resolution silicon-on-insulator arrayed waveguide grating. Optics Letters, 33(22):2647-2649, November 2008. 97, 122

[107] Lucas Negri, Ademir Nied, Hypolito Kalinowski, and Aleksander Paterno. Benchmark for peak detection algorithms in fiber bragg grating interrogation and a new neural network for its performance improvement. Sensors, 11(4):3466-3482, March 2011. 118

[108] Shellee D Dyer, Paul A Williams, R. Joseph Espejo, Jonathan D Kofler, and Shelley M Etzel. Fundamental limits in fiber bragg grating peak wavelength measurements. In Proceedings of SPIE, volume 5855 PART I, pages 88 - 93, Bruges,Belgium, 2005. 118

[109] P. Prabhathan, Zhang Jing, V.M. Murukeshan, Zhang Huijuan, and Chen Shiyi. Discrete and fine wavelength tunable thermo-optic WSS for low power consumption C+L band tunability. Photonics Technology Letters, IEEE, 24(2):152 -154, January 2012. 118

[110] Zhixuan Xia, Ali Asghar Eftekhar, Mohammad Soltani, Babak Momeni, Qing Li, Maysamreza Chamanzar, Siva Yegnanarayanan, and Ali Adibi. High resolution onchip spectroscopy based on miniaturized microdonut resonators. Optics Express, 19(13):12356-12364, June 2011. 119

[111] Mohammad Soltani, Qing Li, Siva Yegnanarayanan, and Ali Adibi. Toward ultimate miniaturization of high q silicon traveling-wave microresonators. Optics Express, 18(19):19541-19557, September 2010. 119

[112] Ciyuan Qiu, Jie Shu, Zheng Li, Xuezhi Zhang, and Qianfan Xu. Wavelength tracking with thermally controlled silicon resonators. Opt. Express, 19(6):5143-5148, March 2011. 119

[113] Gun-Duk Kim, Hak-Soon Lee, Chang-Hyun Park, Sang-Shin Lee, Boo Tak Lim, Hee Kyoung Bae, and Wan-Gyu Lee. Silicon photonic temperature sensor employing a ring resonator manufactured using a standard CMOS process. Optics Express, 18(21):22215-22221, October 2010. 122 
[114] A. H. Atabaki, E. Shah Hosseini, A. A. Eftekhar, S. Yegnanarayanan, and A. Adibi. Optimization of metallic microheaters for high-speed reconfigurable silicon photonics. Optics Express, 18(17):18312-18323, 2010. 123

[115] R. Amatya, C.W. Holzwarth, H.I. Smith, and R.J. Ram. Precision tunable silicon compatible microring filters. Photonics Technology Letters, IEEE, 20(20):17391741, 2008. 123 
VITA

\section{GERMAN R VARGAS}

Born, Guayaquil, Ecuador

1997

1997-1999

B.Sc. Electrical Engineering

Escuela Superior Politecnica del Litoral

Guayaquil, Ecuador

Applications Engineer

Gruein Cia. Ltda.

Guayaquil, Ecuador

2001

M.Sc. Electrical Engineering

Arizona State University

Tempe, Arizona

$2003-2007$

Assistant Professor

Escuela Superior Politecnica del Litoral

Guayaquil, Ecuador

$2007-2009$

Teaching Assistant

Department of Electrical and Computer Engineering

Florida International University

Miami, FL

\section{PUBLICATIONS AND PRESENTATIONS}

William S. Fegadolli, German Vargas, Xuan Wang, Felipe Valini, Luis A. M. Barea, Jose E. B. Oliveira, Newton Frateschi, Axel Scherer, Vilson R. Almeida, and Roberto R. Panepucci. Reconfigurable silicon thermo-optical ring resonator switch based on vernier effect control. Optics Express, 20(13):14722, June 2012.

G.R. Vargas and R. R. Panepucci. "Wavelength to Time Interval Demodulation Employing a Tunable Micro-ring Resonator". in International Microwave and Optoelectronics Conference (IMOC), (SBMO/IEEE MTT-S, 2011)

G.R. Vargas and R. R. Panepucci. "Wavelength monitoring using a thermally tuned microring resonator". in 10th International Conference of Numerical Simulation of Optoelectronic Devices (NUSOD), (IEEE, 2010), pp 83-84. 
X. Wang, G. R. Vargas, and R. R. Panepucci, "FBG Interrogation System on a Silicon Chip," in Latin America Optics and Photonics Conference, OSA Technical Digest (CD) (Optical Society of America, 2010), paper ThF3.

Khanal, Chooda; Vargas, Garman; Balani, Kantesh; Keshri, Anup; Barbosa, Carmen; Agarwal, Arvind and Panepucci, Roberto, "Metal embedded Fiber Brag Grating Sensors," in American Physics Society March Meeting (APS, 2009). 